University of Louisville

ThinkIR: The University of Louisville's Institutional Repository

Electronic Theses and Dissertations

$5-2005$

\title{
Toward autonomic distributed data mining using intelligent web services.
}

Padmanabhan Ramaswamy 1976-

University of Louisville

Follow this and additional works at: https://ir.library.louisville.edu/etd

\section{Recommended Citation}

Ramaswamy, Padmanabhan 1976-, "Toward autonomic distributed data mining using intelligent web services." (2005). Electronic Theses and Dissertations. Paper 1177.

https://doi.org/10.18297/etd/1177

This Master's Thesis is brought to you for free and open access by ThinkIR: The University of Louisville's Institutional Repository. It has been accepted for inclusion in Electronic Theses and Dissertations by an authorized administrator of ThinkIR: The University of Louisville's Institutional Repository. This title appears here courtesy of the author, who has retained all other copyrights. For more information, please contact thinkir@louisville.edu. 


\title{
Toward Autonomic Distributed Data Mining USING INTELLIGENT WEB SERVICES
}

\author{
By
}

\section{Padmanabhan Ramaswamy}

BS., Kerala University, 1998

\author{
A Thesis \\ Submitted to the Faculty of the \\ Speed scientific School \\ University of Louisville \\ As Partial Fulfillment of the Requirements \\ For the Professional Degree
}

Master of Science

Computer Engineering and Computer Science Department

University of Louisville

Louisville KY

May 2005 


\title{
TOWARD AUTONOMIC DISTRIBUTED DATA MINING USING INTELLIGENT WEB SERVICES
}

\author{
By \\ Padmanabhan Ramaswamy \\ B.S., Kerala University, 1998
}

A Thesis approved on

April 29, 2005

By the following Reading and Examination Committee

Dr. Anup Kumar, Co-Advisor (CECS)

Dr. Mehmed Kantardzic, Co-Advisor (CECS)

Dr. Adel Elmaghraby, Member (CECS)

Dr. Julius Wong, Member (ME) 


\section{ACKNOWLEDGEMENTS}

I would like to gratefully acknowledge the enthusiastic supervision of Dr. Anup Kumar who provided me with insights that guided me and challenged my thinking, substantially improving the finished product. I thank Dr. Mehmed Kantardzic for providing me with on-going support throughout the project and for the technical discussions we had on the Distributed Data Mining model. I feel a lasting gratitude toward the faculty of Speed School who were excellent teachers and advisors.

I would also like to thank all my friends and family, wherever they are, particularly my Mum and Dad.

The study was in part supported by Kentucky Science \& Engineering Foundation grant KSEF-323-RDE-003 and NSF grant INF 7-001-006. 


\section{AbSTRACT \\ Toward Autonomic Distributed Data Mining using \\ INTELLIGENT WEB SERVICES}

\section{Padmanabhan Ramaswamy}

May 1, 2005

This study defines a new approach for building a Web Services based infrastructure for distributed data mining applications. The proposed architecture provides a roadmap for "autonomic" functionality of the infrastructure hiding the complexity of implementation details and enabling the user with a new level of usability in data mining process. Web Services based infrastructure delivers all required data mining activities in a utility-like fashion enabling heterogeneous components to be incorporated in a unified manner. Moreover, this structure allows the implementation of data mining algorithms for processing data on more than one source in a distributed manner. The purpose of this study is to present a simple, but efficient methodology for determining when data distributed at several sites can be centralized and analyzed as data from the same theoretical distribution. This analysis also answers when and how the semantics of the sites is influenced by distribution in data. This hierarchical framework with advanced and core Web Services improves the current data mining capability significantly in terms of performance, scalability, efficiency, transparency of resources, and incremental extensibility. 


\section{TABLE OF CONTENTS}

Acknowledgements....................................................

Abstract............................................................... iv

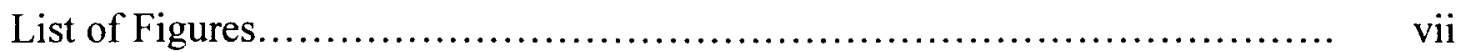

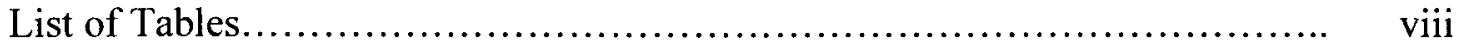

\section{ChaPTer 1}

Introduction.......................................................... 1

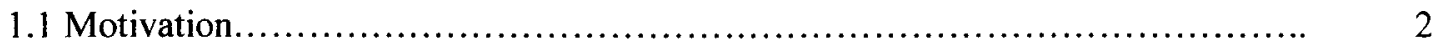

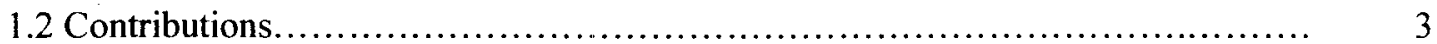

1.3 Thesis Organization..................................................... 5

\section{ChaPTER 2}

Related Research...................................................... 6

2.1 Existing Systems............................................................... 7

2.2 Distributed Data Mining.................................................... 10

2.3 Web Services........................................................ 13

2.4 SOAP Based Web Services............................................... 15

\section{Chapter 3}

Web Based Distributed Data Mining Framework............................ 16

3.1 Service Oriented Approach................................................ 17

3.2 Execution Framework ...................................................... 18

3.3 Detailed Architecture..................................................... 19 


\section{Chapter 4}

Implementation Framework.............................................. 22

4.1 Detailed System Architecture ................................................... 22

4.2 Implementation of the Services Framework ......................................... 27

4.2.1 Data Services................................................................ 28

4.2.2 Algorithm Services...................................................... $\quad 30$

4.2.3 Centralized Services...................................................... 32

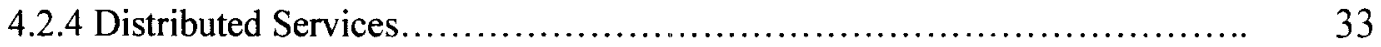

4.3 Selecting Dataset and Algorithm Service information........................... 34

4.3.1 Execution - Centralized Approach......................................... 35

4.3.2 Execution - Distributed Approach.......................................... $\quad 36$

4.3.3 Service Cost Calculation.................................................. 37

\section{Chapter 5}

Mining Algorithms as Web Services................................................ 40

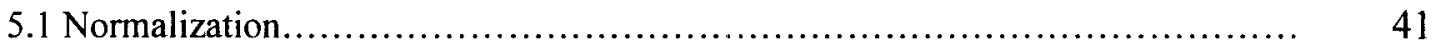

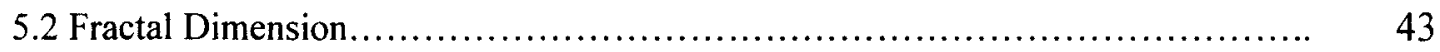

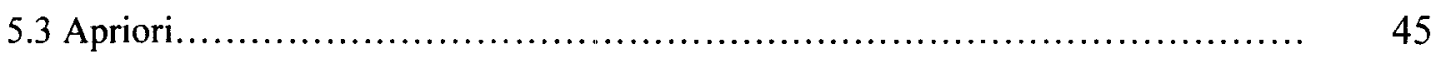

\section{ChAPTER 6}

Results and Discussion......................................................... 48

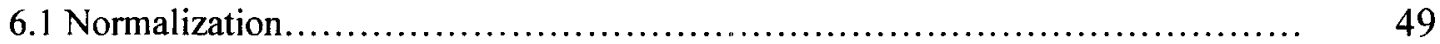

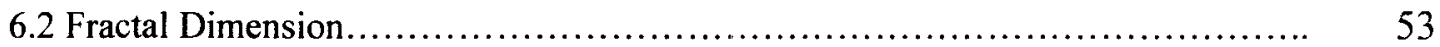

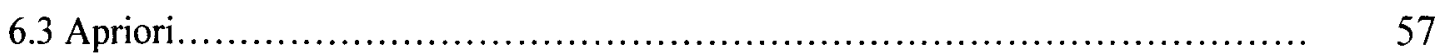

\section{ChaPTER 7}

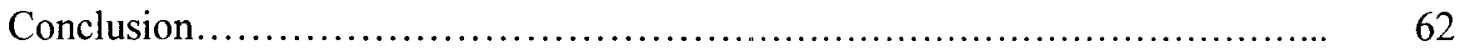

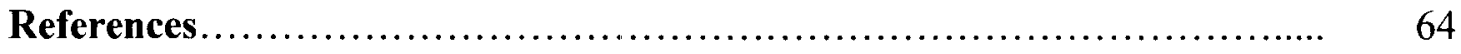

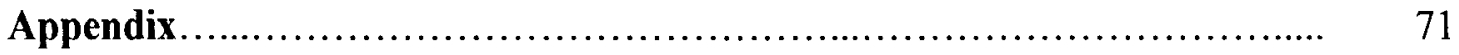

Curriculum Vitae........................................................... 100 


\section{LIST OF FIGURES}

\section{CHAPTER 2}

2.1 Hierarchical structure of a (Distributed) Data Mining Model..................... 12

2.2 Examples of paths in hierarchical structure of a data mining process............... 13

\section{Chapter 3}

3.1 Execution Framework....................................................... 19

3.2 Architecture for hierarchical Web Component service execution................... 20

\section{CHAPTER 4}

4.1 The registration process where the service providers register their services.......... 23

4.2 Structure of distributed data mining approach for distributed data.................. 25

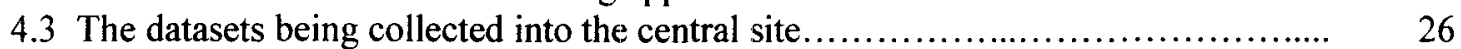

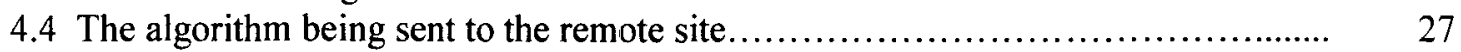

4.5a Step 1 of the Data Service registration process................................. 28

$4.5 b$ Step 2 of the Data Service registration process.................................. 29

$4.5 \mathrm{c}$ Registry response for the Data Service registration process....................... 29

4.6a Step 1 of the Algorithm Service registration process.......................... 30

4.6b Step 2 of the Algorithm Service registration process.......................... 31

$4.6 \mathrm{c}$ Registry response for the Algorithm Service registration process................ 31

4.8 The client requests to view service information from the registry................. 34

4.9 Client interaction in Centralized Approach................................... 35

4.10 Client interaction in Distributed Approach................................ 37

4.11 Data field selection and cost information................................... 38

4.12 Algorithm cost information................................................ 39

4.13 Final cost information................................................... 39

\section{CHAPTER 6}

6.1 Timing Results the Normalization algorithm in a LAN................................... 51

6.2 Timing Results the Normalization algorithm in a WAN............................... 52

6.3 Timing Results the Fractal Dimension algorithm in a LAN............................... 55

6.4 Timing Results the Fractal Dimension algorithm in a WAN........................ 56

6.5 Timing Results for the Centralized approach................................ 59

6.6 Timing Results for the Distributed approach................................. 60

6.7 Percentage improvement.................................................... 61 


\section{LIST OF TABLES}

\section{ChaPTER 3}

3.1 Functionality of the core components in the proposed framework given in

Figure 3.2.....

\section{Chapter 4}

4.1 Registration information for a service.

\section{Chapter 6}

6.1 Execution Times for the Normalization algorithm in a Local Area Network (Fractal Dimension Algorithm).

6.2 Execution Times for the normalization algorithm in a Wide Area Network (Fractal Dimension Algorithm).

6.3 Execution Times for the Fractal Dimension algorithm in a Local Area Network (Fractal Dimension Algorithm)

6.4 Execution Times for the Fractal Dimension algorithm in a Wide Area Network (Fractal Dimension Algorithm).

6.5 Execution Times in Milliseconds for the Centralized approach (Apriori Algorithm).

6.6 Execution Times in Milliseconds for the Distributed approach (Apriori Algorithm).

6.7 Percentage Improvement of Distributed approach Vs Centralized approach (Apriori Algorithm) 


\section{INTRODUCTION}

Information technology and data mining technology have lingered too long in an era of over-specialization in which integration is just another specialty. We have made a tremendous progress in almost every aspect of computing through these specializations in computer science and engineering. Some components are smaller, others are faster, cheaper, easily connectable, more precise, and have more capacity. How do we deal with the complexity of the entire environment generated by all that "smaller/faster/cheaper" focus, where complexity is expressed by heterogeneity and large interconnectivity of powerful software, hardware, and data components? Though this question is applicable to different IT disciplines, our concentration is on solutions for the data mining domain. Data mining technology is a typical example where research has made a lot of progress in specific algorithms and tools, but there are not enough results on their integration and simplified use in complex, distributed Internet based environments $[8,11]$.

Moreover, the assumptions and approaches used for static and centralized data mining processes are not any more valid for distributed environment [9]. Distributed data mining (DDM) refers to the mining of distributed data sets using distributed computational resources. The physical distribution of data through different sites in general corresponds to a semantic distribution, i.e., the location of data may have a 
meaning that needs to be explicitly addressed in a distributed mining process [13]. Data mining algorithms should take place at a local level to discover characteristics of the local site, and at a global level where local data mining results may be combined to find global findings or compare the local semantics. In distributed data mining strategy, it is necessary to provide the means of learning how to analyze, combine, and integrate a number of separately learned dynamic local models. Real-time data mining analysis are highly desirable in many distributed applications on the Internet to update models when new events are detected $[3,4,6]$. Easy distribution of models in a networked environment is essential for maintaining up to date detection capabilities.

Furthermore, the simplified implementation of algorithms and methods developed for centralized data mining applications could make significant influence on a reduced quality of data mining results [14] in the distributed environment. Therefore, it is necessary to reevaluate all data mining phases and all algorithms in the context of distributed resources applied for real-time data mining. This research shows the need for new phases or updated phases in the data mining process $[11,15]$.

\subsection{Motivation}

There are many reasons to investigate the use of web services in distributed data mining systems. Today most of the data mining is done in three-tier or four-tier system. In a three-tier system client sends the request to server, the server then runs mining algorithms on its local data. In a four-tier system, the server talks to another remote server, the remote server analyzes its local data. In both the systems, mining algorithms 
and the data are tied to the server making it a closed system. An extendable and open distributed data mining system can be built by separating the algorithm and data.

Using web services the interaction between algorithm and data can be made dynamic. Also, the algorithm can dynamically locate and analyze the data making it a much more robust system. The main motivation for this thesis involves the realization that using web services a dynamic distributed data mining system can be built.

\subsection{CONTRibutions}

The Web Services oriented approach proposed in this project is an attempt to build an infrastructure for DDM that will allow the integration of distributed, heterogeneous environment and complex interconnectivity. This approach will overcome the complexity of DDM applications and the limitations of existing infrastructures using Web Services technology $[7,8]$. The proposed architecture will allow users to concentrate on what they want to accomplish rather than figuring how to solve all the technical details in tuning computing system for executing distributed data mining models. The Web Services based infrastructure delivers required data mining activities in a utility-like fashion enabling heterogeneous components to cooperate in a unified manner. The exchange and integration of data and tasks (tools, libraries, device drivers, middleware, etc.) will be implemented through open standards defining the identification of components, their communication protocol and negotiation protocol among them. The key characteristics of the proposed data mining framework are: 
Dynamic: Dynamic discovery and use of data sets and data mining algorithms over the Internet based on user defined high-level specifications, and integration into distributed data mining process is one of the essential characteristics of the framework. It will tap the available resources, even negotiate their use, adapting to the conditions in the environment and requirements of the mining process.

Scalable: The Web Services based technologies used in this framework will allow integration of new data sources, algorithms, and tools available on the internet. In addition, clients from any platform will be able to use these new services. The framework will find a way to best interact with other neighboring systems.

Transparent: The complexity of data mining should be transparent to the users of the. In the proposed framework user focuses on what he/she wants to achieve with data mining and not on how to perform data mining. Therefore, the proposed framework hides the complexity of data mining operation built over heterogeneous and distributed Internet.

Extensible: The framework allows incremental and dynamic extensions of data sets and applied algorithms used in a distributed data mining process. Interactive configuration and dynamic reconfiguration of specified services under varying conditions, and adjustments of configurations to best handle changing web environment will be an important feature of our system. 


\subsection{Thesis Organization}

The thesis is organized in seven chapters. Chapter 2 summarizes the related research and a model for Distributed Data Mining. Chapter 3 discusses the proposed service oriented architecture. Registration and implementation details for various distributed datasets and data mining algorithms are discussed in Chapter 4 and the data mining algorithms and their functionalities are explained in Chapter 5. The results are provided in Chapter 6 and the summary and conclusions are included in Chapter 7. 


\section{RELATED RESEARCH}

It is convenient to think of data mining systems that were developed during the past decade as comprising three generations: 1) client-server systems; 2) component and agent-based systems; and 3) systems based upon web services. The first generation of data mining system utilized local data, with either client-server or 3-tier architectures. With these systems, a client front end is used to access a server (possibly on the same machine) hosting the data mining application. With a client-server model, the server also manages the data; with a 3-tier model, the data is accessed from another source using ODBC, JDBC, or other related protocol. The next generation of data mining systems was component-based. The components could be local, relying on Microsoft's COM or DCOM platforms, for example, or global, relying on systems such as Suns J2EE platform. Angoss is an example of the former, and Kensington is an example of the latter. More or less at the same time, various experimental agent-based data mining systems were developed. The basic assumption in these systems is that the data is distributed and agents are used to move the data, move the models produced by a local data mining system, or move the results of a local data mining computation. Today, very few agentbased systems are used in practice. This is probably because no agent-based infrastructure, over which an agent-based data mining system must be built, was ever widely adopted. Examples of agent-based distributed data mining systems include JAM [19], Papyrus [7], and BODHI [14]. 
Somewhat later, the next generation of service-based data mining systems began to emerge. These are generally built using W3C's standardization of web services. Examples include DataSpace [9] and data mining systems developed by IBM, Microsoft and SAS that employ the XML for Analysis standard [3]. More general service-based infrastructures, such as grids or data grids [4], are also used for data mining, especially when large computational resources are required. A data grid uses Globus, or an equivalent infrastructure, to provide a security infrastructure and resource management infrastructure so that distributed computing resources can be used. In addition, Globus provides a high performance data transport mechanism called GridFTP. The Grid community has begun an effort called the Open Grid Service Architecture, or OGSA, that provides a web service based access to some grid services [17]. OGSA Database Access and Integration Services (OGSA DAIS) [16] combine grid services with web services for remotely accessing databases.

\subsection{EXISTING SYSTEMS}

There are a few integrated systems developed for distributed data mining. The JAM system developed by Professor Stolfo et al. uses local learning, and outputs from local learning can be combined to build meta-learning. JAM provides a set of learning programs that execute models over data stored locally and also provides a set of agents for combining the results from multiple sites [10]. The Kensington [5] data-mining infrastructure developed by Professor Guo allows access to data from anywhere on the Internet. This remote access to data and mining framework are based on CORBA. The BODHI $[9,10]$ developed by Professor Kargupta is an agent based distributed knowledge 
discovery system. It uses local learning schemes that can be combined at a central location to build meta-knowledge. The Papyrus system [4] developed by Professor Grossman is based on a layered infrastructure for high performance and wide area data mining. The distributed agent based mining environment (DAME) is developed by Krishnaswamy et al. The focus of DAME was to delivery data mining services via the Internet. It can support cluster of workstations connected by high performance network. The client server models for distributed data mining were developed by Chattratichat [2] called DecisionCenter, and the IntelliMiner [13] by Parthasarthy et al. These existing approaches in modeling data mining infrastructure suffer from one or more of the following limitations:

- Selected data mining algorithms are applied independently on different sites and their results are integrated to provide global learning models. Lack of coordination between different sites during the process of local model building affects the quality of results in global learning models [7].

- In all the existing frameworks, a fixed number of distributed data mining algorithms are implemented. This rigid scheme of implementing proprietary data mining algorithms in a framework limits the integration of new improved algorithms developed by third party that are available on the Internet. The data mining framework must be flexible to allow incremental addition to the algorithm knowledge base. 
- The data location and format of data has to be known before hand. In the Internet world data sources are added every day. A data mining framework must have the flexibility to discover new data sources for a particular domain dynamically.

- Most of the existing implementations of data mining infrastructures are tightly coupled and require both ends of the communication system to use the same distributed object model. This may not work across heterogeneous environments and firewall or proxy servers [6].

- Most of the data mining models do not discuss the security/privacy structure for data access and execution of distributed agents. Moreover, there is no discussion of cost / charge for data access and distributed agent execution on the data site.

- Some of the existing frameworks provide an integrated web of data such as DataSpace [4]. These frameworks allow queries to be executed on distributed data sets, but the data types in these frameworks are very limited. The goal of distributed data mining must include web of distributed algorithms and other resources in addition to web of data [5].

In the existing data mining approaches, the success or failure of a distributed datamining project is highly dependent on a particular person and a tool. Successful practice may not necessarily be repeated across the applications, especially when they are becoming more complex and more demanding in a distributed environment [8]. Therefore, data mining needs standard protocols in terms of a methodology and 
technology used. The objective of these standards should be to help user translate an application problem into data mining tasks on a higher abstract level with less efforts [11]. That is especially important for the users in the Internet environment where distributed data mining applications become extremely complex. This complexity increases when requirements for incremental or on-line mining [6] is added to the huge amount of data that is available for mining in distributed heterogeneous systems. Integrating time- and space-sensitive functionality of data in the mining process, and only partial availability of data, confronting privacy and security policies, all that enormously increase the complexity of the data-mining task $[2,8,11]$. This growing complexity of the distributed infrastructure threatens to undermine the very benefits data mining technology aims to provide.

\subsection{Distributed Data Mining (DDM)}

The benefit of understanding large, complex, and information-rich data sets is common to all fields of businesses, science, and engineering. Globally, data mining is defined as a process of discovering various models, summaries, derived nontrivial information, and patterns from a given collection of data $[10,11,13,16]$. The popularity of Internet and Web makes it imperative that data-mining framework is extended to include the distributed and time dependent information and tools. Moreover, the assumptions and the approaches used for static and centralized data mining process are

not valid any more for distributed environment $[14,17]$. Traditional development and deployment of different data mining techniques in a data mining process usually assume that: 
- Data set for analysis is centralized, on a single computer, in a form of centralized database, data warehouse, data mart, or for simple problems as a flat file.

- The amount of available data is enough for successful application of a data mining techniques.

- Software tools supporting different data mining techniques are also centralized; they are located on the same computers where the data are stored.

For many classical data mining applications these assumptions are good enough $[10,11,12]$ but in an Internet-based distributed information environment these assumptions about data, algorithms, and data mining process are not true and may represent constraints on the quality of data mining results. Standardization on one side, and use of new technologies for an infrastructure improvement on the other side, will make large, complex data mining projects less costly, more reliable, more repeatable, more manageable, more efficient, and very important, with new higher quality in data mining results. Our approach in standardization of distributed data mining methodology is based on a model of data mining process having four levels of abstraction [15]. They are organized in a hierarchical structure: a) phases of data mining, b) generic tasks, c) specialized tasks, and d) process instances. The hierarchy of the model is graphically presented in Figure 2.1.

The structure will be illustrated with examples of task hierarchies shown in Figure 2.2. For example, in the exploration phase one of generic tasks is "build the model", while its specialized task is "build classification model", and corresponding process instances are: "neural network", "decision rules", or "logistic regression". The 
other example of task hierarchy is data preparation phase, where "data cleaning" is a generic task, possible specialized task is "elimination of missing values", and corresponding process instances are: "mean value algorithm" or "clustering".

While higher level of abstract concepts in the hierarchy explain what to do, lower level components go in details of data processing and answer the question how to do. We have used the bottom-up approach for building service-oriented infrastructure for data mining. The implementation starts with process instances as components of Web services, and then integrate them into more abstract, and at the same time more complex and intelligent data mining specialization tasks.

\section{Data Mining Process}

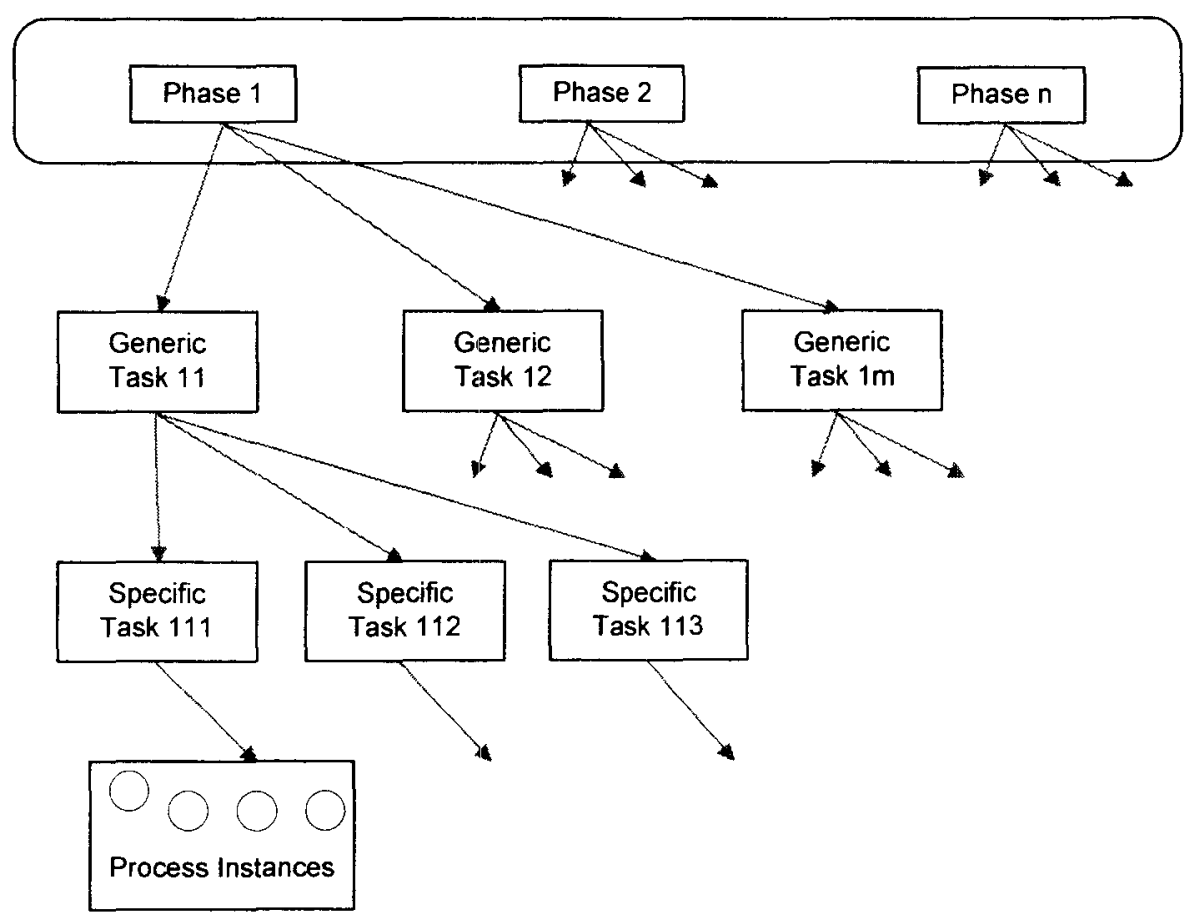

Figure 2.1: Hierarchical structure of a (Distributed) Data Mining Model 

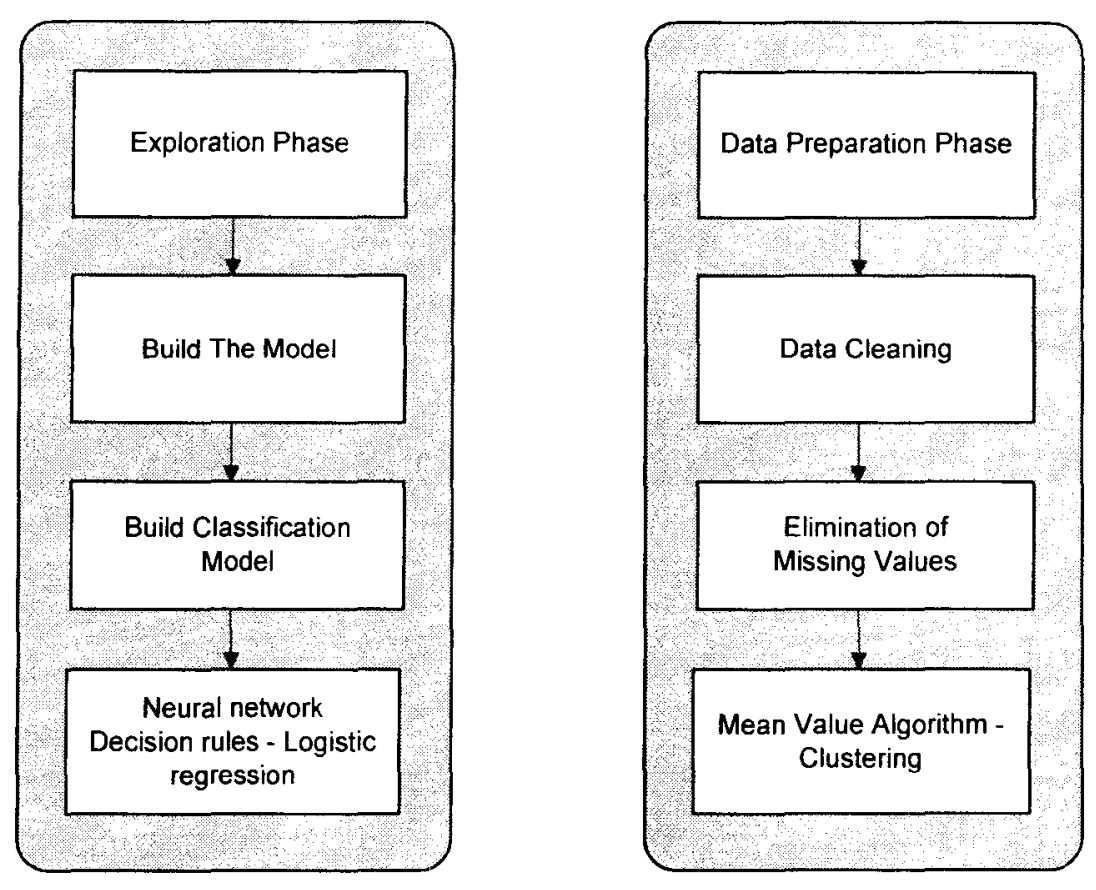

Figure 2.2: Examples of paths in hierarchical structure of a data mining process

\subsection{Web SERVICES}

A term that refers to distributed or virtual applications or processes that use the Internet to link activities or software components. Web services use the following standards for communication and data processing:

- XML (eXtensible Markup Language) - An open standard for describing data.

- UDDI (Universal Description, Discovery, Integration specification) - Descriptive standard for documentation and how/where to publish it in an automated fashion.

- SOAP (Simple Object Access Protocol) - A technique to allow communications between applications over the web.

- WSDL (Web Services Description Language) - An XML specification for describing web services, what they do, and how to access them. 
A web service makes itself available by describing itself in a Web Services Description Language (WSDL) document. WSDL document is a XML document that has all the information about web service, including its name, the operations it supports, parameters for those operations and the location where it is running. For service consumers or clients to locate the web service, web service provider has to publish the web service at registry server. Universal Description, Discovery, and Integration (UDDI) are a standard protocol to publish or to find web services. The description part in UDDI is for service provider to publish details about their organization and web services they provide. The discovery part in UDDI is for service consumers or clients to find these services. Service consumer or client invokes web service using Simple Object Access Protocol (SOAP). SOAP is a lightweight XML protocol used for information exchange between heterogeneous systems.

- Service provider registers the service in UDDI Registry.

- Client finds the service, gets the WSDL for the service.

- Using WSDL, client constructs a web service call and invokes the service.

\section{Advantages of Web Services:}

1. Interoperability: Client written in Java can invoke a service written in .NET or vice versa.

2. Ubiquity: Once the web service is registered in a registry server, any organization can use it.

3. Loosely coupled: Web service does not depend on the underlying system architecture. 
Creating a Distributed Data Mining architecture using Web services involves a lot of new technologies. Java Web Services Developer Pack (JWSDP) from Sun Microsystems solves many of the issues and was suitable for this framework and hence chosen as the toolkit for its implementation.

\subsection{SOAP BASED WEB SERVICES}

A web service may be implemented as a standalone TCP server, or it may be accessed via a URL through a web server. When running under a web server, the SOAP service can take advantage of firewall tunneling, although performance will be reduced. The SOAP service accepts XML that describes an action for the server to perform, and returns XML to the client describing the result of the operation. It is possible to maintain state between operations, and operations are essentially non-streaming, due to the marshaling rules of XML. There are two fundamental problems when using web services for data mining of moderate to large size remote and distributed data sets. First, due to the overhead of XML encoding and parsing, there is a limit to the speed of the data transmission and the total size of the return set. This is caused by the need to retain the entire dataset in local storage due to XML encoding and decoding rules. The specific issue is that redundant parts of XML documents can and must refer to the other similar parts of documents. This requires that the entire document be maintained for lookup purposes. Therefore, all data packaging mechanisms that are truly XML compliant are in essence non-streaming. While a server could, in theory, safely ignore this encoding rule when there are no circular data structures, a compliant client cannot safely do so. 


\section{Web BaSed Distributed Data Mining Framework}

Distributed environment used to be viewed as a solution for intensive computations with large datasets. However, operating with real world applications in distributed environment motivated new reasons to appear different than the traditional one. One application is in distributed data mining. Data mining algorithms are known with their combinatorial results which make distributed environment a good candidate for them. But sometimes, distribution of data is intended because the distribution itself has a meaning. A very popular example is in banking where banks like to exchange their models for fraud detection or loans approval. This is infeasible because of privacy of customers. Even if exchanging models were feasible it will lead to unexpressive results in case of loans approval because banks apply different policies for loans. In these cases, the following approach for mining distributed data is suggested:

- Build local models for each site

- Discuss relations between local models of sites i.e., whish sites are similar and which are different, to what extent they differ and the implication and significance of their differences.

- For similar sites build global model(s) and exclude those very different 


\subsection{SERVICE ORIENTED APPROACH}

Service oriented computing has become ever more popular due to the proliferation of web based computing [6]. In this paradigm, core Web Services [11] (as Web Components) form the fundamental elements for developing large and complex data mining environment. The core services, their description, operations including publication, discovery, selection and binding constitute the foundation of service oriented computing. Using these core services higher composition layers encompassing functionality of multiple layers at lower level can be built. The aggregate service components can then become part of the core services and can be used in building higherlevel components. These aggregate components can be published and discovered by applications for providing effective solutions to users.

In general, service oriented Web Components are self-describing, based on open standards, allow rapid composition of distributed applications. Service or component providers develop these service-based components; they supply the detailed description of the functionality through the Web Service Definition Language (WSDL). The data or algorithm providers register the services with descriptions WSDL in the registries and the registries are searched by the clients for the required Web Services components $[11,15]$.

These components provide distributed computing framework across different platforms and languages. The design of core Web Services and aggregate services is a distributed programming task where service providers want to reuse existing services by extending or restricting their functionality without building from scratch. Currently, there 
is no data mining framework that allows the definition and implementation of data mining Web Services compositions. In order to alleviate this limitation, a framework for developing aggregate services for distributed data mining is proposed in this project.

\subsection{EXECUTION FRAMEWORK}

The design of core Web services and aggregate services is a distributed programming task where service providers want to reuse existing services by extending or restricting their functionality without building from scratch. Currently, there is no data mining framework that allows the definition and implementation of data mining Web service compositions. In order to alleviate this limitation, a framework for developing aggregate services for Distributed Data Mining is proposed in this paper.

In order to build service oriented framework for distributed data mining, we propose the architecture shown in Figure 3.1. In subsequent discussion, first the major components and their interaction are discussed followed by the details of major modules required for this architecture. In this framework data and algorithm providers will register their resources as follows:

Algorithm and data providers will describe their services using Web Services Description Language (WSDL) and interact with the registration service. Registration service will use WSDL description for entry in the registry. 
Clients will interact with the Web interfaces and provide their data mining requirements in terms of data to be analyzed and types of mining activities to be performed. Detailed structure of data and the structure of algorithm are obtained from the registry by retrieving the WSDL for the data set and algorithm. Based on the client selection of data and algorithm, the execution framework will generate the execution sequence and will interact with the data and algorithm provider directly based on the information in WSDL.

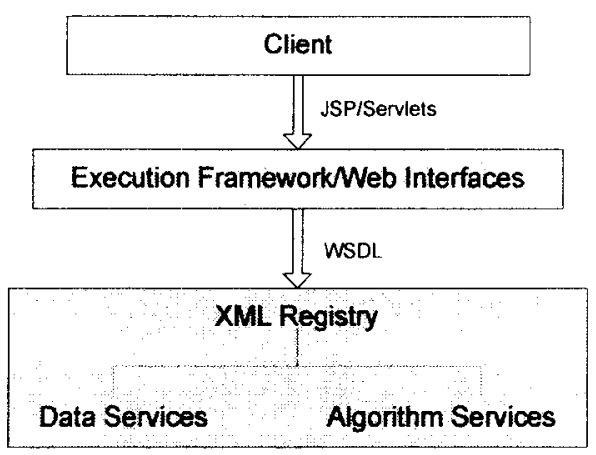

Figure 3.1: Execution framework

\subsection{DETAILED ARCHITECTURE}

In order to build service oriented framework for distributed data mining, we propose the architecture shown in Figure 3.2. In subsequent discussion, the major components and their interaction are discussed followed by the details of major modules required for this architecture. The core components of the proposed framework are described in Table 3.1. 


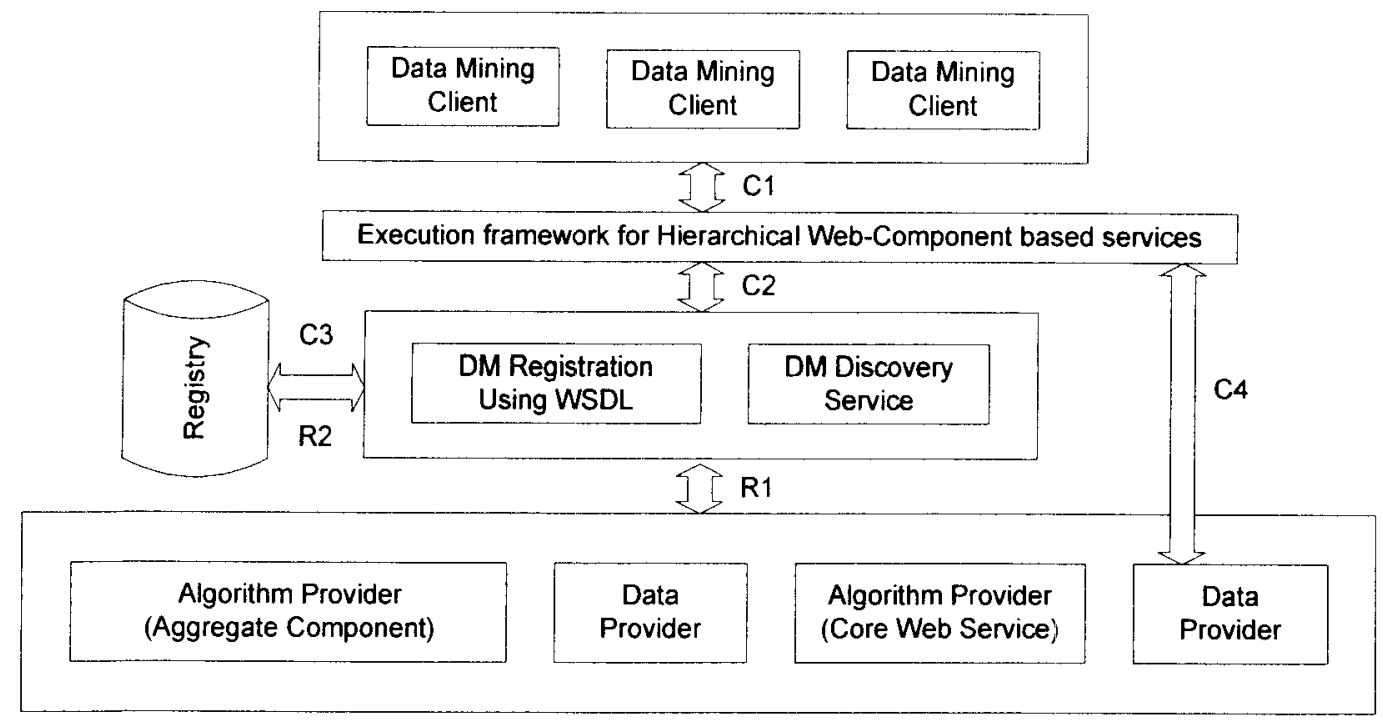

Figure 3.2: Architecture for hierarchical Web Component service execution

\begin{tabular}{|c|c|}
\hline Component & Functionality \\
\hline Clients & The user of the distributed data-mining framework. \\
\hline Registry & $\begin{array}{l}\text { This location has database for all the algorithm service } \\
\text { providers and the data provides a to keeps their details in } \\
\text { the form of WSDL This registry could be a local registry } \\
\text { in an organization, or global UDDI registries [8]. }\end{array}$ \\
\hline $\begin{array}{l}\text { Data Mining } \\
\text { Discovery } \\
\text { Service }\end{array}$ & $\begin{array}{l}\text { Allows clients to discover and select distributed data sets } \\
\text { and algorithms based on data mining problem } \\
\text { requirement and returns WSDL description to Data } \\
\text { Mining Web component execution framework. }\end{array}$ \\
\hline $\begin{array}{l}\text { Algorithm } \\
\text { Provider }\end{array}$ & $\begin{array}{l}\text { Provides the data mining algorithms. These algorithms } \\
\text { could be core Web Services or aggregate components. It } \\
\text { is the responsibility of the provider to register the WSDL } \\
\text { of the dataset in registry using registration service. }\end{array}$ \\
\hline Data Provider & $\begin{array}{l}\text { Provides the datasels for analyses. It is the responsibility } \\
\text { of the provider to register the WSDL of the dataset in } \\
\text { registry using registration service. }\end{array}$ \\
\hline $\begin{array}{l}\text { Execution } \\
\text { Framework }\end{array}$ & $\begin{array}{l}\text { Provides support for invoking data and algorithm } \\
\text { discovery service and the proper execution of selected } \\
\text { algorithms. }\end{array}$ \\
\hline
\end{tabular}

Table 3.1: Functionality of the core components in the proposed framework given in Figure 3.2 
In this framework data and algorithm providers will register their resources as follows:

R1: Algorithm and data providers will describe their services using Web Services Description Language (WSDL) and interact with Registration service.

R2: Registration service will use WSDL description for entry in the registry.

Clients will interact with data mining execution framework as follows:

C1: Clients will interact with Web Component execution framework and provide their data mining requirements in terms of data to be analyzed and types of mining activities to be performed.

C2: This framework will interact with data mining discovery service application program interface (API) by specifying data and algorithm requirements.

C3: The discovery service will provide a set of data and algorithms matching the client's requirement. Client will select the resources for data mining. Detailed structure of data and the structure of algorithm are obtained from registry by retrieving the WSDL for the data set and algorithm.

C4: Based on the client selection of data and algorithm, the Web component execution framework will generate the execution sequence and will interact with the data and algorithm provider directly based on the information in WSDL. 


\section{IMPLEMENTATION FRAMEWORK}

\subsection{DetaILED SySTEM ARCHITECTURE}

Registration is the fist step toward system initiation, during which services are registered with the registry. The services can be either data services or algorithm services. The registration process requires the following details from the service provider.

- Organization: Name and Description

- Contact: Name, Phone, and Email

- Service: Name and Description

- Service Binding: URI and Description

An organization can have one service with several bindings or multiple services. Figure 4.1 shows the registration process.

This data registration process requires the data providers to specify the fields that are available for performing mining tasks, the data repository location, and how to get access the data or execute an algorithm on the data site. In the algorithm registration process, the provider gives the detailed information about the parameters associated with the service and other configuration details. 


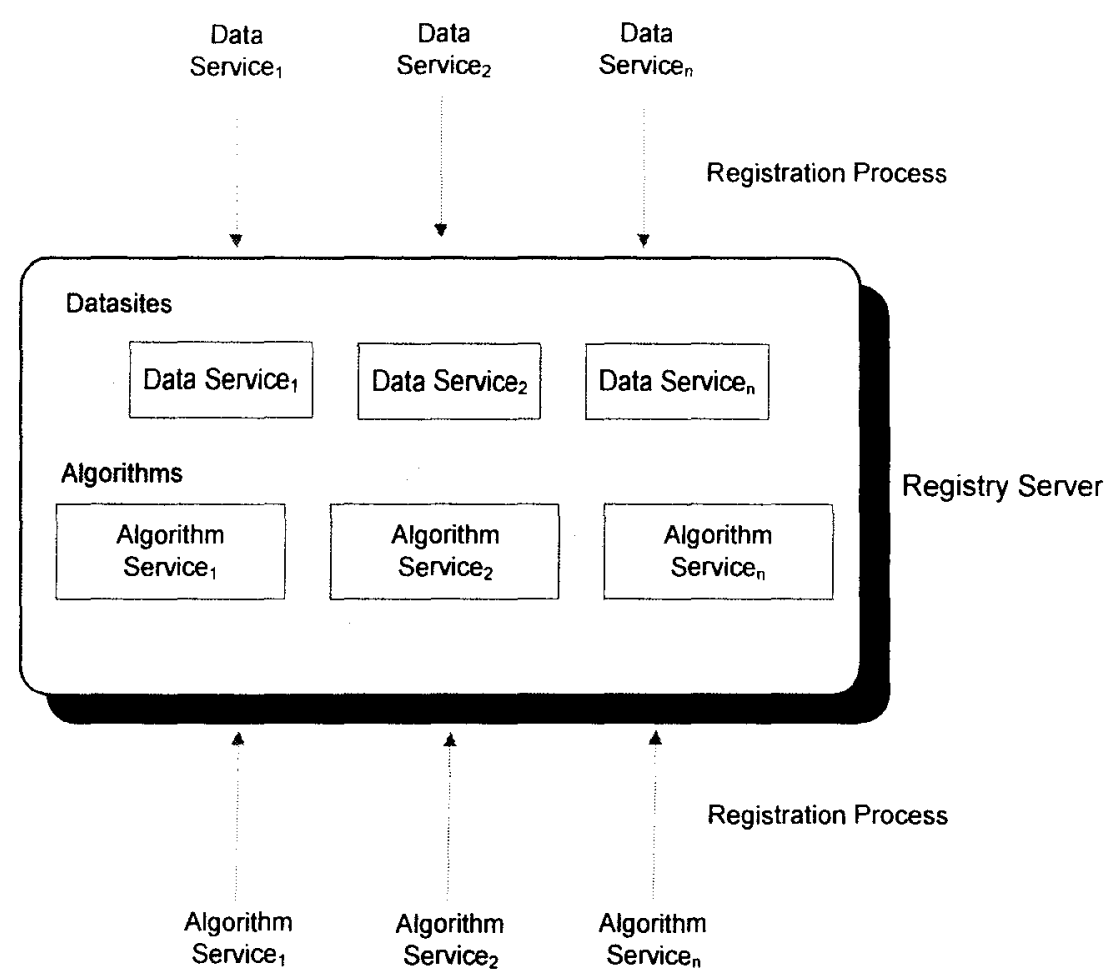

Figure 4.1: The registration process where the service providers register their services.

Once the registration process is complete, the services are ready to be used by the system for applying the various algorithms on the registered data sites. The first step of processing a client request is to select the dataset the client is interested in. In the proposed framework, a client is allowed to select a dataset from a list of available registered data services of their interest. Once the dataset is chosen the client has a choice of algorithms that can be applied to the selected dataset. All the dataset and algorithm information is retrieved from the registry.

In data registration, the data providers specify the data fields that are available for mining, the location of the data, and how to get access the data or execute an algorithm on the data site. In algorithm registration, the provider gives the detailed information 
about the service execution. For example information included for Fractal Dimension service is shown in Table 4.1.

\begin{tabular}{|ll|}
\hline Attribute & Value \\
\hline Organization Name & UofL ASP \\
Organization Description & University of Louisville - Algorithm Service Providers \\
Service Name & Fractal Dimension \\
Service Description & Algorithm Service to calculate Fractal Dimension, number and types of \\
& inputs needed, outputs generated \\
Service Binding URI & http://136.165.147.86:8080/fdalgo-jaxrpc/fd \\
Service Binding Description & Service Binding for Fractal Dimension Algorithm \\
\end{tabular}

Table 4.1: Registration information for a service

Once the registration process is complete, the services are ready to be used by the system for applying the various algorithms on the registered data sites. The first step of processing a client request is to select the dataset the client is interested in. In the proposed framework a client is allowed to choose a dataset from a list of available registered data set services of their interest. Once the dataset is selected the client has a choice of algorithms that can be applied to the selected dataset. All the dataset and algorithm information is retrieved from the registry.

Figure 4.2 describes the general structure of most distributed data mining algorithms. In this figure distributed data mining algorithms are broken in three different parts that could be executed iteratively. The first part of the logic is performed on all the data sets involved for computing local models. These intermediate results are then 
integrated in part 2 of the processing model. Then, the integrated results are sent to part 3 of the computation that will distribute results for generating global models of data mining algorithm. One of the main goals in building distributed data mining algorithms is to reduce the amount of data exchanged between computing sites.

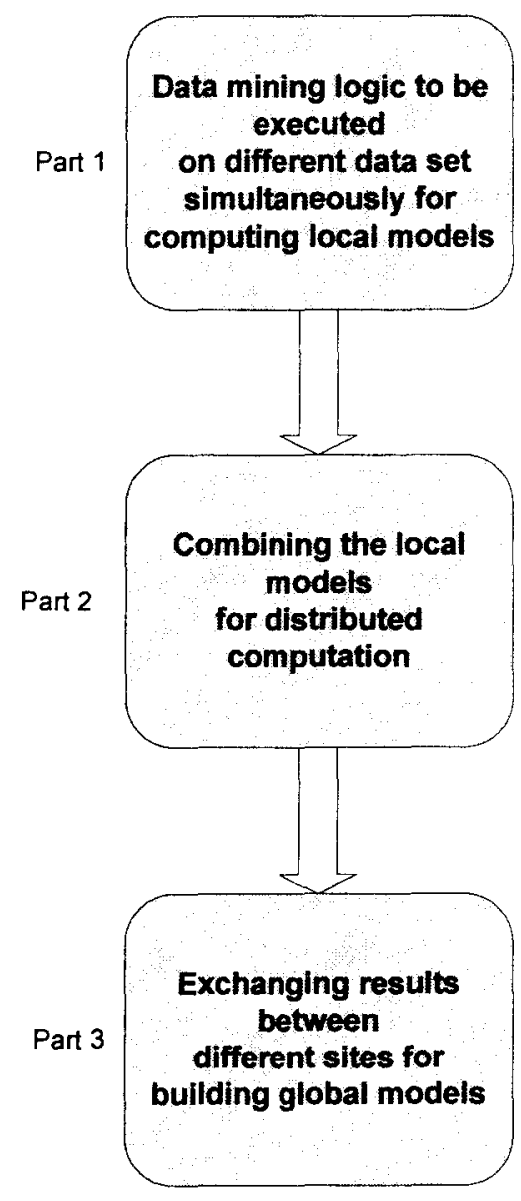

Figure 4.2: Structure of distributed data mining approach for distributed data

In our implementation framework parts 2 and 3 of the DDM service are generic, and will be offered as a core sub-service. This sub-service can perform different types of activities on various results obtained at different sites. 
The implementation is broken in two parts:

i) the logic to be executed independently on various data sets.

ii) the logic that needs to be executed with the combined results.

We have chosen two approaches to implement this framework:

\section{a) Centralized approach}

In the centralized approach, the datasets residing on remote sites are collected and combined to form one data resource bundle on which the algorithm is executed as shown in Figure 4.3.

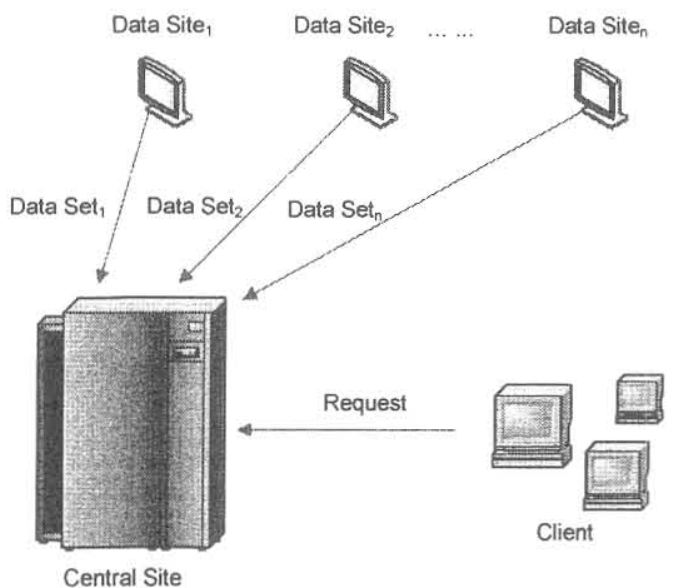

Figure 4.3: The datasets being collected into the central site and the results being sent to the client after processing the global model

\section{b) Distributed approach}

In the distributed approach, the datasets are not moved and they always reside on the remote sites. The algorithm is propagated to the remote data sites where it processes the individual dataset and returns local models to a coordination site. The results are then processed to generate the global model and thereafter sent to the client. The number of 
intermediate results directly depends on the data mining algorithm itself. The distributed approach may require multiple transfers of data between the co-ordination site and the data sites but it eventually proves to be the better of the two choices, especially for large real world data sets since the amount of data transferred is considerably lesser compared to the fetching of the actual datasets. The distributed approach is shown in Figure 4.4.

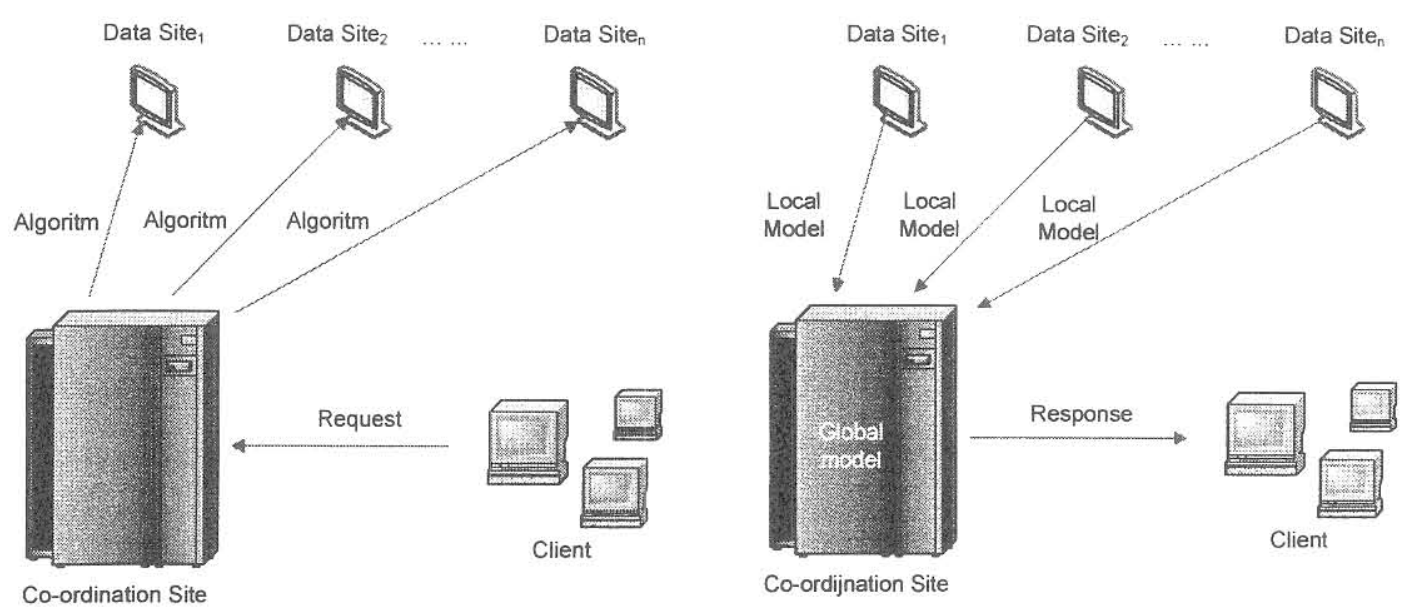

Figure 4.4: The algorithm being sent to the remote site and local models being collected to generate the global model and results being sent to the client after processing the global model

\subsection{IMPLEMENTATION OF THE SERVICE FRAMEWORK}

Registering Data and Algorithm Services require the following common parameters:
a. Service Name
b. Service Description
c. Service URI
d. Classification

The classification is an optional attribute but it plays a major role in reducing drastic amounts of search time. 


\subsubsection{Data Services}

The data service registration is a two step process. The first step requests the user to provide the service information and the number of data fields that are available for processing. Figure 4.5a shows the interface the client uses as the first step towards registering the data service.

\section{Data Service Registration (Step 1)}

Service Hame
Service Description
Serrice URI
Classitication

Please specify the classification if it is not listed.

Number of fields

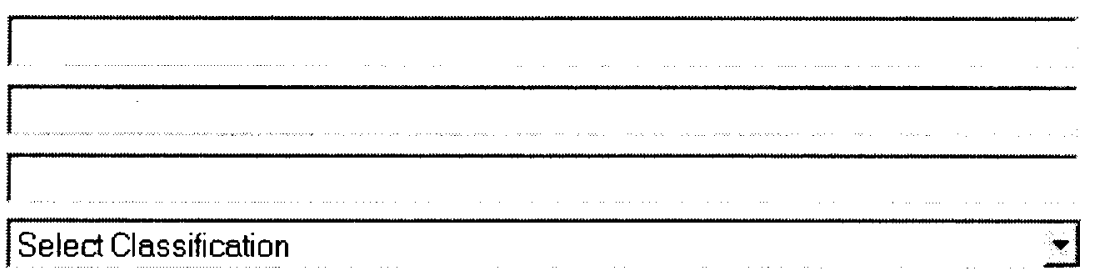

Next $>$

Figure 4.5a: Step 1 of the Data Service registration process

For each attribute of the data service, the following parameters need to be specified.
a) Attribute Name
b) Attribute Type
c) Attribute Cost
d) Attribute Exposure

The Attribute Type can be any of the data types supported by Web Services. The Attribute Cost specifies the amount to use the attribute. The Attribute Exposure specifies if the user can view this attribute. A value of Yes allows the user to view/copy the attribute. A value of No means that the attribute can be used to test algorithms on but the 
actual values cannot be viewed or copied. Figure $4.5 \mathrm{~b}$ shows the interface the client uses as the second step to provide this information about the data service. Suppose the Number of fields was set to 5 in Step 1.

\section{Data Service Registration (Step 2)}

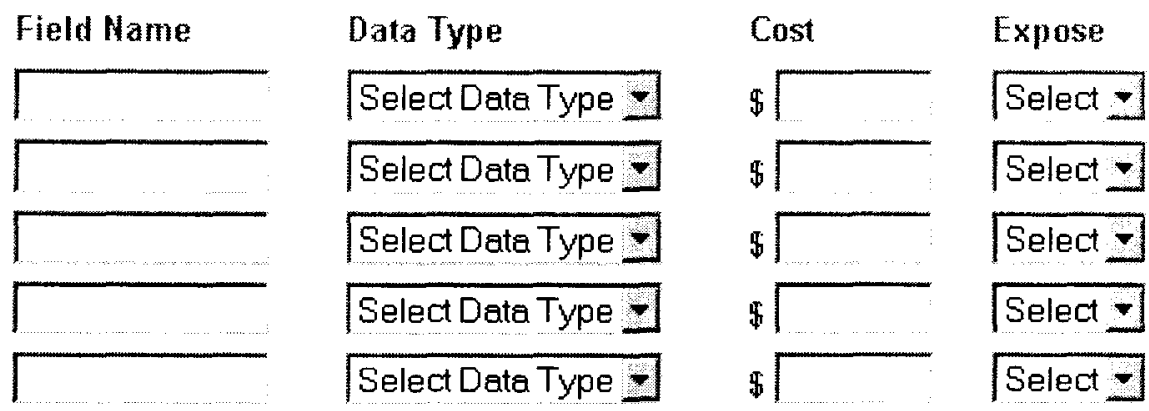

\section{Next $>>$}

Figure 4.5b: Step 2 of the Data Service registration process

The information provided is used to register the Service with the local registry and additional information is stored in a database. Figure 4.5c shows the registry response received once the data service registration is complete.

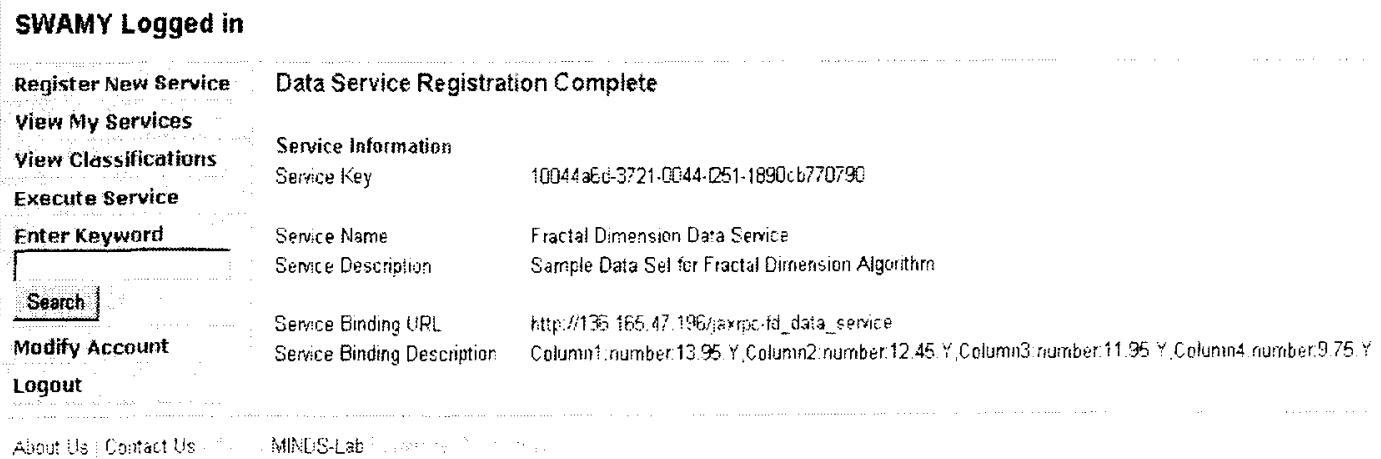

Figure 4.5c: Registry response for the Data Service registration process 


\subsubsection{ALgORITHM SERVICES}

The algorithm service registration is a two step process. The first step requests the user to provide the service information and the cost of using the algorithm and the number of input parameters accepted. Figure 4.6a shows the interface the client uses as the first step towards registering the algorithm service.

\section{Algorithm Service Registration (Step 1)}

Semice Name
Service Description
Service URI
Classification

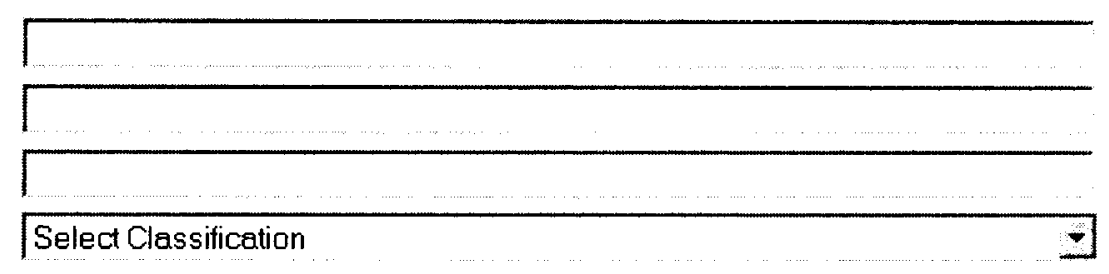

Please specify the classification if it is nol listed.

Algorithm Cost

Number of input parameters

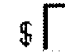

Next $>$

Figure 4.6a: Step 1 of the Algorithm Service registration process

The second step of the algorithm service registration process requires the following additional fields:
a) Number of input parameters
b) Output parameter type
c) Algorithm Cost

Based on the parameter Number of input parameters, Step 2 will dynamically allocate the required number of parameters to accept the data type. Figure 4.6b shows the interface the client uses as the second step to provide this information. Suppose the Number of input parameters was set to 5 in Step 1. 


\section{Algorithm Service Registration (Step 2)}

$\begin{array}{ll}\text { Parameter } & \text { Data Type } \\ \text { Input-1 } & \text { Select Data Type } \\ \text { Input-2 } & \text { Select Data Type } \\ \text { Input-3 } & \text { Select Data Type } \\ \text { Input-4 } & \text { Select Data Type } \\ \text { Input-5 } & \text { Select Data Type }\end{array}$

Output Parameter

Select Data Type $\mathbf{x}$

Next $>>$

Figure 4.6b: Step 2 of the Algorithm Service registration process

The information provided is used to register the Service with the local registry and additional information is stored in a database. Figure 4.6c shows the registry response received once the algorithm service registration is complete.

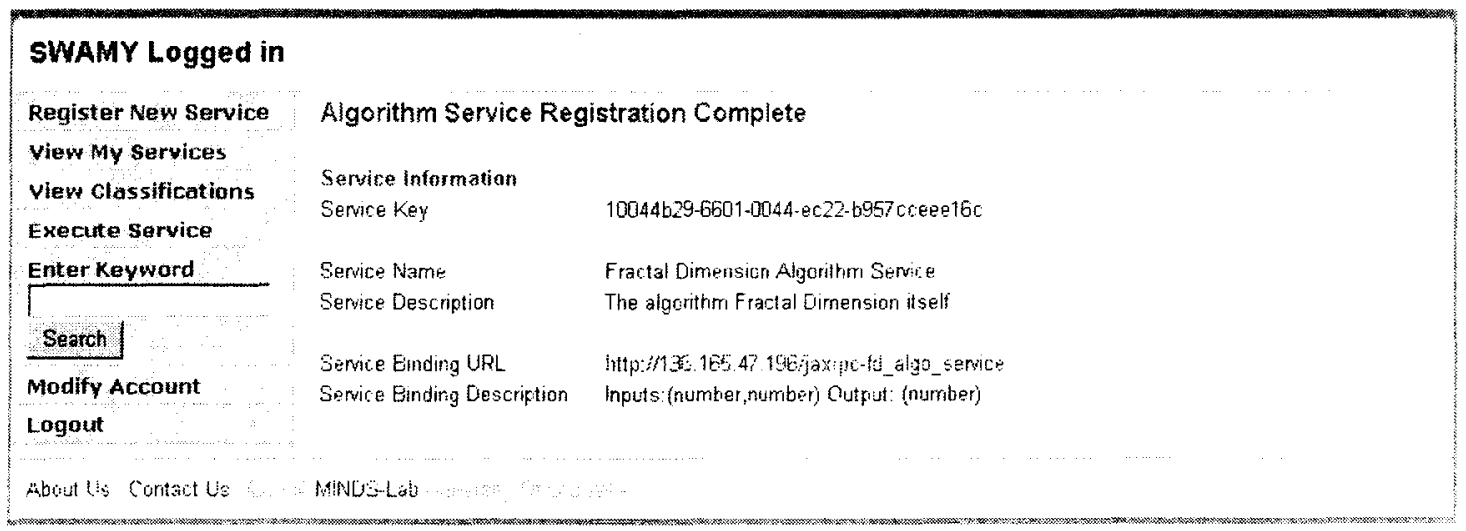

Figure 4.6c: Registry response for the Algorithm Service registration process 


\subsubsection{Centralized Services}

In the centralized approach, a collection of remote datasets is performed concurrently to form a single data resource bundle on which the data mining algorithm is executed. The algorithm also needs to be fetched from its remote location to the coordination site where the actual execution takes place. The following web services are used to achieve the execution of the centralized framework.

- CopyRemoteData (in remote_location, in data_handler, in local_repository): reads the data from the "remote location" based on the specifications of the data handler" and stores the data in the "local_repository"

- CombineData (in local_repository, out combined_data): combines the data read from the "local_repository" to create one large data source, "combined_data".

- FetchAlgorithm (in algorithm_location): fetches the data mining algorithm from the "algorithm location" and stores it locally for processing the data in the local repository

- ExecuteAlgorithm (in local_repository, out results): processes the data mining algorithm on the "local repository" with the logic specified in the algorithm. Returns the intermediate "results" for performing other tasks or the final "results". 


\subsubsection{DiSTRIBUTED SERVICES}

In the distributed approach, the datasets always reside on the remote sites and are never moved. The algorithm is propagated to the remote data sites where it processes the individual dataset and returns local models to a coordination site. The results are then processed to generate the global model and thereafter sent to the client. The number of intermediate results directly depends on the data mining algorithm itself.

- SendAlgorithm (in algorithm_location, in remote_locations): fetches the data mining algorithm from the "algorithm_location" and sends it to the "remotelocations" for processing the data.

- ExecuteAlgorithm (in remote_locations, out results): executes the algorithms concurrently on the "remote_locations" and stores the "results" on the remote location as the data model.

- CopyRemoteModel (in remote_models): reads the "remote_models" from the remote locations and copies it to the local coordination site for processing to get the final results.

The distributed approach may require multiple transfers of data between the coordination site and the data sites but it eventually proves to be the better of the two choices, especially for large real world data sets since the amount of data transferred is considerably less compared to the fetching of the actual datasets. The performance improvement can be measured in terms of execution time as well as the quality of service. 


\subsection{Selecting Dataset ANd Algorithm Service Information}

The first step in the exceution phase is to choose the desired datasets and the algorithm that can be applied on them. Figure 4.8 shows the steps involved in the selection of dataset and algorithm information from the registry.

The client uses the web interface to query the registry for information about available datasets based on classifications or keyword search. The registry returns the appropriate information on all the datasets that match the search criteria. Once the client selects the datasets for processing, a search for algorithms that match the selected datasets is performed and the algorithm service information that can be applied on them is displayed. This step of the implementation is common for the centralized and distributed approach.

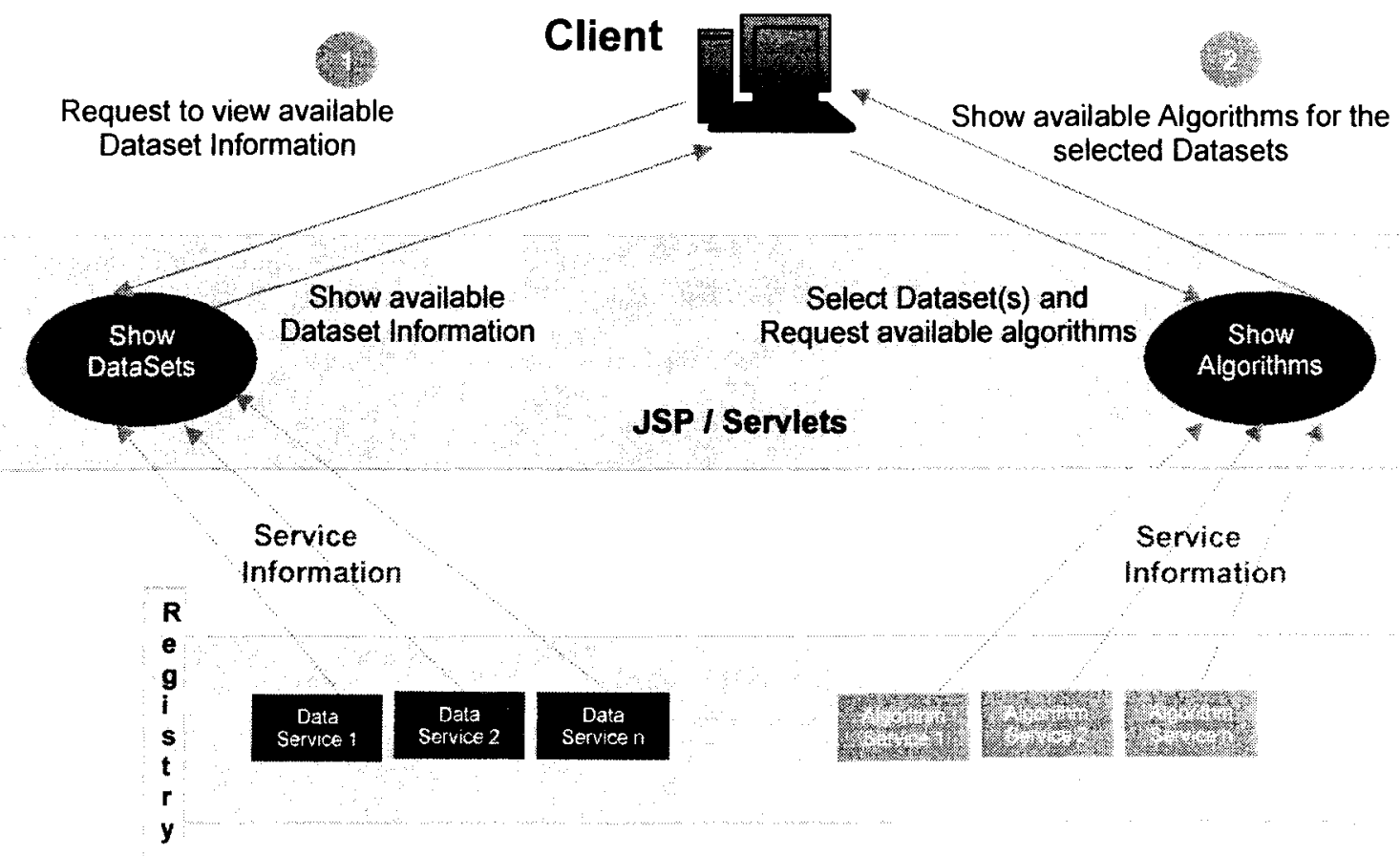

Figure 4.8: The client requests to view service information from the registry 


\subsubsection{EXeCUtion - Centralized APProaCh}

Step 1: The client invokes the data mining services from a URL. The client selects the required datasets located on remote sites.

Step 2: Servlet calls the service on the selected data sites to collect the data. The collected data is stored in a centralized location for processing as shown in Figure 4.9.

Step 3: The Apriori Service downloads the algorithm to the central site and is applied to build the global model for the datasets at the central site.

The datasets are now available as a single resource in the central site. The algorithm is applied on this combined data set and the results are returned to the client.

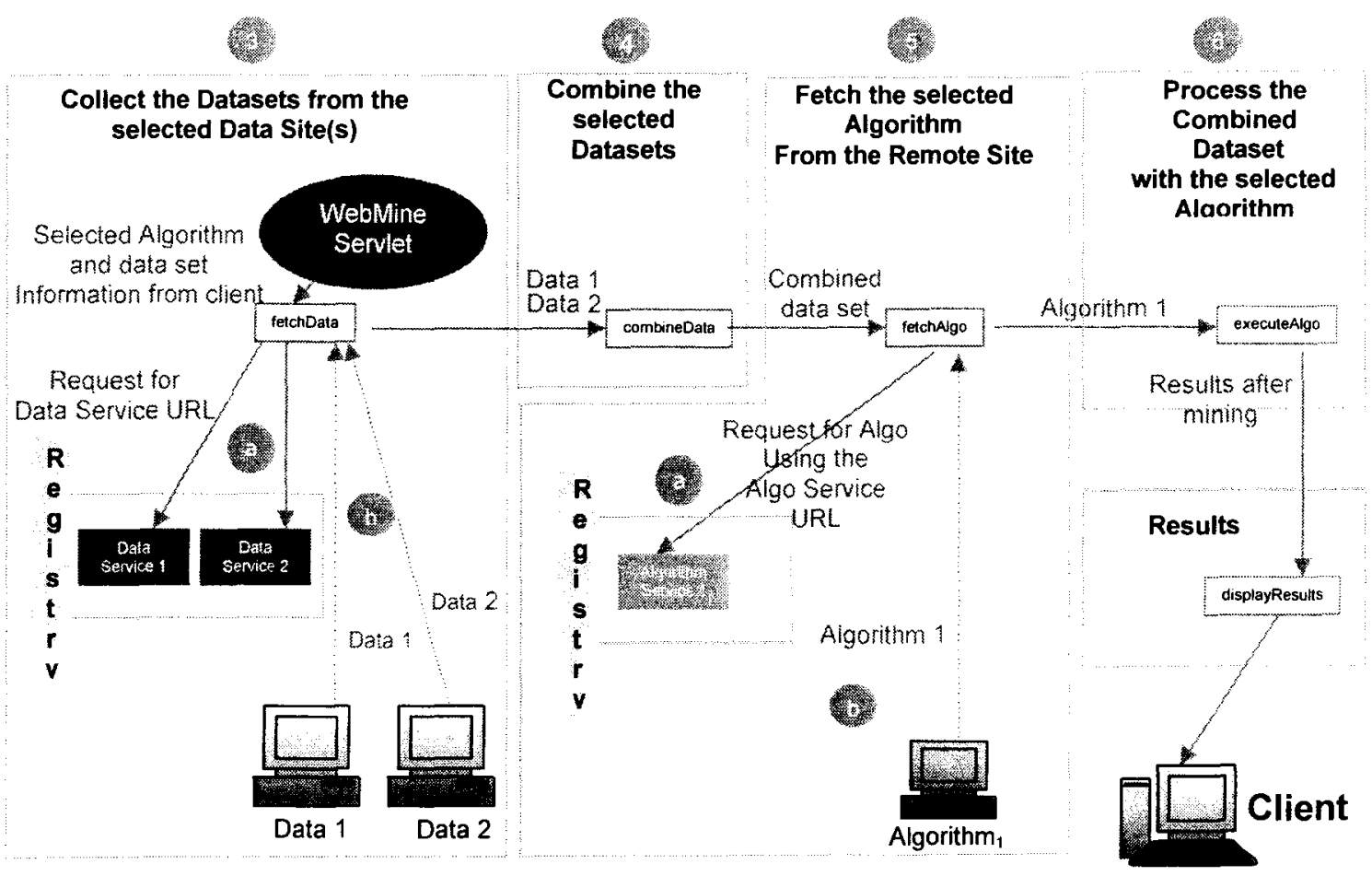

Figure 4.9: Client interaction in Centralized Approach 


\subsubsection{Data Services}

The data service registration is a two step process. The first step requests the user to provide the service information and the number of data fields that are available for processing. Figure 4.5a shows the interface the client uses as the first step towards registering the data service.

\section{Data Service Registration (Step 1)}

Service Hame
Service Description
Serrice URI
Classitication

Please specify the classification if it is not listed.

Number of fields

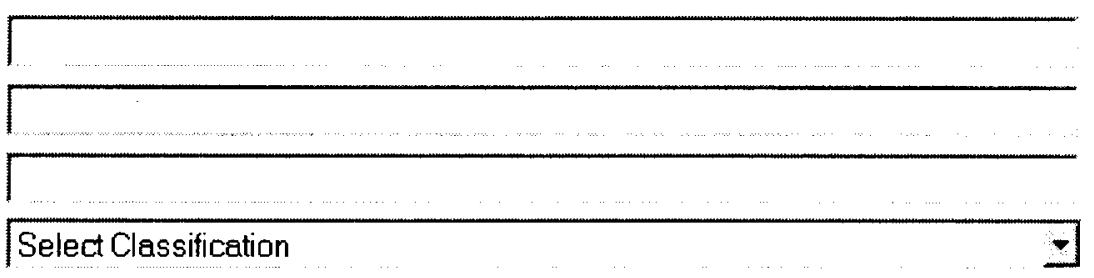

Next $>$

Figure 4.5a: Step 1 of the Data Service registration process

For each attribute of the data service, the following parameters need to be specified.
a) Attribute Name
b) Attribute Type
c) Attribute Cost
d) Attribute Exposure

The Attribute Type can be any of the data types supported by Web Services. The Attribute Cost specifies the amount to use the attribute. The Attribute Exposure specifies if the user can view this attribute. A value of Yes allows the user to view/copy the attribute. A value of No means that the attribute can be used to test algorithms on but the 
actual values cannot be viewed or copied. Figure $4.5 \mathrm{~b}$ shows the interface the client uses as the second step to provide this information about the data service. Suppose the Number of fields was set to 5 in Step 1.

\section{Data Service Registration (Step 2)}

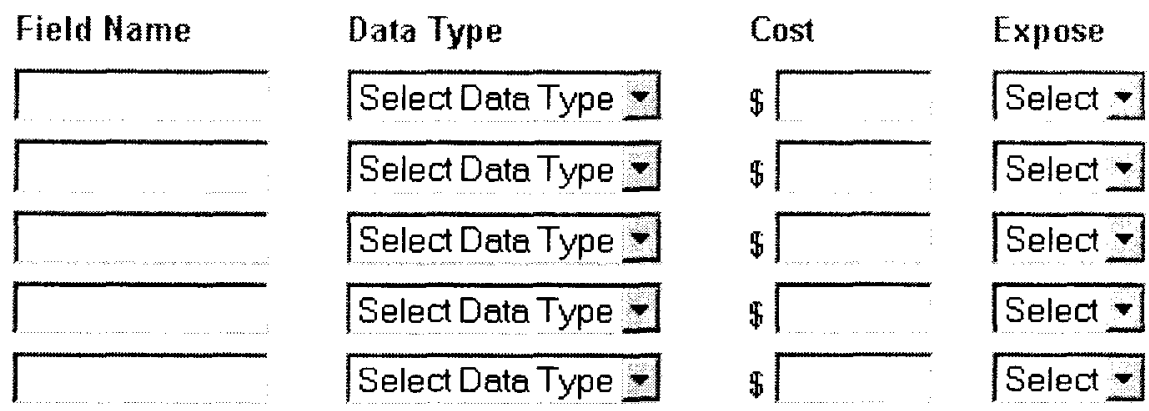

\section{Next $>>$}

Figure 4.5b: Step 2 of the Data Service registration process

The information provided is used to register the Service with the local registry and additional information is stored in a database. Figure 4.5c shows the registry response received once the data service registration is complete.

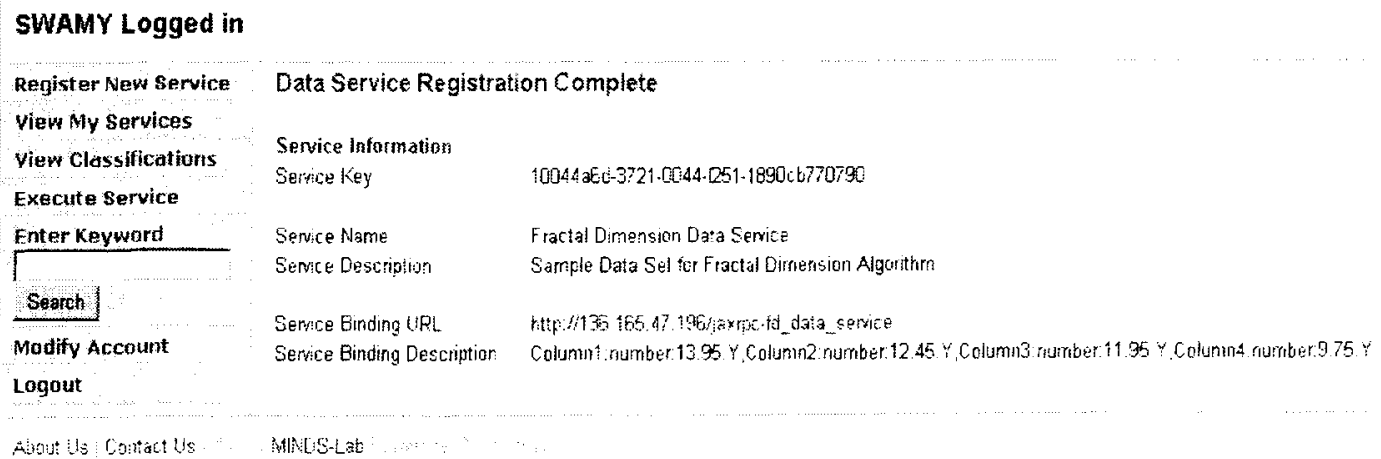

Figure 4.5c: Registry response for the Data Service registration process 


\subsubsection{ALgORITHM SERVICES}

The algorithm service registration is a two step process. The first step requests the user to provide the service information and the cost of using the algorithm and the number of input parameters accepted. Figure 4.6a shows the interface the client uses as the first step towards registering the algorithm service.

\section{Algorithm Service Registration (Step 1)}

Semice Name
Service Description
Service URI
Classification

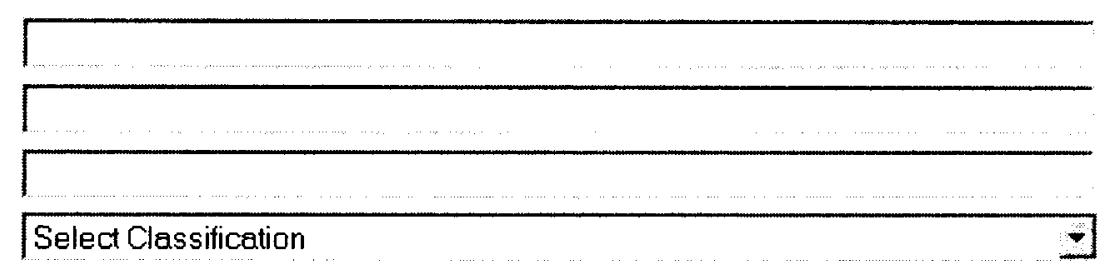

Please specify the classification if it is nol listed.

Algorithm Cost

Number of input parameters

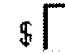

Next $>$

Figure 4.6a: Step 1 of the Algorithm Service registration process

The second step of the algorithm service registration process requires the following additional fields:
a) Number of input parameters
b) Output parameter type
c) Algorithm Cost

Based on the parameter Number of input parameters, Step 2 will dynamically allocate the required number of parameters to accept the data type. Figure 4.6b shows the interface the client uses as the second step to provide this information. Suppose the Number of input parameters was set to 5 in Step 1. 


\section{Algorithm Service Registration (Step 2)}

$\begin{array}{ll}\text { Parameter } & \text { Data Type } \\ \text { Input-1 } & \text { Select Data Type } \\ \text { Input-2 } & \text { Select Data Type } \\ \text { Input-3 } & \text { Select Data Type } \\ \text { Input-4 } & \text { Select Data Type } \\ \text { Input-5 } & \text { Select Data Type }\end{array}$

Output Parameter

Select Data Type $\mathbf{x}$

Next $>>$

Figure 4.6b: Step 2 of the Algorithm Service registration process

The information provided is used to register the Service with the local registry and additional information is stored in a database. Figure 4.6c shows the registry response received once the algorithm service registration is complete.

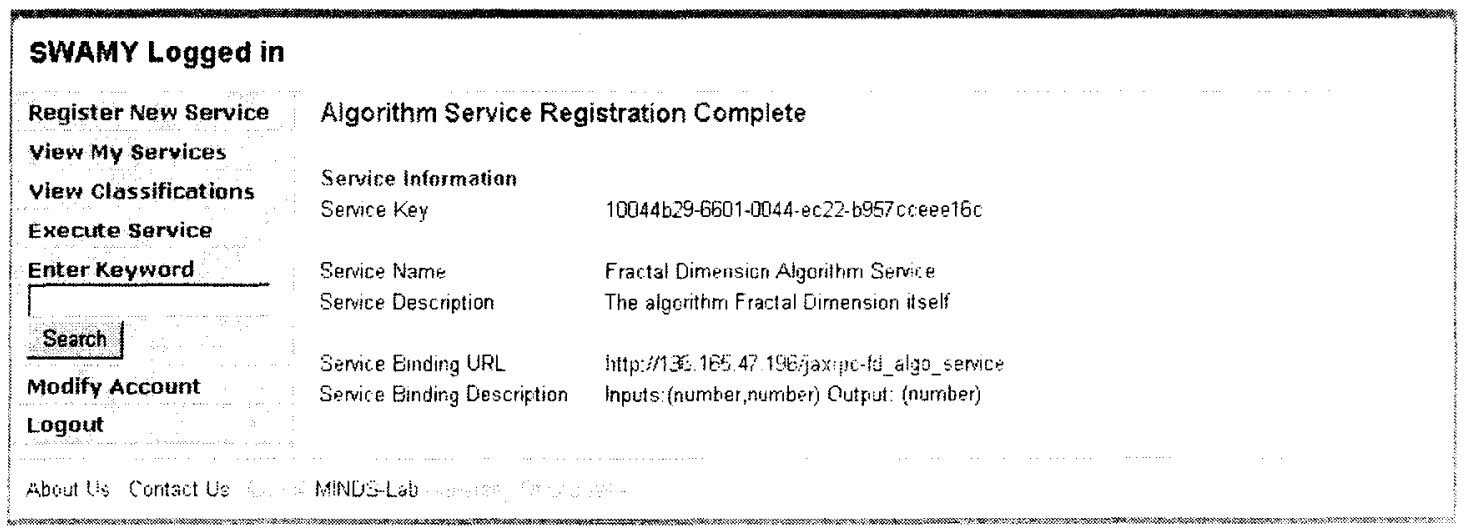

Figure 4.6c: Registry response for the Algorithm Service registration process 


\subsubsection{Centralized Services}

In the centralized approach, a collection of remote datasets is performed concurrently to form a single data resource bundle on which the data mining algorithm is executed. The algorithm also needs to be fetched from its remote location to the coordination site where the actual execution takes place. The following web services are used to achieve the execution of the centralized framework.

- CopyRemoteData (in remote_location, in data_handler, in local_repository): reads the data from the "remote location" based on the specifications of the data handler" and stores the data in the "local_repository"

- CombineData (in local_repository, out combined_data): combines the data read from the "local_repository" to create one large data source, "combined_data".

- FetchAlgorithm (in algorithm_location): fetches the data mining algorithm from the "algorithm location" and stores it locally for processing the data in the local repository

- ExecuteAlgorithm (in local_repository, out results): processes the data mining algorithm on the "local repository" with the logic specified in the algorithm. Returns the intermediate "results" for performing other tasks or the final "results". 


\subsubsection{DiSTRIBUTED SERVICES}

In the distributed approach, the datasets always reside on the remote sites and are never moved. The algorithm is propagated to the remote data sites where it processes the individual dataset and returns local models to a coordination site. The results are then processed to generate the global model and thereafter sent to the client. The number of intermediate results directly depends on the data mining algorithm itself.

- SendAlgorithm (in algorithm_location, in remote_locations): fetches the data mining algorithm from the "algorithm_location" and sends it to the "remotelocations" for processing the data.

- ExecuteAlgorithm (in remote_locations, out results): executes the algorithms concurrently on the "remote_locations" and stores the "results" on the remote location as the data model.

- CopyRemoteModel (in remote_models): reads the "remote_models" from the remote locations and copies it to the local coordination site for processing to get the final results.

The distributed approach may require multiple transfers of data between the coordination site and the data sites but it eventually proves to be the better of the two choices, especially for large real world data sets since the amount of data transferred is considerably less compared to the fetching of the actual datasets. The performance improvement can be measured in terms of execution time as well as the quality of service. 


\subsection{Selecting Dataset ANd Algorithm Service Information}

The first step in the exceution phase is to choose the desired datasets and the algorithm that can be applied on them. Figure 4.8 shows the steps involved in the selection of dataset and algorithm information from the registry.

The client uses the web interface to query the registry for information about available datasets based on classifications or keyword search. The registry returns the appropriate information on all the datasets that match the search criteria. Once the client selects the datasets for processing, a search for algorithms that match the selected datasets is performed and the algorithm service information that can be applied on them is displayed. This step of the implementation is common for the centralized and distributed approach.

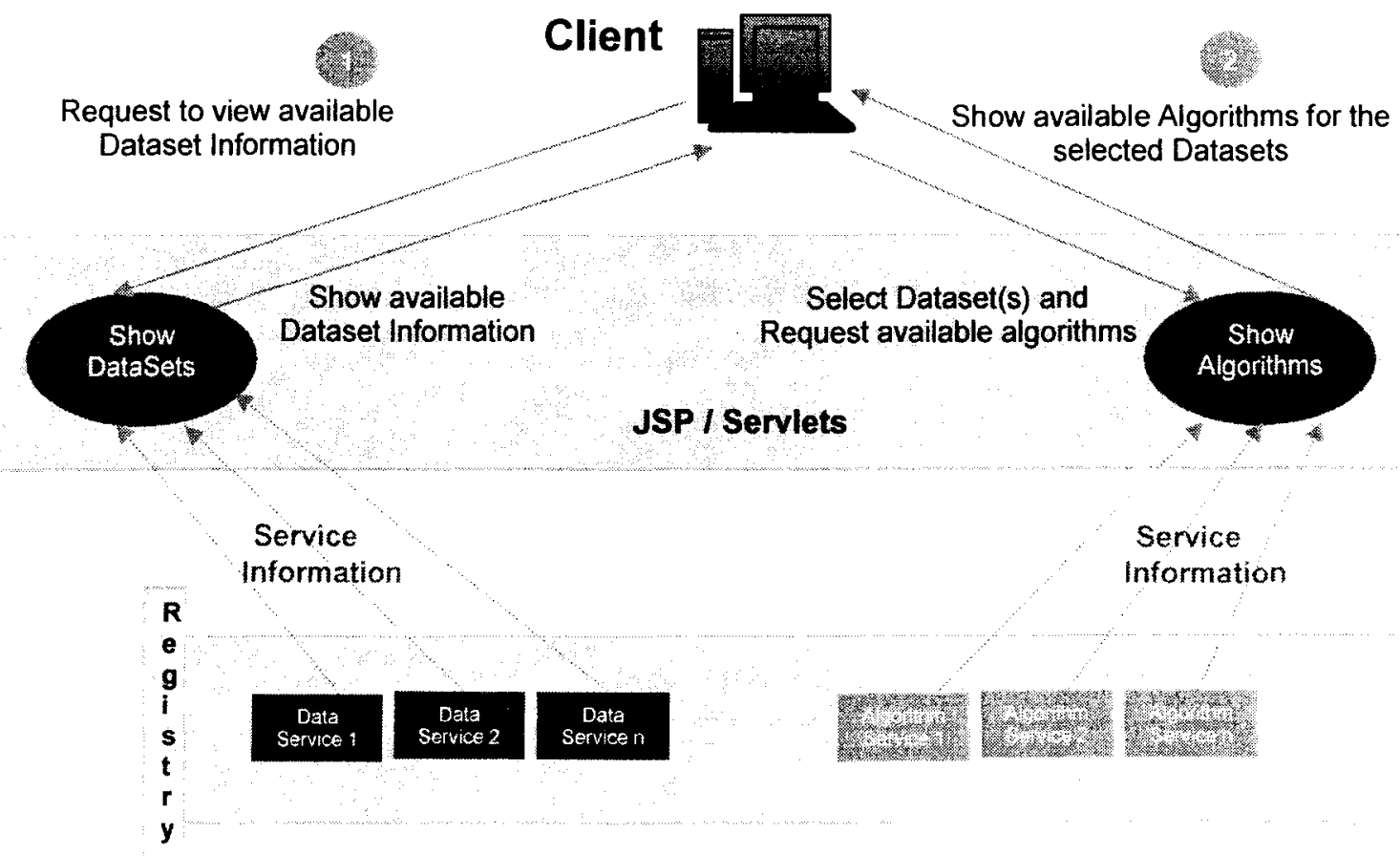

Figure 4.8: The client requests to view service information from the registry 


\subsubsection{EXeCUtion - Centralized APProaCh}

Step 1: The client invokes the data mining services from a URL. The client selects the required datasets located on remote sites.

Step 2: Servlet calls the service on the selected data sites to collect the data. The collected data is stored in a centralized location for processing as shown in Figure 4.9.

Step 3: The Apriori Service downloads the algorithm to the central site and is applied to build the global model for the datasets at the central site.

The datasets are now available as a single resource in the central site. The algorithm is applied on this combined data set and the results are returned to the client.

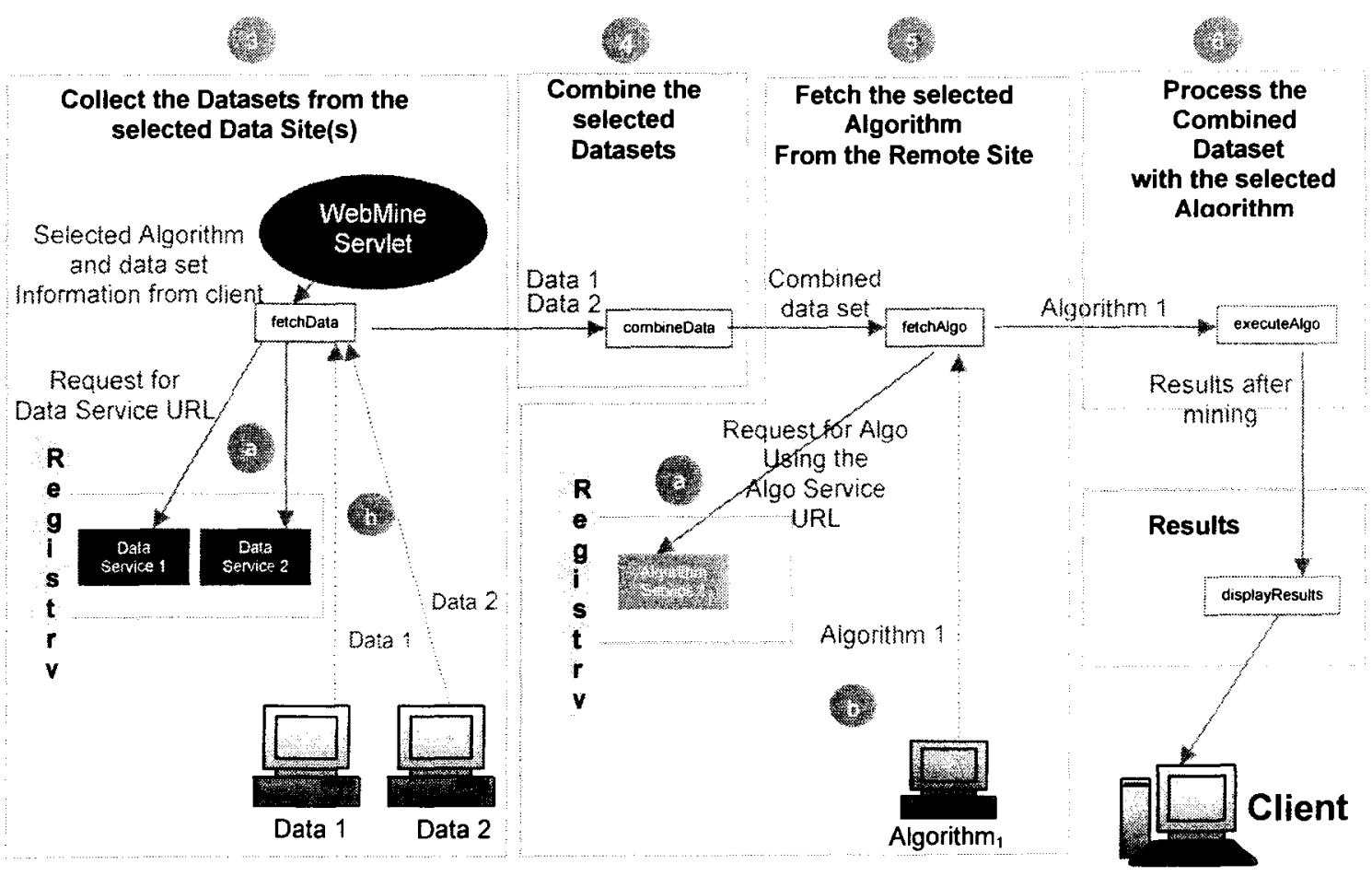

Figure 4.9: Client interaction in Centralized Approach 
$3 \mathrm{D}$, or in a higher dimensional space because only one dimension (one attribute) is assumed as independent variable and all others are dependent. Also, the dimensionality of rectangle and plane surface is always 2 regardless the dimensionality of the space for representation. These objects, representing an abstract relation in a data set, lead to the definition of the embedding and intrinsic dimensions of a data set [7]:

Definition 1 - The embedding dimension $E$ of a data set is the dimension of its address space. In other words, it is the number of attributes of the data set.

Definition 2 - The intrinsic dimension $D$ of a data set is the dimension of the spatial object represented by the data set, regardless of the space where it is embedded.

While the embedding dimension is given explicitly with the data set as the number of attributes, the intrinsic dimension is not computable directly. We can approximate it with the fractal dimension parameter $[7,16]$. The fractal dimension characterizes multidimensional fractal sets. By embedding the data set in an ndimensional grid with cell sides of size $r$, we can compute the frequency with which data points fall into the $i$-th cell, $p_{i}$. The generalized fractal dimension $D_{q}$ represents a derivative or linear slope of discrete frequency function, as shown in the equation:

$$
\mathrm{D}_{\mathrm{q}}=1 /(\mathrm{q}-1)\left[d\left(\log \Sigma_{\mathrm{i}} \mathrm{p}_{\mathrm{i}}^{\mathrm{q}}\right) / d(\log \mathrm{r})\right]
$$

Where:

$p_{i}$ - frequency of points falling in the i-th cell, and

r - size of the cell. 
There is a family of fractal dimensions (i.e. Hausdorff fractal dimension for $\mathrm{q}=0$, information fractal dimension for $\mathrm{q}=1$, and correlation fractal dimension for $\mathrm{q}=2$ ). Specifically, the correlation fractal dimension $(q=2)$ has gained attention in the literature

[7]. Changes in the correlation fractal dimension means changes in the distribution of points in the multidimensional data set, and that is the parameter we are using in the simple and scalable analysis of large, distributed data sets. As many real data sets are self-similar, we can use their correlation fractal dimension as a measure of their intrinsic dimension $D$. The correlation fractal dimension is usually calculated by means of the box-counting algorithm. Let $N(r)$ be $\Sigma_{i} p_{i}{ }^{2}$, then the plot of $N(r)$ for different values of $r$ in a log-log scale is called the box-counting plot. The linear slope of the plot is the estimation of the correlation fractal dimension for the given data set.

\subsection{APRIORI}

Association analysis identifies relationships or affinities between items and/or between features. These relationships are then expressed as a collection of association rules. The approach has been particularly successful in mining very large transaction databases and is one of the core classes of techniques in data mining.

Each transaction is thought of as a basket of items, which we might represent as $\{\mathrm{A}, \mathrm{B}, \mathrm{C}, \mathrm{D}, \mathrm{E}, \mathrm{F}\}$. The algorithm searches for collections of items that often appear together e.g. $\{\mathrm{A}, \mathrm{C}, \mathrm{F}\}$, and then from these itemsets it identifies rules like $\mathrm{A}, \mathrm{F} \rightarrow \mathrm{C}$ which we read as indicating an association between $\mathrm{A}$ and $\mathrm{F}$ being in the transaction and $\mathrm{C}$ consequently appearing in the transaction. 
The basis of an association analysis algorithm is the generation of frequent itemsets. However, naïve approaches will be quite expensive in computational time with even moderately sized databases. The apriori algorithm takes advantage of the simple apriori observation that all subsets of a frequent itemset must also be frequent. The observation allows the algorithm to consider a significantly reduced search space by starting with frequent individual items (eliminating rare items). We can then combine these into itemsets containing just two items and retain only those that are frequent enough. Similarly for itemsets containing three items, and so on.

Suppose we have a rule of the form $\mathrm{A} \rightarrow \mathrm{C}$. We call $\mathrm{A}$ the antecedent and $\mathrm{C}$ the consequent, and both are non-empty sets of items. The concept of frequent enough is a parameter of the algorithm, used to control the number of association rules discovered. This support specifies how frequently the items must appear in the whole data set before the items can be considered as a candidate association rule. For example, the user may choose to consider only sets of items that occur in at least $5 \%$ of all transactions. Formally we define support for a collection of items I as the proportion of all baskets in which all items in I appear. Then we can define the support for an association rule as:

$$
\text { support }(A \rightarrow C)=\operatorname{support}(A \cup C)
$$

A second parameter, the confidence, calculates the proportion of transactions containing $\mathrm{A}$ that also contain $\mathrm{C}$ the use specifies a minimal probability for the association rule. For example, the user may choose to only generate rules which are true at least $90 \%$ of the time (that is, when A appears in the basket, $\mathrm{C}$ also appears in the same basket at least $90 \%$ of the time). 
Formally:

$$
\text { confidence }(A \rightarrow C)=\operatorname{support}(A \rightarrow C) / \operatorname{support}(A)
$$

The Apriori algorithm is a breadth-first or generate-and-test type of search algorithm. Only after exploring all possibilities of associations containing $k$ items does it then consider those containing ${ }^{k+1}$ items. For each $k$, all candidates are tested to determine whether they have enough support. The apriori algorithm uses a simple two step generate and merge process: generate frequent itemsets of size $k$ then combine them to generate candidate frequent itemsets of size ${ }^{k+1}$. The algorithm is reasonably efficient even though the number of possible items is generally large and the baskets are generally small. The input data to the algorithm consists of records or transactions, each transaction representing a basket of items.

The two primary tuning parameters are minsup (minimum support expressed as a percentage of the total number of transactions in data) and mincon (minimum confidence also expressed as a percentage of the total number of transactions in data). Typically they have quite small values because of the size of the databases we are dealing with. Thus a support of $0.1 \%$ or smaller is not unusual. 


\section{RESULTS AND DISCUSSION}

In order to evaluate the effectiveness of the proposed service oriented framework three core data mining services including Normalization service (used by the Fractal Dimension algorithm), Fractal Dimension service and Apriori service were implemented. The services were implemented both in the centralized and distributed service oriented framework and their execution time was measured in a local and a wide area networks. The execution time for both implementations in a local area network is shown in the graphs included in this chapter. It is clear that as the number of records increase, the execution time of the centralized algorithm increases dramatically due to the overhead of combining all the data at the central site before performing the algorithm. The results illustrate that the distributed approach is more consistent and requires a shorter execution time. The performance results for the centralized and the distributed services in a wide area network are shown in various charts. It is interesting to observe that the increase in execution time for smaller number of records is much more significant here compared to the local area network. This shows the impact of additional communication delay when data sets are located on distant sites and are not on the same local area network. The distributed approach is consistent over time since it is dependent on the algorithms complexity rather than the dataset size. The apriori algorithm, whose complexity is higher than the fractal dimension algorithm showed similar results. 


\subsection{NORMALIZATION}

\section{Sample Input (Abridged)}

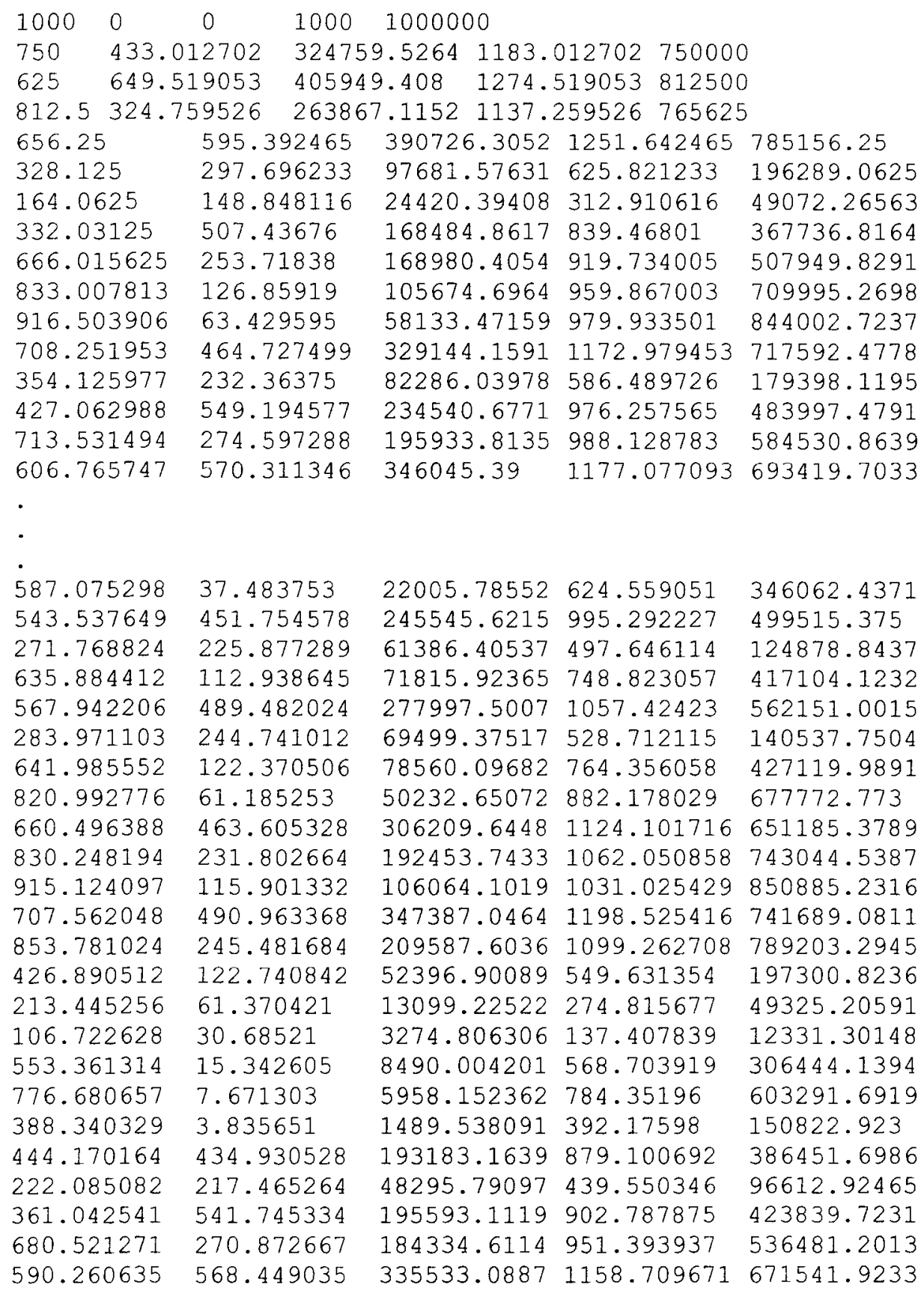

The sample input shown here is an abridged version. The actual datasets were distributed over the network on multiple data sites. The results obtained for a test run with data of the nature above, the following results were obtained. 


\section{Results (Abridged)}

Global Minimum and Maximum Values for each column

$\begin{array}{lllll}1.072523 & 0.0 & 0.0 & 1.40947 & 1.710467 \\ 1000.0 & 865.262736 & 432982.5973 & 1365.443297 & 1000000.0\end{array}$

Normalized Values

$$
\begin{aligned}
& 0.4107373542594024 \quad 0.5495562795160058 \quad 0.4517744510282653 \\
& 0.6491557866621734 \quad 0.39533401203878155 \\
& \begin{array}{llll}
0.20483183985938153 & 0.2747781397580029 & 0.11294361278016196
\end{array} \\
& 0.32406123825549377 \quad 0.09883222015225111 \\
& 0.6024159199296908 \quad 0.13738907045686063 \quad 0.16551352503977415 \\
& 0.5280737887448299 \quad 0.37754971381932634 \\
& 0.3006711226945257 \quad 0.06869453465057115 \quad 0.04137838125994354 \\
& 0.2635202389302622 \quad 0.0943861455973873 \\
& \begin{array}{llll}
0.6503355618477997 & 0.03434726790314474 & 0.044664000771838866
\end{array} \\
& 0.4978032887156543 \quad 0.4243065234953063 \\
& \begin{array}{llll}
0.32463094315304336 & 0.017173633373713207 & 0.011166000195269279
\end{array} \\
& 0.2483849896487941 \quad 0.10607534797138221 \\
& 0.161778634306202 \quad 0.008586817264715765 \quad 0.002791500047662539 \\
& 0.12367583974880411 \quad 0.026517554150401298 \\
& \begin{array}{lll}
0.08035247988278132 & 0.004293408054498721 & 6.978750113382443 \mathrm{E}-4
\end{array} \\
& 0.061321264432249306 \quad 0.006628105685156048 \\
& 0.03963940317160783 \quad 0.0021467040272493605 \quad 1.744687534119515 \mathrm{E}-4 \\
& 0.030143977507091545 \quad 0.0016557435690947352 \\
& \text {. } \\
& \dot{0} .8536240324081105 \quad 0.2837076806691465 \quad 0.48405549069858655 \\
& 0.8048577801142757 \quad 0.7892029339385748 \\
& 0.42627517893373557 \quad 0.14185384033457324 \quad 0.12101387265155102 \\
& 0.4019122349815449 \quad 0.1972994506071994 \\
& \begin{array}{lll}
0.21260075219654812 & 0.07092692016728662 & 0.030253468157113852
\end{array} \\
& 0.20043946241517954 \quad 0.04932357980935559 \\
& \begin{array}{llll}
0.10576353882795438 & 0.035463459505784146 & 0.007563367041588024
\end{array} \\
& 0.09970307649855666 \quad 0.012329612102394624 \\
& 0.5528817694139772 \quad 0.017731729752892073 \quad 0.01960818807486053 \\
& 0.4158947071332418 \quad 0.3064429530935586 \\
& 0.7764408847069886 \quad 0.0088658654543052 \quad 0.013760720174792114 \\
& 0.5739905231837039 \quad 0.6032910133423697 \\
& \begin{array}{llll}
0.3876836055837115 & 0.004432932149293437 & 0.003440180044852809
\end{array} \\
& 0.28647860651625906 \quad 0.1508214705081482 \\
& \begin{array}{llll}
0.44357338365615906 & 0.5026571813442802 & 0.4461684259474971
\end{array} \\
& 0.6434526802977885 \quad 0.3864506491440825 \\
& 0.22124985455775986 \quad 0.2513285906721401 \quad 0.11154210647532646 \\
& 0.32120968507330133 \quad 0.09661137943357635 \\
& \begin{array}{ccc}
0.36035650864372015 & 0.6261050100278442 & 0.4517343494165417 \\
0.6608182195763096 & 0.423838737595174 & \\
0.6801782548223968 & 0.3130525050139221 & 0.4257321484731183
\end{array} \\
& \begin{array}{ccc}
0.6801782548223968 & 0.3130525050139221 \\
0.6964522786721183 & 0.5364804084650348
\end{array} \\
& \begin{array}{lll}
0.5898207082755017 & 0.6569669666208762 & 0.7749343525405472
\end{array} \\
& 0.848439516742278 \quad 0.671541361482338
\end{aligned}
$$


Table 6.1 presents a summary of the total execution time for various sizes of datasets in a Local Area Network and Figure 6.1 shows the difference in the execution times for both the approaches.

\begin{tabular}{|r|r|r|}
\hline $\begin{array}{r}\text { Number of } \\
\text { Records }\end{array}$ & $\begin{array}{r}\text { Centralized } \\
\text { Approach (in ms) }\end{array}$ & $\begin{array}{r}\text { Distributed } \\
\text { Approach (in ms) }\end{array}$ \\
\hline 10 & 2 & 10093 \\
\hline 100 & 953 & 10572 \\
\hline 1000 & 1584 & 11174 \\
\hline 10000 & 11280 & 12045 \\
\hline 25000 & 19985 & 12986 \\
\hline 50000 & 41063 & 14193 \\
\hline
\end{tabular}

Table 6.1: Execution Times for the Normalization algorithm in a Local Area Network

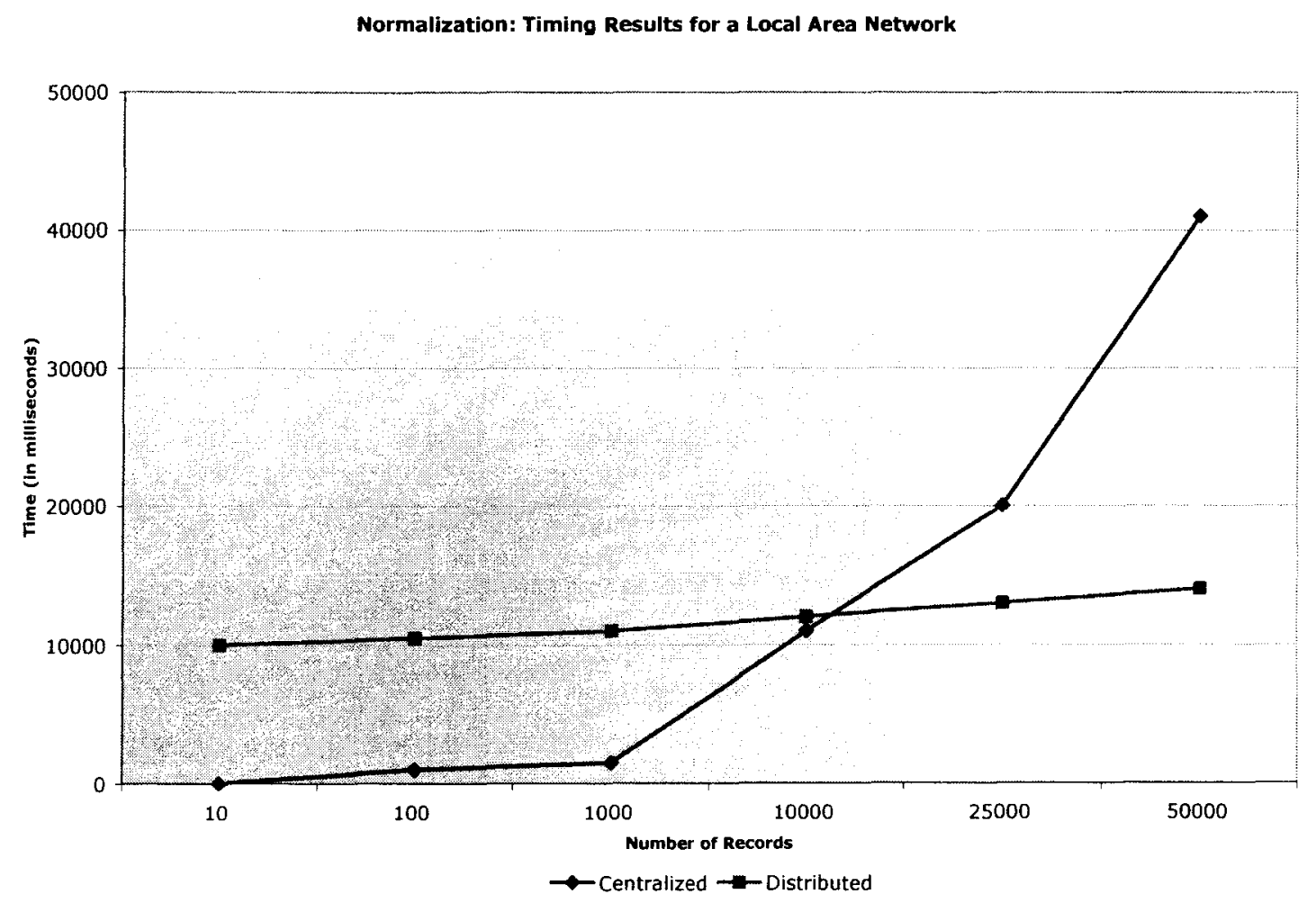

Figure 6.1: Timing Results the Normalization algorithm in a Local Area Network 
Table 6.2 presents a summary of the total execution time for various sizes of datasets in a Wide Area Network and Figure 6.2 shows the difference in the execution times for both the approaches.

\begin{tabular}{|r|r|r|}
\hline $\begin{array}{r}\text { Number of } \\
\text { Records }\end{array}$ & $\begin{array}{r}\text { Centralized } \\
\text { Approach (in ms) }\end{array}$ & $\begin{array}{r}\text { Distributed } \\
\text { Approach (in ms) }\end{array}$ \\
\hline 10 & 3 & 20675 \\
\hline 100 & 1121 & 22128 \\
\hline 1000 & 14752 & 23284 \\
\hline 10000 & 58163 & 24117 \\
\hline 25000 & 70034 & 25283 \\
\hline 50000 & 95178 & 26131 \\
\hline
\end{tabular}

Table 6.2: Execution Times for the Normalization algorithm in a Wide Area Network Normalization: Timing Results for a Wide Area Network

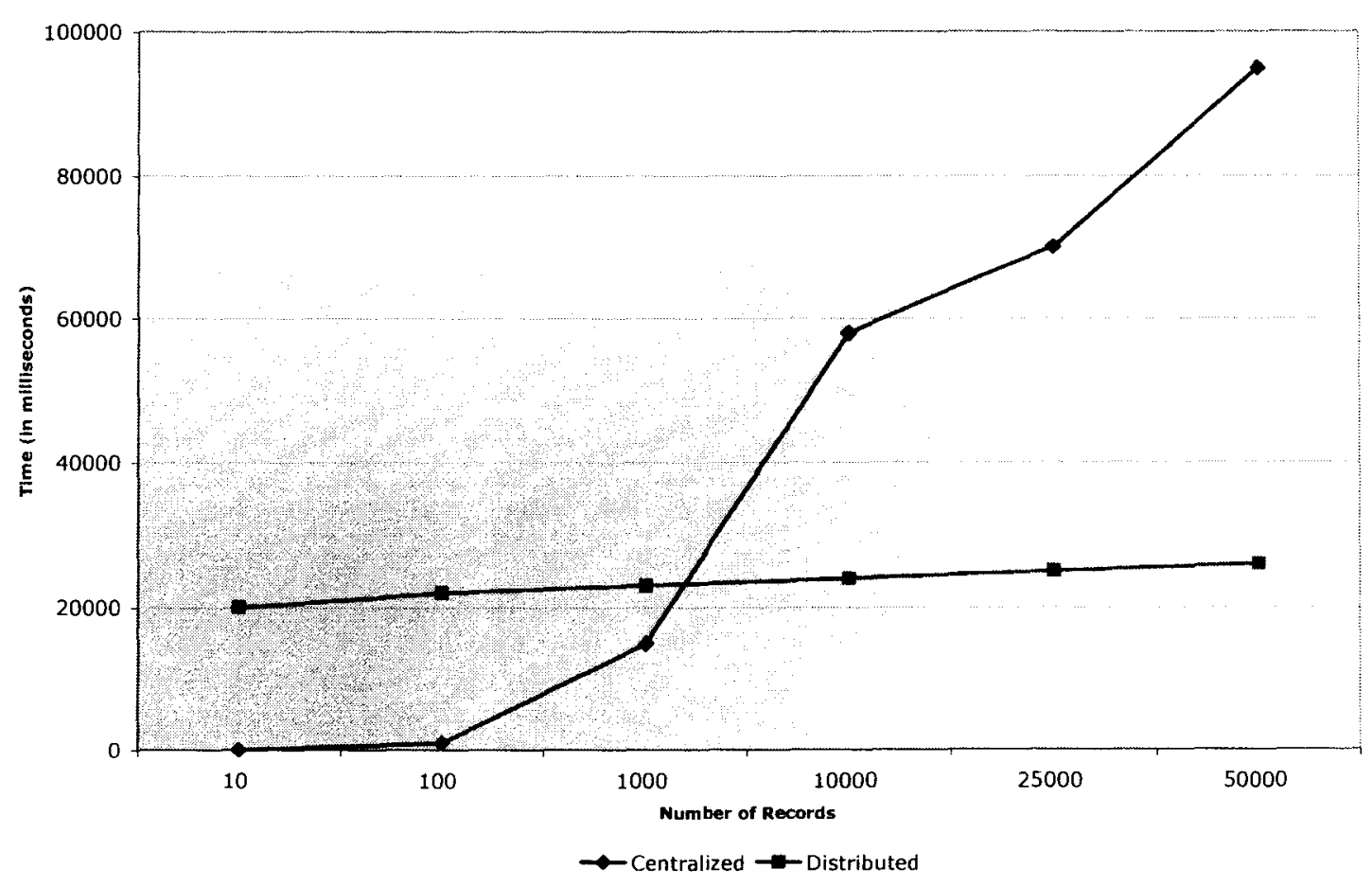

Figure 6.2: Timing Results the Normalization algorithm in a Wide Area Network 


\subsection{Fractal Dimension}

\section{Sample Input (Abridged)}

\begin{tabular}{|c|c|c|c|c|}
\hline 4 & 162 & 28 & 13 & \\
\hline 739706064 & 0.864580806 & 0.259423693 & 0.011418937 & 0.356706385 \\
\hline 0.424688613 & 0.078193912 & 0.153734069 & 0.033618253 & 0.376185509 \\
\hline 0.729208003 & 0.763120257 & 0.81536836 & 0.943994791 & 0.184170159 \\
\hline 0.94646033 & 0.398229453 & 0.222784329 & 0.176225524 & 0.922198721 \\
\hline 0.124967392 & 0.516087951 & 0.711096562 & 0.890662873 & 0.411399253 \\
\hline 0.563390336 & 0.267818823 & 0.459989708 & 0.77024769 & 0.813885662 \\
\hline 0.382414158 & 0.900767957 & 0.653436678 & 0.612937901 & 0.874448675 \\
\hline 0.171765921 & 0.124435936 & 0.296652074 & 0.631348493 & 0.684876036 \\
\hline 0.378876905 & 0.161410775 & 0.42654536 & 0.313310403 & 0.232790797 \\
\hline 0.449745042 & 0.157385243 & 0.891801018 & 0.589122929 & 0.987613927 \\
\hline 0.567700996 & 0.602806213 & 0.371143355 & 0.21021309 & 0.714086604 \\
\hline 0.255867668 & 0.087695473 & 0.468571623 & 0.053235229 & 0.034510664 \\
\hline 0.14056215 & 0.672202741 & 0.914448435 & 0.009408939 & 0.616517722 \\
\hline 0.031873775 & 0.243668039 & 0.275138193 & 0.343516135 & 287067 \\
\hline 0.97361543 & 0.088462958 & 0.006037269 & 0.503342929 & 236925 \\
\hline 0.520164863 & 0.750445934 & 0.340843093 & 0.631344558 & 32528 \\
\hline 0.860985541 & 0.950320833 & 0.312228982 & 0.612159987 & 0.234560139 \\
\hline & & & & \\
\hline & & & & \\
\hline 05 & 72512484 & 221 & 0.24 & 0 \\
\hline 5194 & 0.827046468 & 0.634690871 & 0.5103693 & 0.5 \\
\hline .315227562 & 0.061213551 & 0.389608188 & 0.553344671 & 0.60934108 \\
\hline 0.55011439 & 0.884756061 & 0.40060435 & 0.546643909 & 0.80115288 \\
\hline 0.333780101 & 0.265699934 & 0.650382835 & 0.621150656 & 0.53191915 \\
\hline .189299685 & 0.323530236 & 0.601776414 & 0.257489409 & 0.01481741 \\
\hline 0.109534611 & 0.950736971 & 0.399120502 & 0.973776989 & 0.67513459 \\
\hline 0.708144024 & 0.893788511 & 0.10829746 & 0.800678545 & 0.67531541 \\
\hline 0.48400863 & 0.660084574 & 0.901932356 & 0.088998619 & 0.26676421 \\
\hline 0.102632789 & 0.167947575 & 0.624461557 & 0.048280511 & 0.37422655 \\
\hline 0.4903537 & 0.975016942 & 0.852711187 & 0.687892242 & 0.00103883 \\
\hline 98228721 & 0.254021122 & 0.952769735 & 0.362897777 & 0.23039182 \\
\hline 889392779 & 0.937807216 & 0.415990575 & 0.654767107 & 0.6790892 \\
\hline 47029756 & 590564596 & 0.146221548 & 0.253154055 & 1 \\
\hline 37302612 & 960276301 & 0.08488191 & 0.83 & $6683^{7}-x$ \\
\hline 4236786 & 0.550800623 & 0.62 & 440590.91 & 27382 \\
\hline $2 J$ & 0 & 52570. & 260110. & 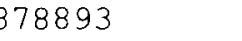 \\
\hline 0.20 & $0063 \quad 0.323$ & 810510.08 & $27406 \quad 0.6$ & 1264 \\
\hline 6 & 1. & .615742464 & 1 & 007 \\
\hline
\end{tabular}

The sample input shown here is an abridged version. The actual datasets were distributed over the network on multiple data sites. The results obtained for a test run with data of the nature above, the following results were obtained. 


\section{Results (Abridged)}

\begin{tabular}{|c|c|c|c|c|c|c|c|}
\hline$r=1 / 2$ & & $r=1 / 4$ & & $r=1 / 8$ & & $r=1 / 16$ & \\
\hline 1.1 .1 .1 .1 & 285 & 1.1 .1 .1 .1 & 5 & 1.1 .1 .1 .3 & 2 & 1.1 .1 .4 .12 & 1 \\
\hline 1.1 .1 .1 .2 & 313 & 1.1 .1 .1 .2 & 10 & 1.1 .1 .1 .5 & 1 & 1.1 .1 .8 .10 & 1 \\
\hline 1.1 .1 .2 .1 & 339 & 1.1 .1 .1 .3 & 12 & 1.1 .1 .2 .1 & 1 & 1.1 .1 .9 .10 & 1 \\
\hline 1.1 .1 .2 .2 & 314 & 1.1 .1 .1 .4 & 9 & 1.1 .1 .2 .6 & 1 & 1.1 .10 .13 .4 & 1 \\
\hline $\begin{array}{l}1.1 .2 .1 .1 \\
\cdot \\
\cdot \\
.\end{array}$ & 306 & $\begin{array}{l}1.1 \cdot 1 \cdot 2 \cdot 1 \\
\cdot \\
\cdot\end{array}$ & 13 & $\begin{array}{l}1.1 .1 .4 .2 \\
. \\
.\end{array}$ & 1 & $\begin{array}{l}1.1 .10 \cdot 3.4 \\
. \\
\cdot\end{array}$ & 1 \\
\hline 2.2 .1 .2 .2 & 323 & 1.1 .2 .1 .3 & 10 & 8.8 .8 .7 .8 & 1 & 9.9 .8 .5 .11 & 1 \\
\hline 2.2 .2 .1 .1 & 341 & 1.1 .2 .1 .4 & 6 & 8.8 .8 .8 .2 & 2 & 9.9 .8 .7 .10 & 1 \\
\hline 2.2 .2 .1 .2 & 303 & 1.1 .2 .2 .1 & 7 & 8.8 .8 .8 .6 & 1 & 9.9 .8 .7 .15 & 1 \\
\hline 2.2 .2 .2 .1 & 317 & $1.1 \cdot 2 \cdot 2 \cdot 2$ & 6 & 8.8 .8 .8 .7 & 1 & $9 \cdot 9 \cdot 9 \cdot 15.10$ & 1 \\
\hline 2.2 .2 .2 .2 & 324 & 1.1 .2 .2 .3 & 11 & 8.8 .8 .8 .8 & 1 & 9.9 .9 .4 .2 & 1 \\
\hline
\end{tabular}

\begin{tabular}{|ll|ll|ll|}
\hline$r=1 / 32$ & & $r=1 / 64$ & & $r=1 / 128$ & \\
1.1 .26 .31 .24 & 1 & 1.10 .13 .13 .31 & 1 & 1.103 .51 .112 .13 & 1 \\
1.1 .28 .3 .24 & 1 & 1.10 .39 .45 .3 & 1 & 1.104 .115 .60 .44 & 1 \\
1.1 .6 .28 .10 & 1 & 1.10 .52 .52 .24 & 1 & 1.104 .121 .123 .18 & 1 \\
1.10 .13 .30 .10 & 1 & 1.10 .9 .41 .21 & 1 & 1.105 .26 .21 .119 & 1 \\
1.10 .19 .29 .5 & 1 & 1.11 .25 .19 .5 & 1 & 1.105 .31 .32 .87 & 1 \\
$\cdot$ & & & & $\cdot$ & \\
$\cdot$ & & & & $\cdot$ & \\
9.9 .25 .2 .3 & 1 & 9.64 .6 .20 .48 & 1 & 99.86 .24 .87 .66 & 1 \\
9.9 .29 .8 .10 & 1 & 9.7 .11 .49 .45 & 1 & 99.88 .62 .13 .61 & 1 \\
9.9 .4 .25 .16 & 1 & 9.7 .11 .63 .45 & 1 & 99.89 .54 .7 .38 & 1 \\
9.9 .7 .29 .18 & 1 & 9.8 .30 .48 .19 & 1 & 99.9 .102 .77 .9 & 1 \\
9.9 .9 .30 .28 & 1 & 9.9 .5 .23 .55 & 1 & 99.97 .94 .103 .11 & 1 \\
& & & & & \\
\hline
\end{tabular}

$F D=2.788957407590457$

$R=0.9404928239071236$

$\mathrm{FD}=1.0678309573125557$

$R=0.8293589297488905$

$F D=0.11581529365873741$

$R=0.7949366774769094$

$F D=0.004621321783703003$

$R=0.793912851301406$

The FD: 2.788957407590457

The R: 0.9404928239071236 
Table 6.3 presents a summary of the total execution time for various sizes of datasets in a Local Area Network and Figure 6.3 shows the difference in the execution times for both the approaches.

\begin{tabular}{|r|r|r|}
\hline $\begin{array}{r}\text { Number of } \\
\text { Records }\end{array}$ & $\begin{array}{r}\text { Centralized } \\
\text { Approach (in ms) }\end{array}$ & $\begin{array}{r}\text { Distributed } \\
\text { Approach (in ms) }\end{array}$ \\
\hline 10 & 28 & 20048 \\
\hline 100 & 752 & 21613 \\
\hline 1000 & 1759 & 21934 \\
\hline 10000 & 58125 & 25912 \\
\hline 20000 & 80474 & 30945 \\
\hline 30000 & 92087 & 33117 \\
\hline 40000 & 112790 & 42283 \\
\hline 50000 & 146183 & 51743 \\
\hline 60000 & 165251 & 56276 \\
\hline
\end{tabular}

Table 6.3: Execution Times for the Fractal Dimension algorithm in a Local Area Network

Fractal Dimension: Tìming Results for a Local Area Network

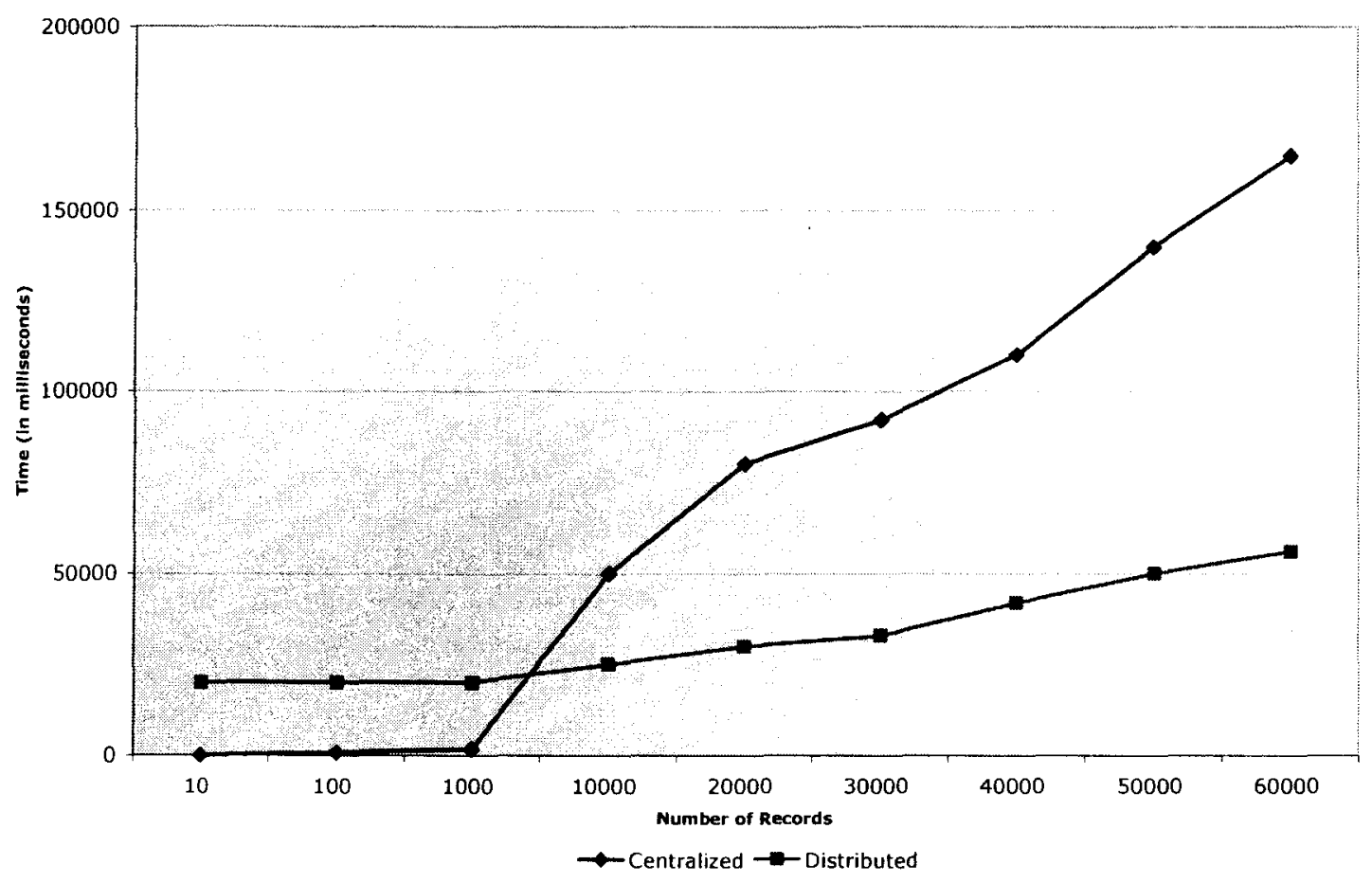

Figure 6.3: Timing Results the Fractal Dimension algorithm in a Local Area Network 
Table 6.4 presents a summary of the total execution time for various sizes of datasets in a Wide Area Network and Figure 6.4 shows the difference in the execution times for both the approaches.

\begin{tabular}{|r|r|r|}
\hline $\begin{array}{r}\text { Number of } \\
\text { Records }\end{array}$ & $\begin{array}{r}\text { Centralized } \\
\text { Approach (in ms) }\end{array}$ & $\begin{array}{r}\text { Distributed } \\
\text { Approach (in ms) }\end{array}$ \\
\hline 10 & 27 & 103425 \\
\hline 100 & 7512 & 121533 \\
\hline 1000 & 17509 & 177342 \\
\hline 10000 & 165433 & 185630 \\
\hline 20000 & 189342 & 192153 \\
\hline 30000 & 240342 & 213436 \\
\hline 40000 & 453262 & 235121 \\
\hline 50000 & 648211 & 247345 \\
\hline 60000 & 812424 & 245841 \\
\hline
\end{tabular}

Table 6.4: Execution Times for the Fractal Dimension algorithm in a Wide Area Network

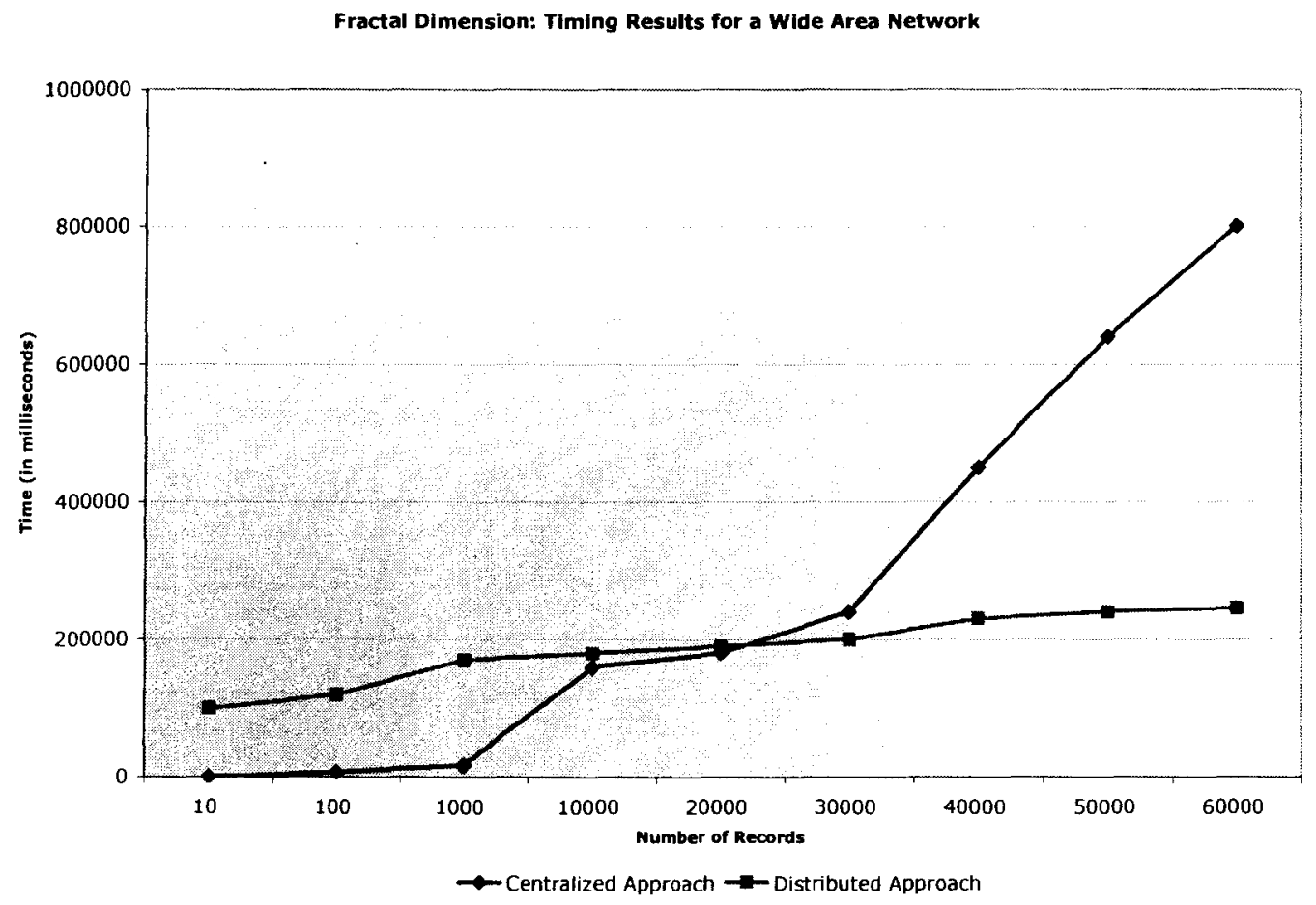

Figure 6.4: Timing Results the Fractal Dimension algorithm in a Wide Area Network 


\subsection{APRIORI}

\section{Sample Input (For generating Maximal Frequent Itemsets)}

\begin{tabular}{|c|c|}
\hline Site 1 & Site 2 \\
\hline $\begin{array}{llll}1 & 3 & 4 & \\
2 & 3 & 5 & \\
1 & 2 & 3 & 5 \\
2 & 5 & & \end{array}$ & $\begin{array}{llll}1 & 2 & 3 & 4 \\
2 & 3 & 5 & \\
1 & 2 & 3 & 5 \\
2 & 5 & & \\
3 & 4 & 5 & \\
1 & 2 & 4 & \\
1 & 3 & & \end{array}$ \\
\hline
\end{tabular}

\section{Results (Maximal Frequent Itemsets)}

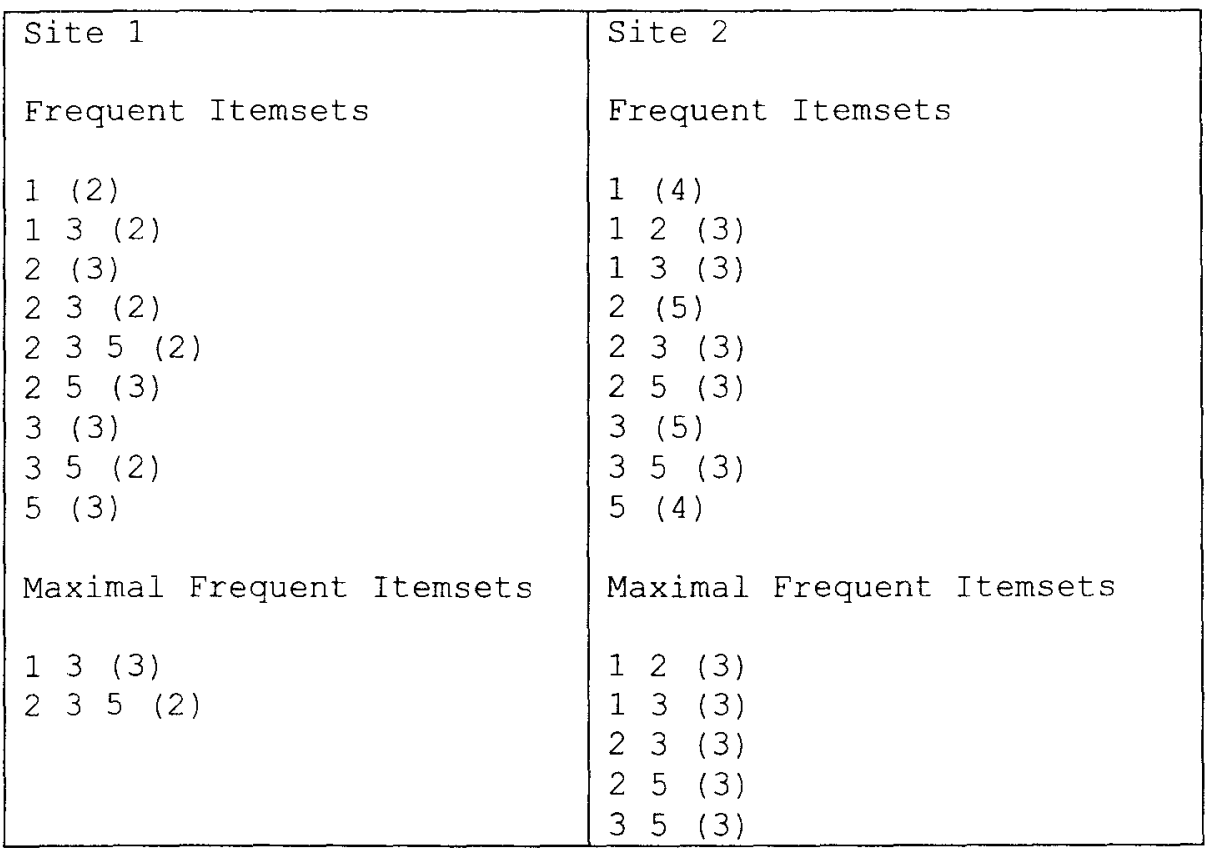

\section{Similarity Matrix Generation}

\section{Iteration 1 (FI 1 to FI 2)}

The first element $\left\{\begin{array}{ll}1 & 3\end{array}\right\}$ from FI 1 is searched in FI 2. The second element is a perfect match. Hence we consider the existence of $\{13\}$ as a value of 1 .

$\left\{\begin{array}{ll}1 & 3\end{array}\right\} \leftrightarrow\left\{\begin{array}{ll}1 & 3\end{array}\right\} \rightarrow 1$

The second element $\left\{\begin{array}{lll}2 & 3 & 5\end{array}\right\}$ from FI 1 is searched in FI 2. 
$\left\{\begin{array}{ll}235 \\ 2\end{array} \rightarrow\left\{\begin{array}{lll}2 & 3 & 5\end{array}\right\} \rightarrow 0\right.$

Since there exists no perfect match, a subset search is initiated. The subsets are created in a tree fashion to enable search based on the size of the subset. The results of the subset search yield the following values:

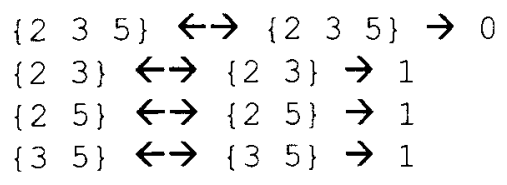

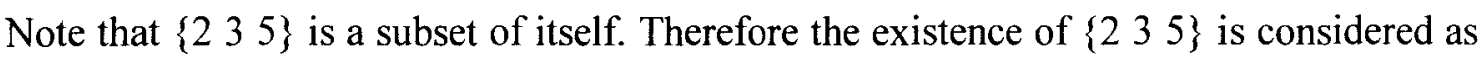
$3 / 4$ since one of the four subsets failed to match. The similarity matrix entry for $S_{1} S_{2}$ is calculated as $(1+3 / 4) / 2=0.875$.

\begin{tabular}{|l|l|l|l|}
\hline \multicolumn{3}{|c|}{ To } \\
\hline \multirow{3}{*}{ From } & & $\mathrm{S}_{1}$ & $\mathrm{~S}_{2}$ \\
\cline { 2 - 4 } & $\mathrm{S}_{1}$ & 1 & 0.875 \\
\cline { 2 - 4 } & $\mathrm{S}_{2}$ & & 1 \\
\hline
\end{tabular}

\section{Iteration 1 (FI 2 to FI 1)}

The first element $\{12\}$ from FI 2 is searched in FI 1.

$\left\{\begin{array}{ll}1 & 2\}\end{array} \rightarrow\left\{\begin{array}{ll}1 & 2\end{array}\right\} \rightarrow 0\right.$

The second element $\{13\}$ from FI 2 is searched in FI 1.

$\{13\} \leftrightarrow\left\{\begin{array}{lll}1 & 3\end{array}\right\} \rightarrow 1$

The third element $\{23\}$ from FI 2 is searched in FI 1.

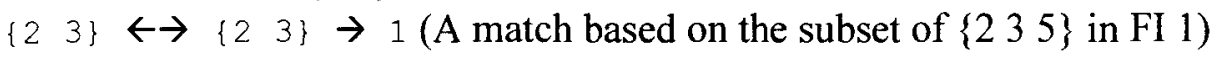

The fourth element $\{25\}$ from FI 2 is searched in FI 1.

$\{25\} \leftrightarrow\{25\} \rightarrow 1$ (A match based on the subset of $\{235\}$ in FI 1)

The fifth element $\{35\}$ from FI 2 is searched in FI 1.

$\{35\} \leftrightarrow\{35\} \rightarrow 1$ (A match based on the subset of $\{235\}$ in FI 1)

The similarity matrix entry for $S_{2} S_{1}$ is calculated as $(1+1+1+1) / 5=0.8$

\begin{tabular}{|l|l|l|l|}
\hline \multicolumn{4}{|c|}{ To } \\
\hline \multirow{3}{*}{ From } & & $\mathrm{S}_{1}$ & $\mathrm{~S}_{2}$ \\
\cline { 2 - 4 } & $\mathrm{S}_{1}$ & 1 & 0.875 \\
\cline { 2 - 4 } & $\mathrm{S}_{2}$ & 0.8 & 1 \\
\hline
\end{tabular}


Table 6.5 presents a summary of the total execution time for various sizes of datasets distributed over several sites and the algorithm execution performed using the centralized approach.

\begin{tabular}{|l|l|l|l|l|l|}
\hline $\begin{array}{l}\text { Number } \\
\text { of Sites }\end{array}$ & $\begin{array}{c}10,000 \\
\text { Records }\end{array}$ & $\begin{array}{c}25,000 \\
\text { Records }\end{array}$ & $\begin{array}{l}50,000 \\
\text { Records }\end{array}$ & $\begin{array}{l}75,000 \\
\text { Records }\end{array}$ & $\begin{array}{l}\mathbf{1 0 0 , 0 0 0} \\
\text { Records }\end{array}$ \\
\hline $\mathbf{2}$ & 18,334 & 27,453 & 49,565 & 81,532 & 105,648 \\
\hline 3 & 26,321 & 40,453 & 67,453 & 106,732 & 126,321 \\
\hline 4 & 31,743 & 58,454 & 91,343 & 117,321 & 156,896 \\
\hline 5 & 44,982 & 71,454 & 100,565 & 149,697 & 178,564 \\
\hline 6 & 53,683 & 89,454 & 121,538 & 163,998 & 184,232 \\
\hline 7 & 70,564 & 110,768 & 149,576 & 189,432 & 197,546 \\
\hline
\end{tabular}

Table 6.5: Execution Times in Milliseconds for the Centralized approach

The results were plotted to measure performance changes as well as to determine the quality of the results. Figure 6.5 shows the centralized approach implementation of the Apriori algorithm.

\section{CENTRALIZED APPROACH}

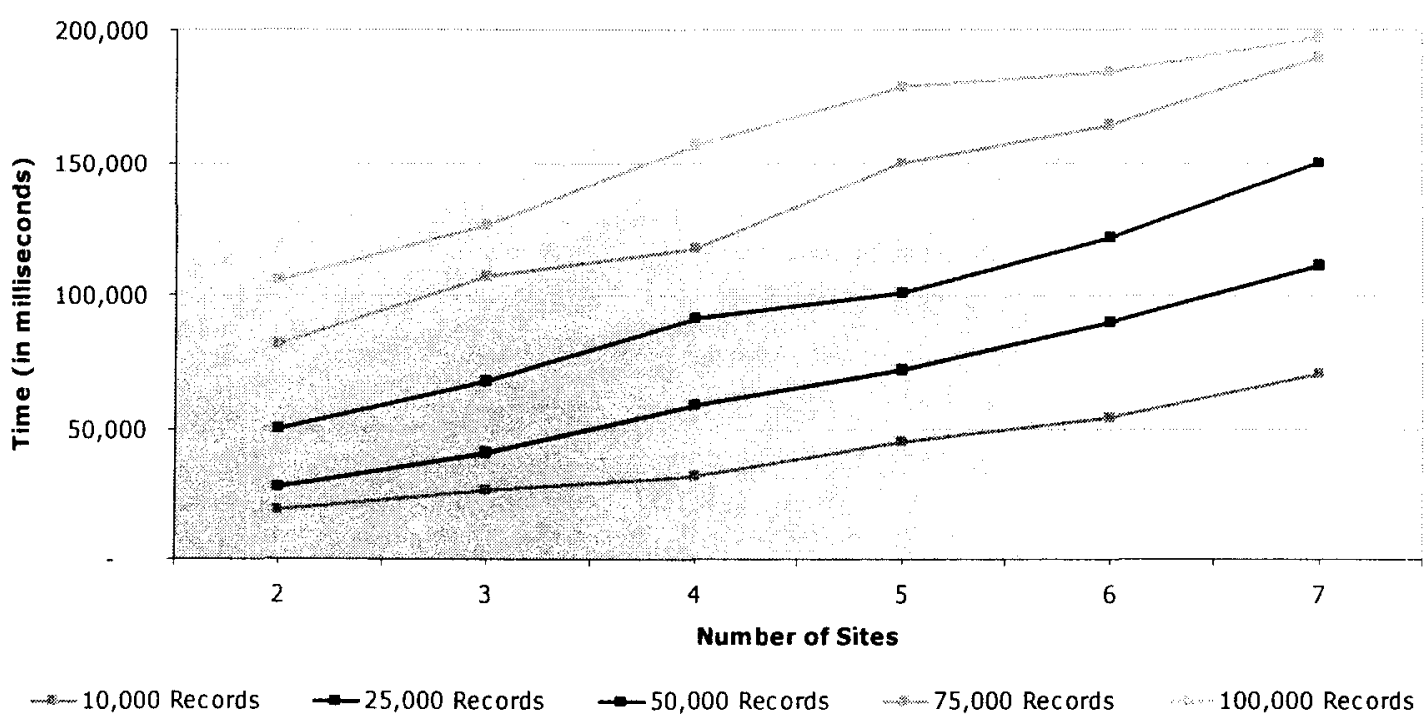

Figure 6.5: Timing Results for the Centralized approach 
Table 6.6 presents a summary of the total execution time for various sizes of datasets distributed over several sites and the algorithm execution performed using the distributed approach.

\begin{tabular}{|r|l|l|l|l|l|}
\hline $\begin{array}{c}\text { Number } \\
\text { of Sites }\end{array}$ & $\begin{array}{c}\mathbf{1 0 , 0 0 0} \\
\text { Records }\end{array}$ & $\begin{array}{c}\mathbf{2 5 , 0 0 0} \\
\text { Records }\end{array}$ & $\begin{array}{l}\mathbf{5 0 , 0 0 0} \\
\text { Records }\end{array}$ & $\begin{array}{l}\mathbf{7 5 , 0 0 0} \\
\text { Records }\end{array}$ & $\begin{array}{l}\mathbf{1 0 0 , 0 0 0} \\
\text { Records }\end{array}$ \\
\hline $\mathbf{2}$ & 29,528 & 33,632 & 40,985 & 48,345 & 57,345 \\
\hline $\mathbf{3}$ & 32,743 & 37,458 & 47,684 & 53,896 & 60,684 \\
\hline $\mathbf{4}$ & 34,743 & 40,975 & 51,564 & 60,657 & 69,489 \\
\hline $\mathbf{5}$ & 36,965 & 44,953 & 55,907 & 67,343 & 76,984 \\
\hline $\mathbf{6}$ & 39,564 & 47,932 & 59,567 & 71,569 & 83,673 \\
\hline $\mathbf{7}$ & 43,742 & 50,785 & 61,843 & 75,311 & 89,565 \\
\hline
\end{tabular}

Table 6.6: Execution Times in Milliseconds for the Distributed approach

The results were plotted to measure performance changes as well as to determine the quality of the results. Figure $\mathbf{6 . 6}$ shows the distributed approach implementation of the Apriori algorithm.

DISTRIBUTED APPROACH

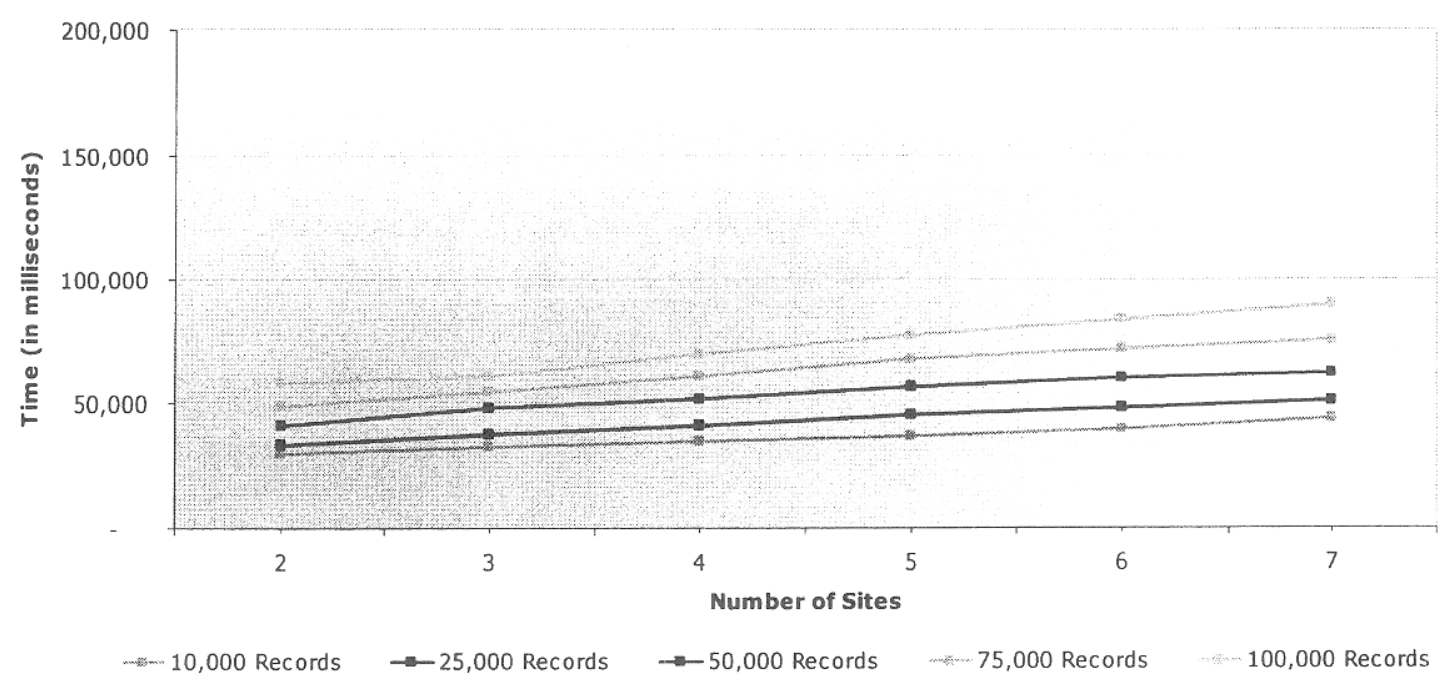

Figure 6.6: Timing Results for the Distributed approach 
It is clear that as the number of records increase, the execution time of the centralized algorithm increases dramatically due to the overhead of combining all the data at the central site before executing the algorithm. Table 6.7 shows the results obtained when a comparison of both the approaches was performed. The results illustrate that the distributed approach is more consistent and requires a shorter execution time in the long run.

\begin{tabular}{|r|r|r|r|r|r|}
\hline $\begin{array}{r}\text { Number } \\
\text { of Sites }\end{array}$ & $\begin{array}{c}1 \mathbf{1 0 , 0 0 0} \\
\text { Records }\end{array}$ & $\begin{array}{c}\mathbf{2 5 , 0 0 0} \\
\text { Records }\end{array}$ & $\begin{array}{r}\mathbf{5 0 , 0 0 0} \\
\text { Records }\end{array}$ & $\begin{array}{r}\mathbf{7 5 , 0 0 0} \\
\text { Records }\end{array}$ & $\begin{array}{r}\mathbf{1 0 0 , 0 0 0} \\
\text { Records }\end{array}$ \\
\hline $\mathbf{2}$ & $-61.1 \%$ & $-22.5 \%$ & $17.3 \%$ & $40.7 \%$ & $45.7 \%$ \\
\hline $\mathbf{3}$ & $-24.4 \%$ & $7.4 \%$ & $29.3 \%$ & $49.5 \%$ & $52.0 \%$ \\
\hline $\mathbf{4}$ & $-9.5 \%$ & $29.9 \%$ & $43.5 \%$ & $48.3 \%$ & $55.7 \%$ \\
\hline $\mathbf{5}$ & $17.8 \%$ & $37.1 \%$ & $44.4 \%$ & $55.0 \%$ & $56.9 \%$ \\
\hline $\mathbf{6}$ & $26,3 \%$ & $46.4 \%$ & $51.0 \%$ & $56.4 \%$ & $54.6 \%$ \\
\hline $\mathbf{7}$ & $38.0 \%$ & $54.2 \%$ & $58.7 \%$ & $60.2 \%$ & $54.7 \%$ \\
\hline
\end{tabular}

Table 6.7: Percentage Improvement of Distributed approach Vs Centralized approach

The percentage of improvement of the distributed framework over the centralized framework is shown in Figure 6.7.

Percentage Improvement of Distributed Approach vs. Centralized Approach

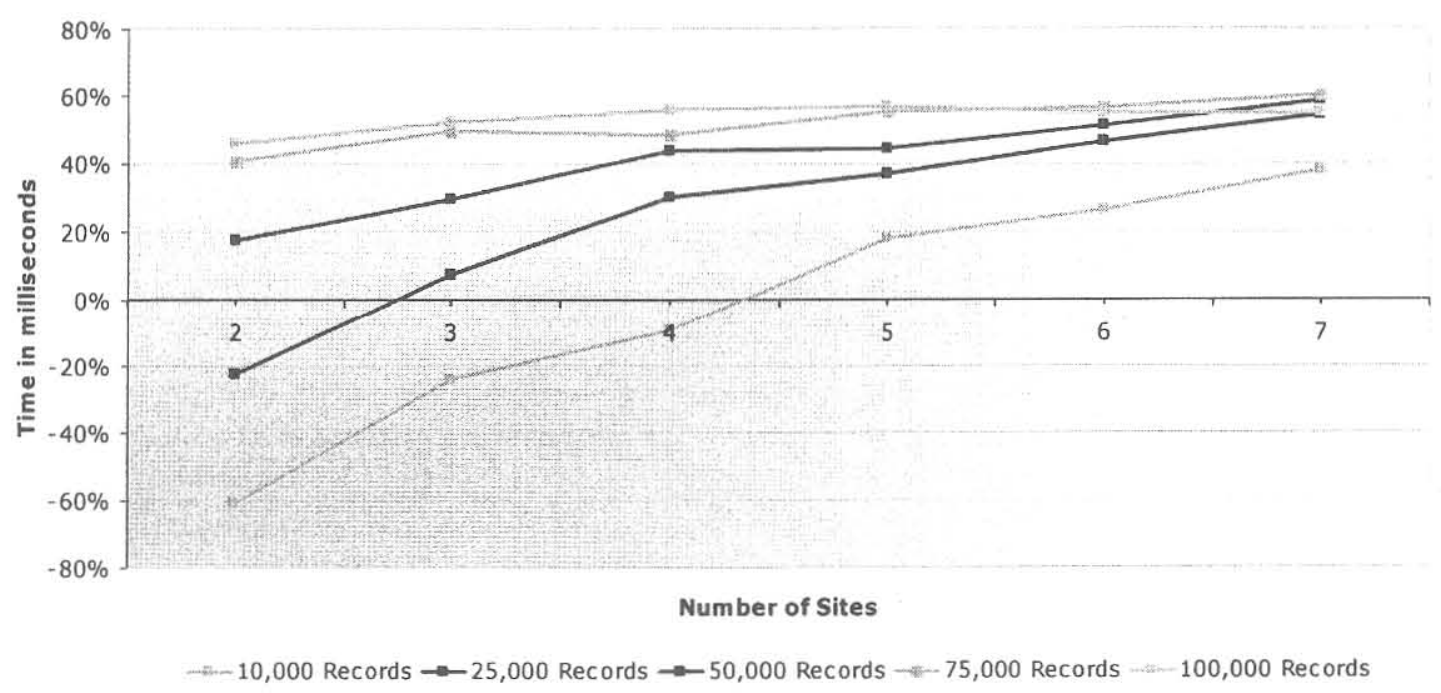

Figure 6.7: Percentage improvement 


\section{CONCLUSION}

This project describes a service oriented distributed data mining framework that provides improved performance and hierarchical structure for developing composite service using core data mining services. This framework neither has any overhead of downloading data to a particular location for mining nor fixed location for data mining tools. In this framework a user will be able to mine data available at multiple sites, using distributed data mining algorithms and executing them on more than one site. The framework allows users ability to discover and use new algorithms and data sets on the Internet and for their data-mining tasks dynamically. The proposed framework alleviates the major limitations of existing approaches:

- The proposed framework allows coordination between different local models. This results in better accuracy in global learning models.

- Aggregate component development allows creation of new data mining components using the existing components. Moreover, the proposed middleware supports integration of heterogeneous technologies, data and algorithms.

- The proposed service oriented approach is dynamic where datasets and algorithms are searched based on the client's requirements. Thus, the location and data format for 
datasets may not be known beforehand. The proposed framework improves the flexibility and performance of a data mining system.

- The proposed service oriented framework is built on open standards and is not tightly coupled but can execute components on heterogeneous platforms and languages. Moreover, Web provides a flexible platform for performing distributed data mining. 


\section{REFERENCES}

[1] ACM SIGKDD, Workshop on Distributed Data Mining, http://www.eecs.wsu.edu / hillol/DKD/dpks2000.html, 2000.

[2] ADELFI: A model for Deployment of High Performance Solutions on Internet/Intranets, Esprit Framework-4 Project, http://ruby.doc.ic.ac.uk/adelfi/, 2000.

[3] Foster I., Kesselman C., Nick J., Tueck S. The Physiology of The Web, http://www.globus.org/research/papers/osga.pdf, 2002.

[4] Bailey S., Grossman R., Sivakumar H., Papyrus: A system for Data Mining over Local and Wide Area Clusters and Super Clusters, http://citeseer. nj.nec.com/408839.html, May 1999.

[5] Ning Chen, Nuno C. Marques, Narasimha Bolloju, AWeb Service-based approach for data mining in distributed environments, CENTRIA, Department of Information FCT, New University of Lisbon, Portugal, http://centria.di.fct.unl.pt/ nchen/papers/iceis.pdf

[6] Moore, Benjamin Seth " Distributed Mathematics as a Web Service," M. Eng. Thesis, University of Louisville, April 2002. (Kumar).

[7] Business Process Execution Language for Web Service, Version 1.1, www.ibm.com/developerworks/libraray/ws-bpel/.

[8] Box, Don, et al. Simple Object Access Protocol (SOAP) 1.1. NC: World Wide Web Consortium, May 2000. http://www.w3.org/ TR/SOAP/, March 2002. 
[9] Chattratichat J., Darlington J., Guo Y., Hedvall S., Köhler M., Syed J., An Architecture for Distributed Enterprise Data Mining, Proceedings of the HPCN Conference, Amsterdam, 1999.

[10] Christensen, Erik, et al. Web Services Description Language (WSDL). NC: World Wide Web Consortium, http://www. w3.org/TR/wsdl.html, March 2001.

[11] Paul Donachy, et al., Web Enabled Distributed Data Mining and Conversion of Unstructured Data, Belfast e-Science Centre, ttp://www.nesc.ac.uk/events/ ahm2003/AHMCD/pdf/070.pdf

[12] Kassie, Fitsum"Smart Card Application Development Using Web Services", M.Engg. Thesis, University of Louisville, Dec. 2003. (Kumar)

[13] Elmaghraby, A. S. , Kantardzic, M and Wachowiak, M. P.," Data Mining From Multimedia Patient Records", book chapter, accepted for publication in the book "Data Mining and Knowledge Discovery Approaches Based on Rule Induction Techniques", editors Triantaphyllou E. and Felici G., Kluwer Academic Publishers, Spring 2004.

[14] Goldszmidt M. and Jensen D., ed., Recommendations Report - DARPA Workshop on Knowledge Discovery, Data Mining and Machine Learning, Carnegie Mellon University, June 1998.

[15] Grossman R., Data Space Project, University of Illinois at Chicago, http://www.dataspaceweb.net/dataspace, January 2000.

[16] Grossman R., S. Bailey, A. Ramu, B. Malhi and A. Turinsky, The Preliminary Design of Papyrus: A System for High Performance, Distributed Data Mining over Clusters, in "Advances in Distributed and Parallel Knowledge Discovery", 
H. Kargupta and P. Chan, editors, AAAI Press/The MIT Press, Menlo Park, California, 2000, pages 259-275.

[17] Grossman, Robert," Integrating Distributed Bioinformatics Data Using Data Webs", Practical Innovation - BioCon 2003, San Diego, CA, February 2003.

[18] Guo Y., Sutiwaraphun J., Integrating Knowledge in Distributed Data Mining, PCW Conference, 1998.

[19] Guo Y., Sutiwaraphun J., Probing Knowledge in Distributed Data Mining, Proceedings of the PAKDD Conference, Beijing, China, 1999.

[20] IEEE IPDSP, Workshop on High Performance Data Mining, http://www.cs.rpi.edu/zaki/HPMD/, 2000.

[21] Kantardzic, M., Data Mining: Methods, Tools and Techniques, IEEE Press and John Wiley, 2002, Pages 380.

[22] Kantardzic M., Kumar A., Toward Autonomic Distributed Data Mining With Intelligent Web Services, Proceedings of The 2003 International Conference on Information and Knowledge Engineering, IKE'03, June 23-26, 2003, Las Vegas, pp. 544-550.

[23] Kantardzic M., Djulbegovic B., Hamdan H., A Data Mining Approach to Improving Polycythemia Vera Diagnostics, Special Issue of Computers and Industrial Engineering Journal on Data Mining and Knowledge Discovery, Vol 43., December 2002, pp. 765-773 .

[24] Kantardzic M., H. Hamdan, B. Djulbegovic, Artificial Neural Networks (ANN) Approach in Diagnostics of Polycythemia Vera, International Journal of Computers and Their Applications, Vol. 8, No. 2, June 2001, pp. 74-79. 
[25] Kantardzic M., Sadeghian P., Knowledge Discovery in Semantically Distributed Data: The Fractal Dimension Approach, submitted for The Nineteenth National Conference on Artificial Intelligence AAAI 2004, San Jose, CA, July 2004.

[26] Kantardzic M., Soliman M., Global Model of Distributed Data Mining is not Summing of Local Models, in "Data Mining $I V$ ", editors: N. Ebecken, C. Breibbia, and A. Zanasi, WIT Press, Southampton, UK, 2004., pp. 89-98.

[27] Kantardzic M.,. Emam A. Z., Elmaghraby A. S., Min H., A Novel Approach for Profile Association Rule Mining from Mixed Databases, The $6^{\text {th }}$ World Multiconference on Systems, Cybernetics, and Informatics SCI 2002, Orlando, Florida, July 2002.

[28] Kantardzic, M., Zurada, J., "New Generation of Data Mining Applications", accepted for publications by IEEE Press and John Wiley, Spring 2004.

[29] Kantardzic, M., Sagedhian, P., Chun, S., "The Time Diversification Monitoring of a Stock Portfolio: An Approach Based on a Fractal Dimension", accepted for the ACM Symposium on Applied Computing SAC 2004, Nicosia, Cyprus, March 2004.

[30] Kantardzic, M., and Badia, A. "Efficient Implementation of Strong Negative Associations Rules", Proceedings of the 2003 International Conference on Machine Learning and Applications, Los Angeles, CA, June 2003, pp. 152-158 (The Best Paper Award).

[31] Kantardzic, M., Badia, A., "A Data Mining Framework for Semantically Distributed Databases", Proceedings of The Seventh IASTED International Conference in Artificial Intelligence and Soft Computing (ASC 2003), Banff, Canada, July 2003. 
[32] Kubera, F. etal, "Unraveling the Web Service Web: An introduction to SOAP, WSDL, UDDI", IEEE Internet Computing, Vol. 6, No. 2, March/April 2002.

[33] Kantardzic, M., Soliman, M., "An Approach for Mining Frequent Sequences in Distributed Environment", Proceedings of the 2002 International Conference on Information and Knowledge Engineering, Las Vegas, Nevada, June 2002.

[34] Kargupta H., "Distributed Knowledge Discovery from Heterogeneous Sites", DIADIC Laboratory, University of Maryland at Baltimore County, http://www.cs.umbc.edu/ hillol.

[35] Kargupta H., Chan P., Advances in Distributed and Parallel Knowledge Discovery, AAAI Press/ MIT Press, 1999.

[36] Kargupta H., Park B., Johnson E., Hershberger D., Huang W., Ayyagari R., Ghosh S., Distributed Knowledge Discovery from Heterogeneous Sites, Project Proposal, http://www.cs.umbc.edu/ hillol/DKD/ddm_research.html.

[37] Kargupta H., Park B., Hershberger D., Johnson E., Collective Data Mining: A New Perspective Toward Distributed Data Mining, in "Advances in Distributed and Parallel Knowledge Discovery", eds.: Hillol Kargupta and Philip Chan, MIT/AAAI Press, 1999.

[38] Krishnaswamy, S., Zaslasvky, A., and Loke, S, W., Internet Delivery of Distributed Data Mining Services: Architectures, Issues and Prospects, Chapter 7 in the book Architectural Issues of Web-enabled Electronic Business, Murthy, V.K. and Shi, N. (eds.), 2003, pp. 113 - 127, Idea Group Publishing.

[39] Krishnaswamy, S., Loke, S, W., and Zaslavsky, A., Towards Anytime Anywhere Data Mining E-Services, Proceedings of the Australian Data Mining Workshop 
$\left(A D M^{\prime} 02\right)$ at the 15 th Australian Joint Conference on Artificial Intelligence, (eds)

S.J. Simoff, G.J. Williams, and M. Hegland. Canberra, Australia, December 2002, pp. $47-56$.

[40] Kumar, A., Kantardzic, M., Ramaswamy, P., Sadeghian, P., "An Extensible Service Oriented Distributed Data Mining Framework", Proceedings of International Conference on Machine Learning and Applications ICMLA04, Louisville, December 2004, pp. 161-169.

[41] Little, M., "Transactions and Web Services", Communications of the ACM, Vol. 46, No. 10, pp.29-34, 2003.

[42] Kumar, A., Kantardzic, M., "Web Application Protocols and Services for Distributed Data Mining", The Ninth ACM SIGKDD International Conference on Knowledge Discovery and Data Mining - Workshop on Data Mining Standards, Services and Platforms (DM-SSP 03), Washington, DC, August 2003.

[43] McKarthy J., Phenomenal Data Mining: From Data to Phenomena, SIGKDD Explorations, Vol. 1, No. 2, 2000, pp. 24-29.

[44] Papazoglou, M.P. and Georgakopoulos, D. ," Service Oriented Computing," Communications of the ACM, Vol. 46, No. 10, pp. 25-28, 2003.

[45] Park , B. H., Kargupta H, "Distributed Data Mining: Algoriths, Systems and Applications", http://citeseer.nj.nec.com/540909.html, 2000.

[46] Parsarthy, S. and Subramanian, R. "An Interactive Resource aware Framework for Distributed Daya mining", Newsletter of the IEEE Technical Committee on Distributed Processing, Spring 2001, pp. 24-32. 
[47] Prodromidis A. L., Chan P. K., Stolfo S. J., "Meta-Learning in Distributed Data Mining Systems: Issues and Approaches", In "Advances in Distributed and Parallel Knowledge Discovery ", Kargupta and Chan (eds.), MIT/AAAI Press, 1999.

[48] Software Architecture for DecisionCentre: A Web-based Distributed Data Mining System, Data Mining Group, Imperial College, PCW, 1997.

[49] Sayal, Mehmet and Scheuermann, Peter, “ A Distributed Clustering Algorithm for Web-Based Access Patterns", in Proceedings of the 2nd ACM-SIGMOD Workshop on Distributed and Parallel Knowledge Discovery, Boston, August 2000.

[50] Kantardzic, M., et al., "Data Mining Approach in a Selection of Laparoscopic Techniques", Proceedings of the $10^{\text {th }}$ International Conference on Intelligent Systems, Arlington, VA, June 2001, pp. 1-4.

[51] Soliman, M., Kantardzic, M., "Towards Building a Distributed Mining Approach", Proceedings of the 2nd IEEE International Symposium on Signal Processing and Information Technology, Marrakesh, Marocco, December 2002, pp. 305-309.

[52] Steve J. , "A Wireless Printing Application Using Web Service Technology", M.S. Thesis, University of Louisville, May 2002, (Kumar).

[53] Subramonian R., Parthasarathy, "An Architecture for Distributed Data Mining", Fourth International Conference on Knowledge Discovery and Data Mining, New York, 1998, pp. 44-59. 


\section{APPENDIX \\ Publish.java - This program lets the algorithm/data service providers to publish their service with the registry.}

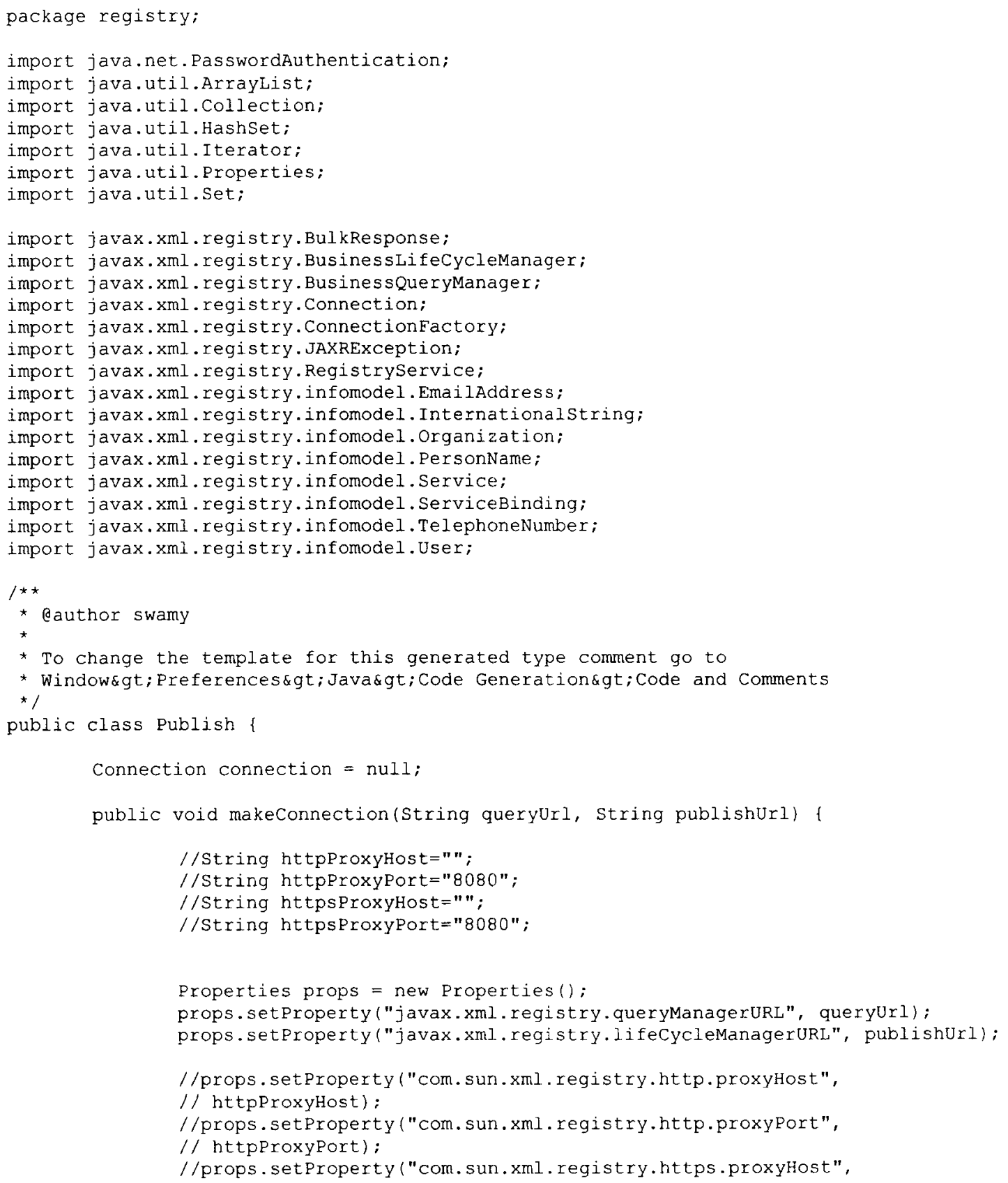




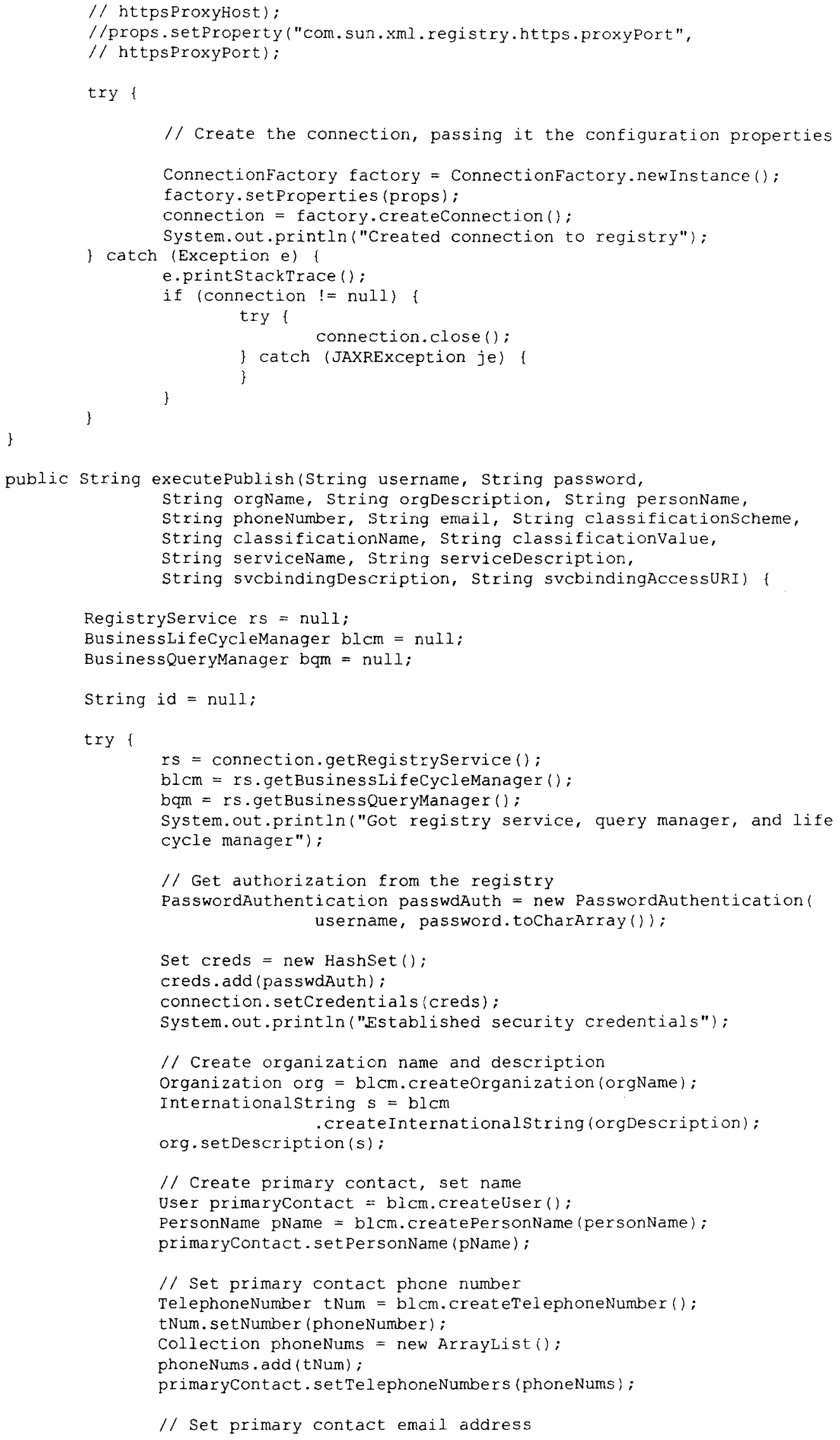




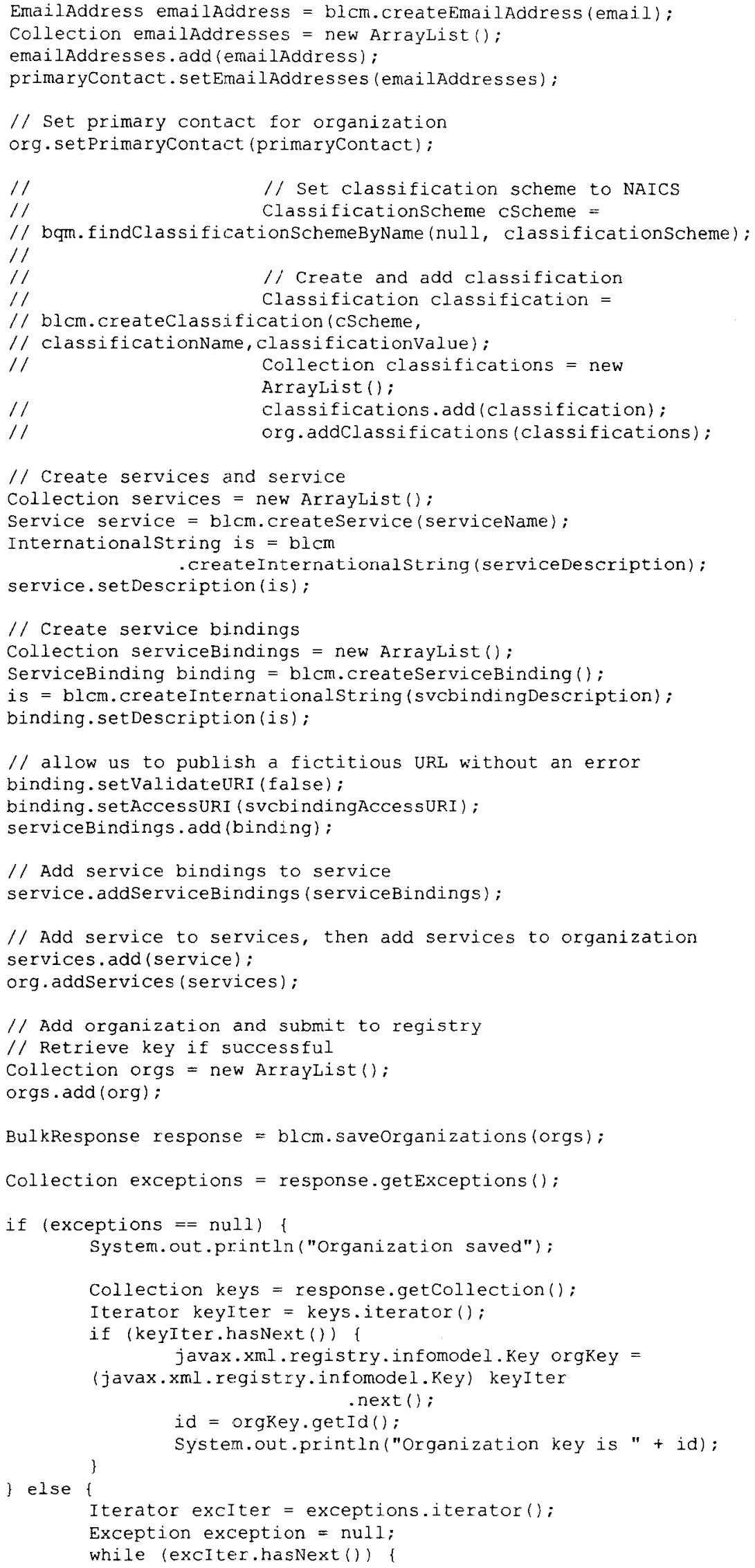




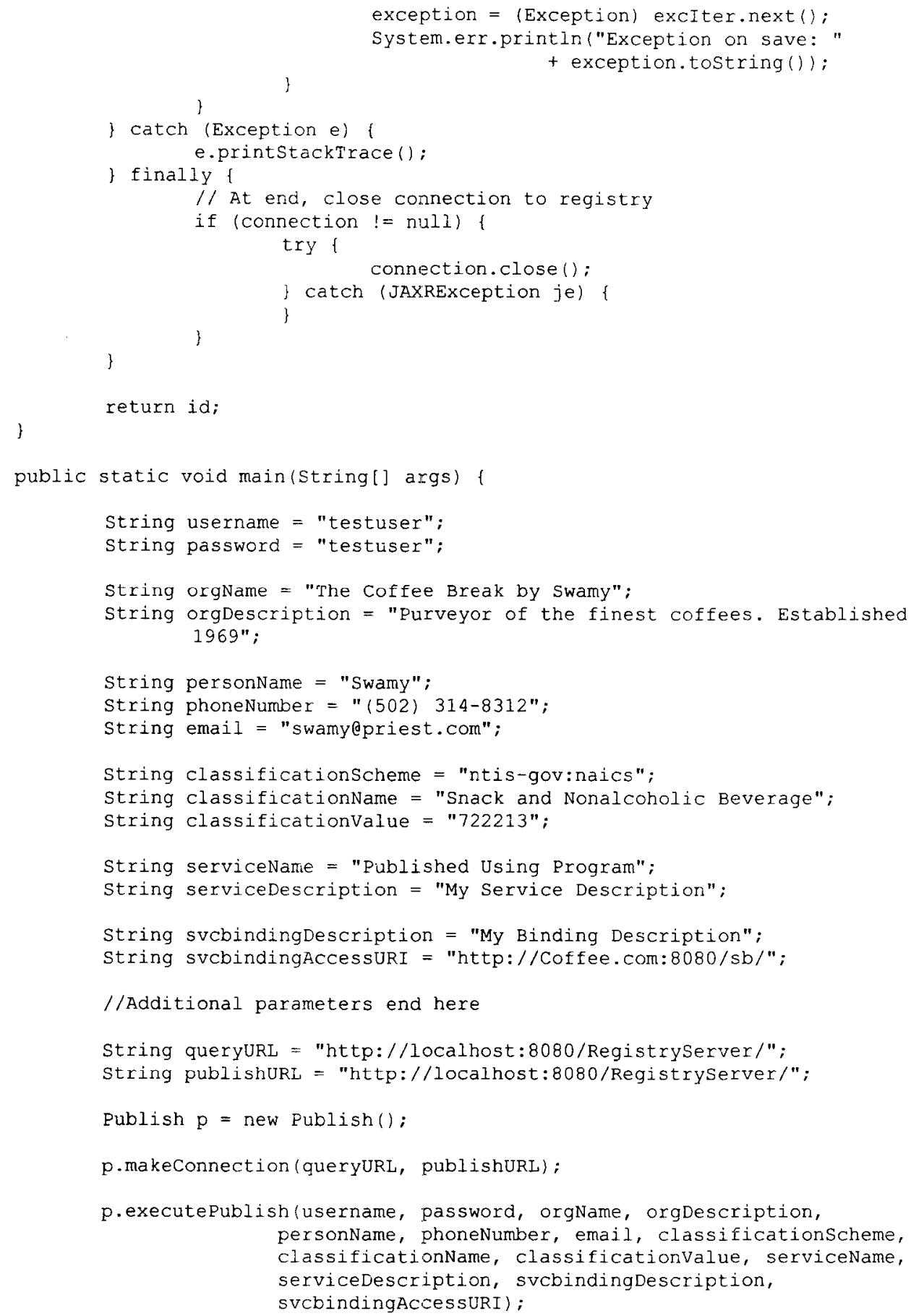


QueryRegistry.java - This program lets the algorithm/data service providers to publish their service with the registry.

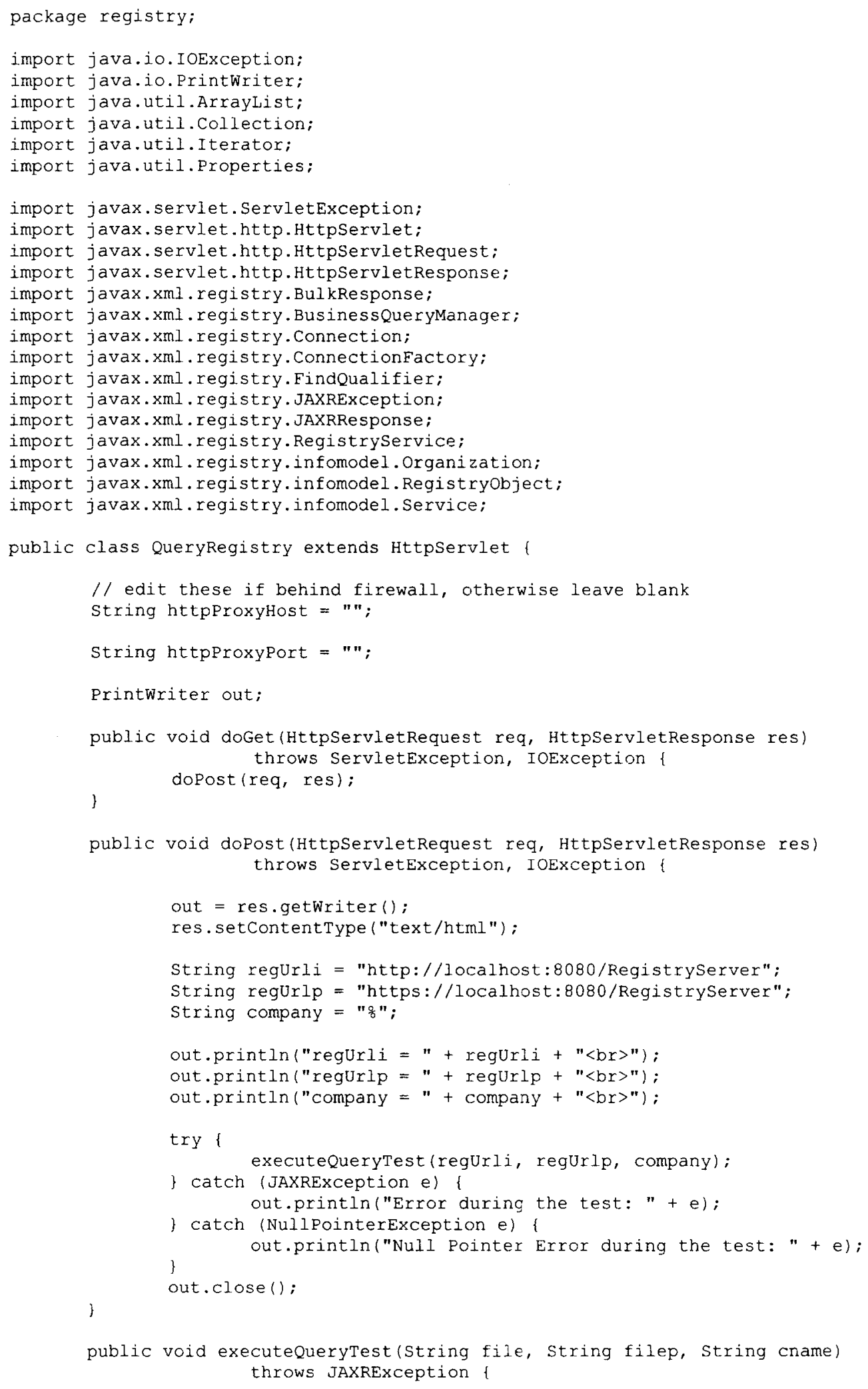


Properties props = new Properties();

props.setProperty ("javax.xml.registry.queryManagerURL", file);

props.setProperty ("javax.xml.registry.lifecycleManageruRL", filep); props.setProperty ("javax.xml.registry.factoryclass", "com.sun.xml registry.uddi. ConnectionFactory Impl");

props.setProperty ("com.sun.xml .registry.http.proxyHost", httpProxyHost);

props.setproperty ("com.sun.xml.registry.http.proxyport", httpproxyPort);

Connectionfactory factory = Connectionfactory.newInstance(); factory. setproperties (props);

Connection conn = factory.createconnection();

RegistryService rs = conn.getRegistxyService();

BusinessQueryManager bqm = rs.getBusinessQueryManager();

Arraylist names $=$ new Arraylist ();

names.add (cname);

Collection fQualifiers = new ArrayList();

fQualifiers, add(FindQualifier.SORT BY NAME DESC);

BulkResponse br = bqm.findorganizations(fQualifiers, names, null, null, null, null):

if (br.getStatus() $==$ JAXRResponse.STATUS_SUCCESS) 1 out.println("Successfully queried the "

+ "registry for organization matching the "

"<br>");

+ "name pattern: \" + cname + "\" +

Collection orgs = br.getcollection( $)$

out.println("Results found: " + orgs.size() + "<br $>$ ");

Iterator iter = orgs.iterator();

while (iter.hasNext()) (

Organization org = (Organization) iter.next (); "<br>"): out.printIn("Organization Name: " + getName(org) + org.getkey() getId() out.printin("Organization Key: "+

$$
+"<\mathrm{b} r>") ;
$$

out.println("Organization Description:"

+ getDescription(org) $+"<$ br $>")$;

Collection services = org.getservices();

Iterator siter = services.iterator();

while (siter.hasNext()) (

Service service = (Service) siter next) ): out.println("\tService Name: "+ getName (service) $\quad+"<\mathrm{br}>"$ ) out.println("\tService key: "

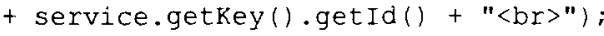
out.println("\tService Description: " + getDescription (service) + "<br>");

\} else \{ out.println("One or more JAXRExceptions " + "occurred during the query operation:"); Collection exceptions = br.getExceptions (); Iterator iter = exceptions.iterator(); while (iter.hasNext()) (

Exception e = (Exception) iter.next);

\} out.println(e.tostring());

catch (JAXRException e)

e.printstackTrace (); 


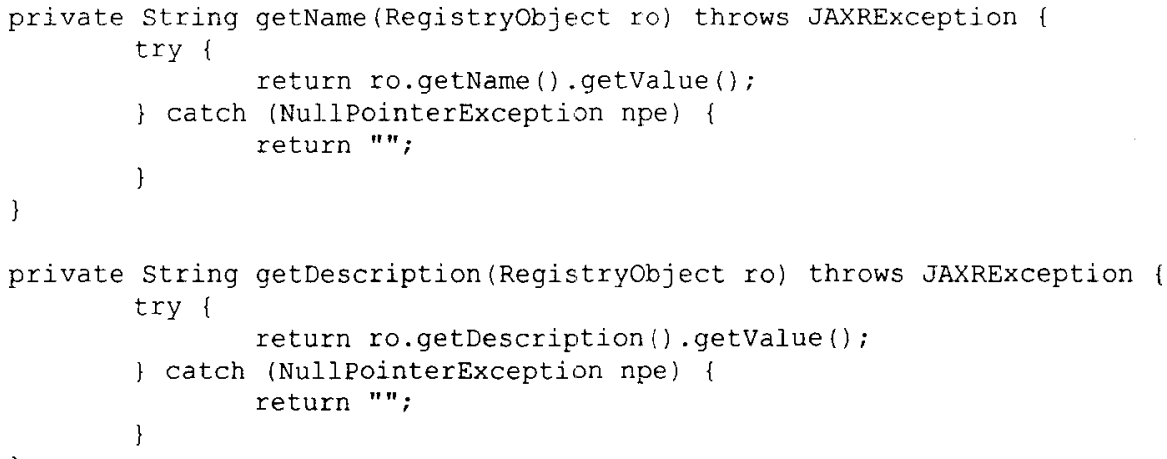

ShowDataSets.java - This program displays the data sets published in the registry based on the search performed by the user.

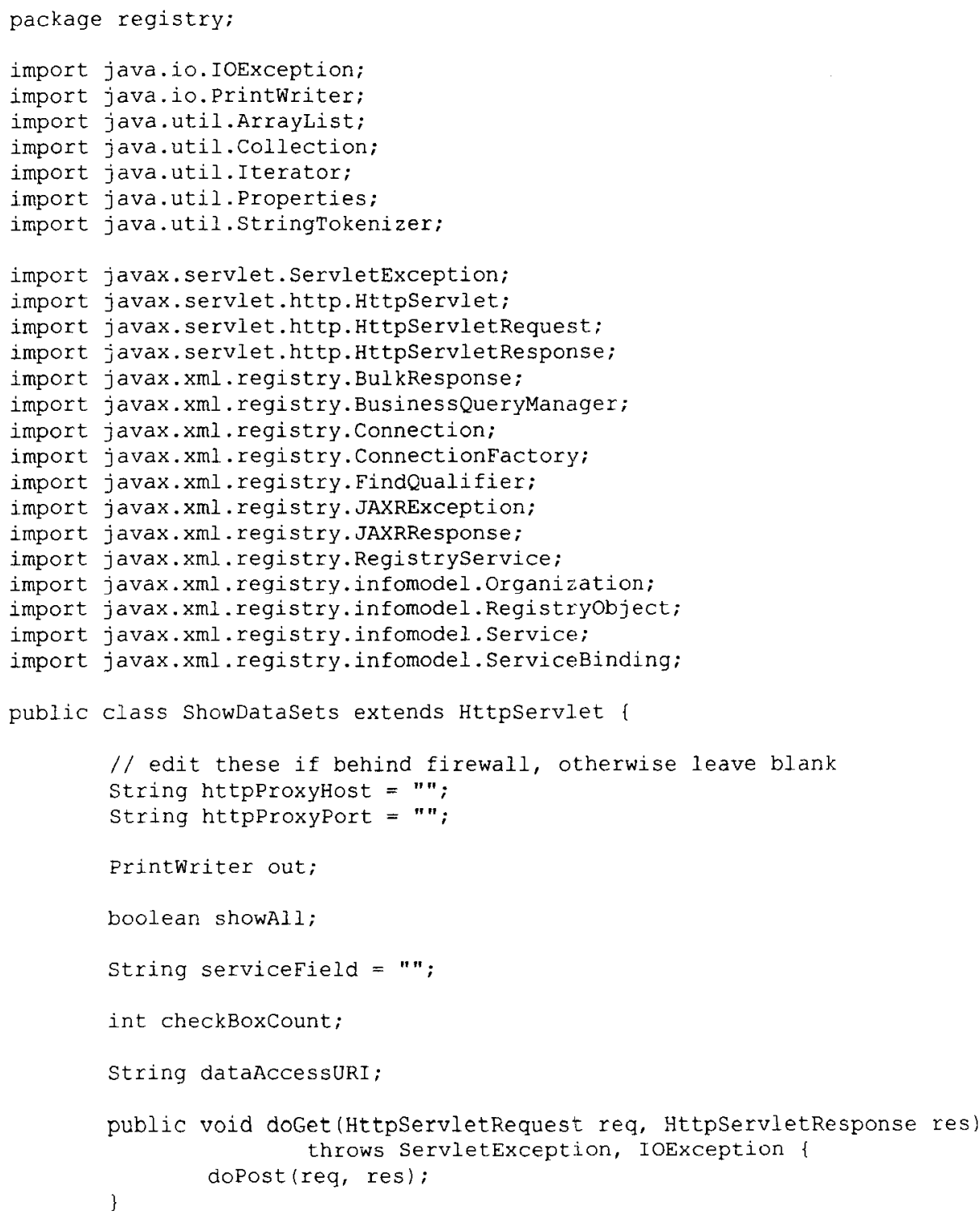


public void dopost(HttpservletRequest req, HttpservletResponse res) throws ServletException, IOException

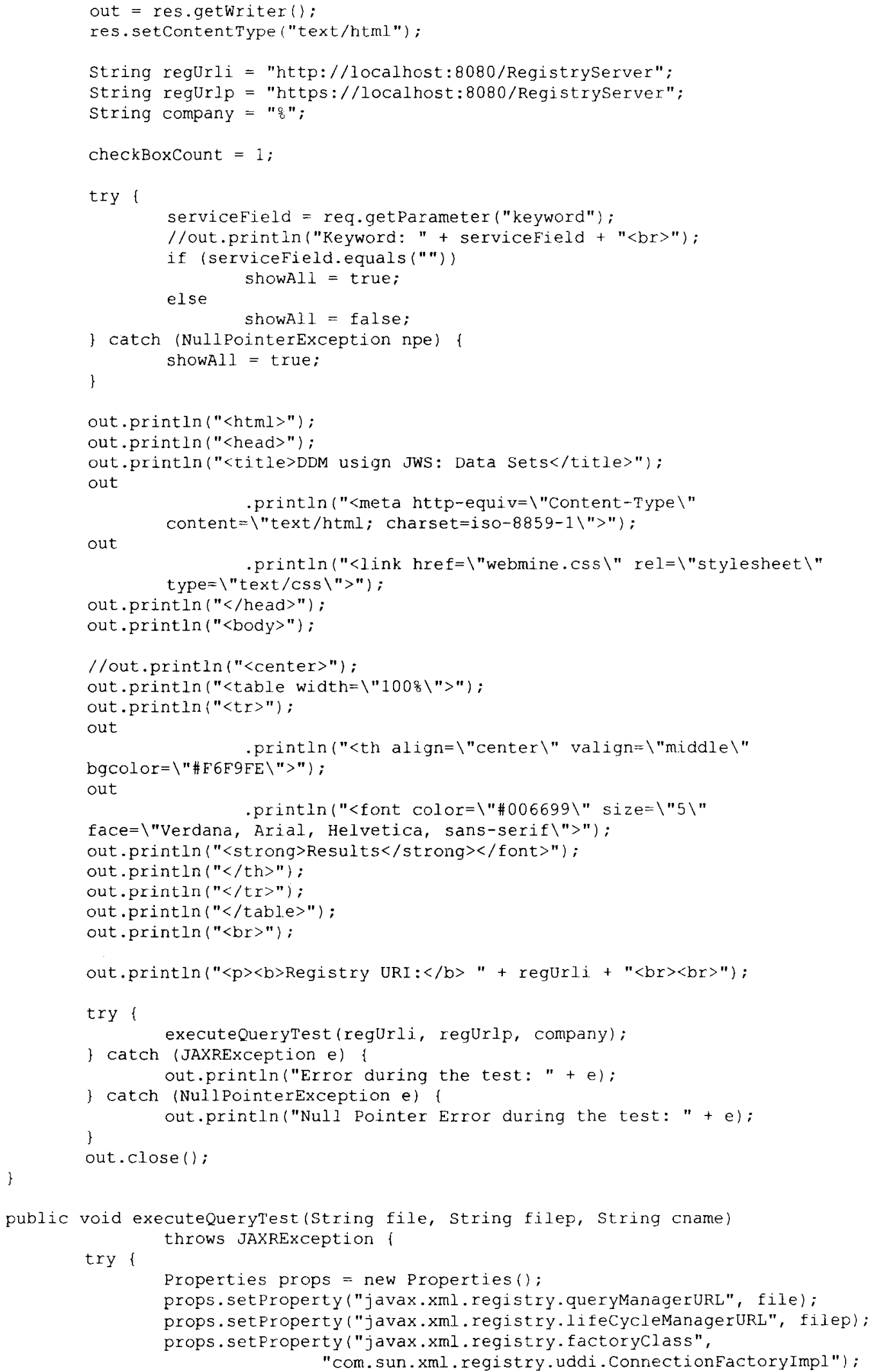




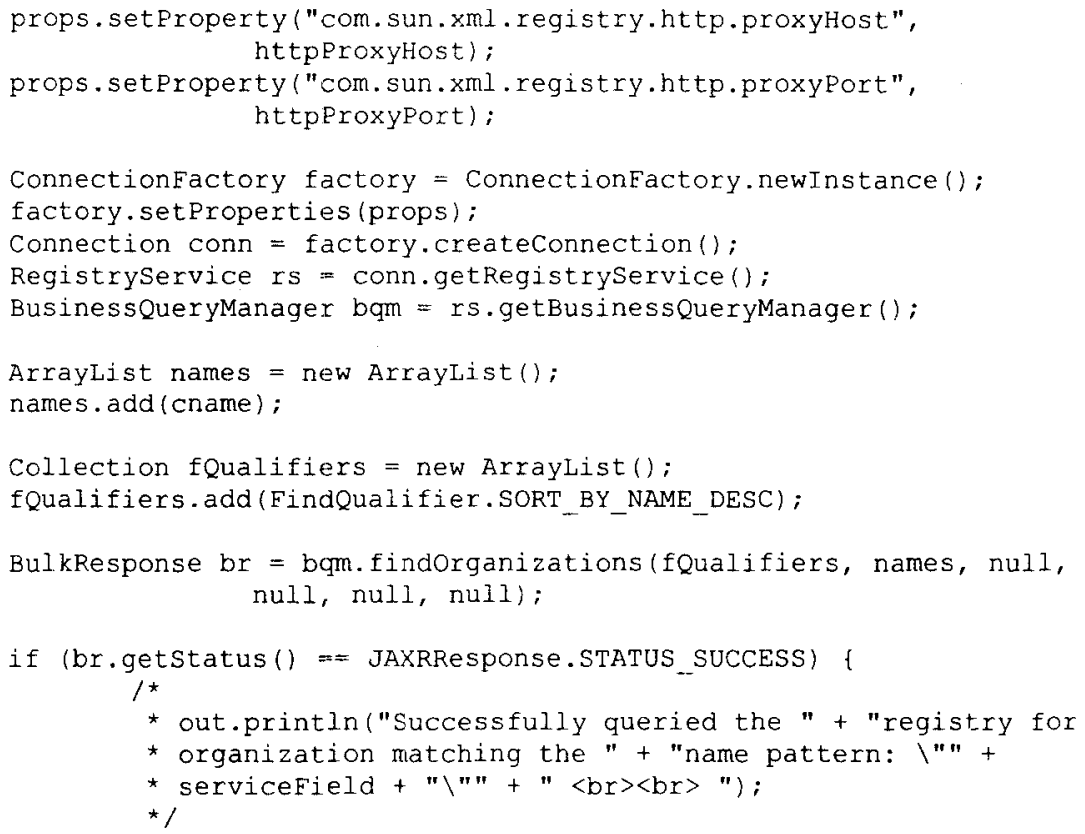




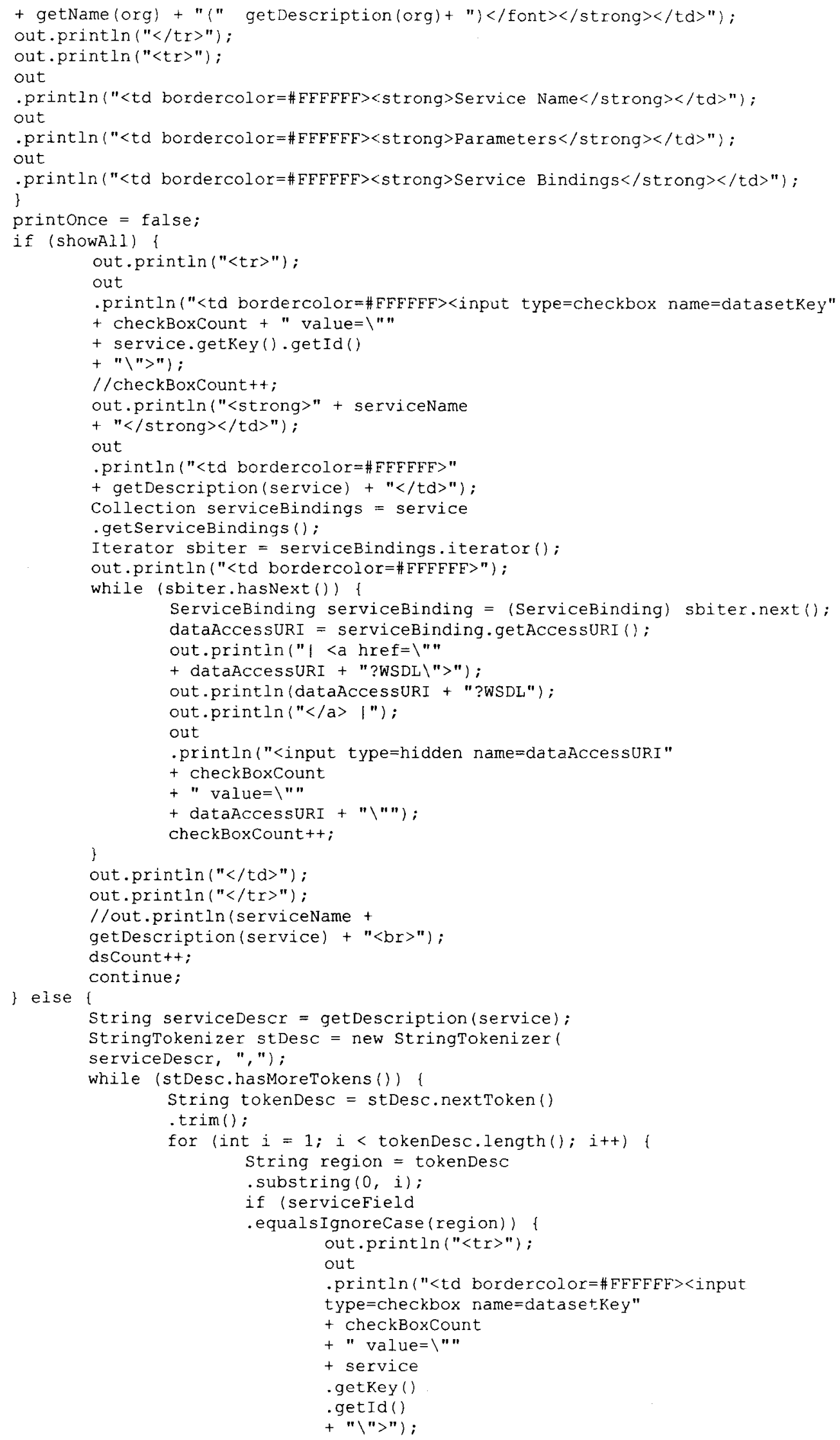




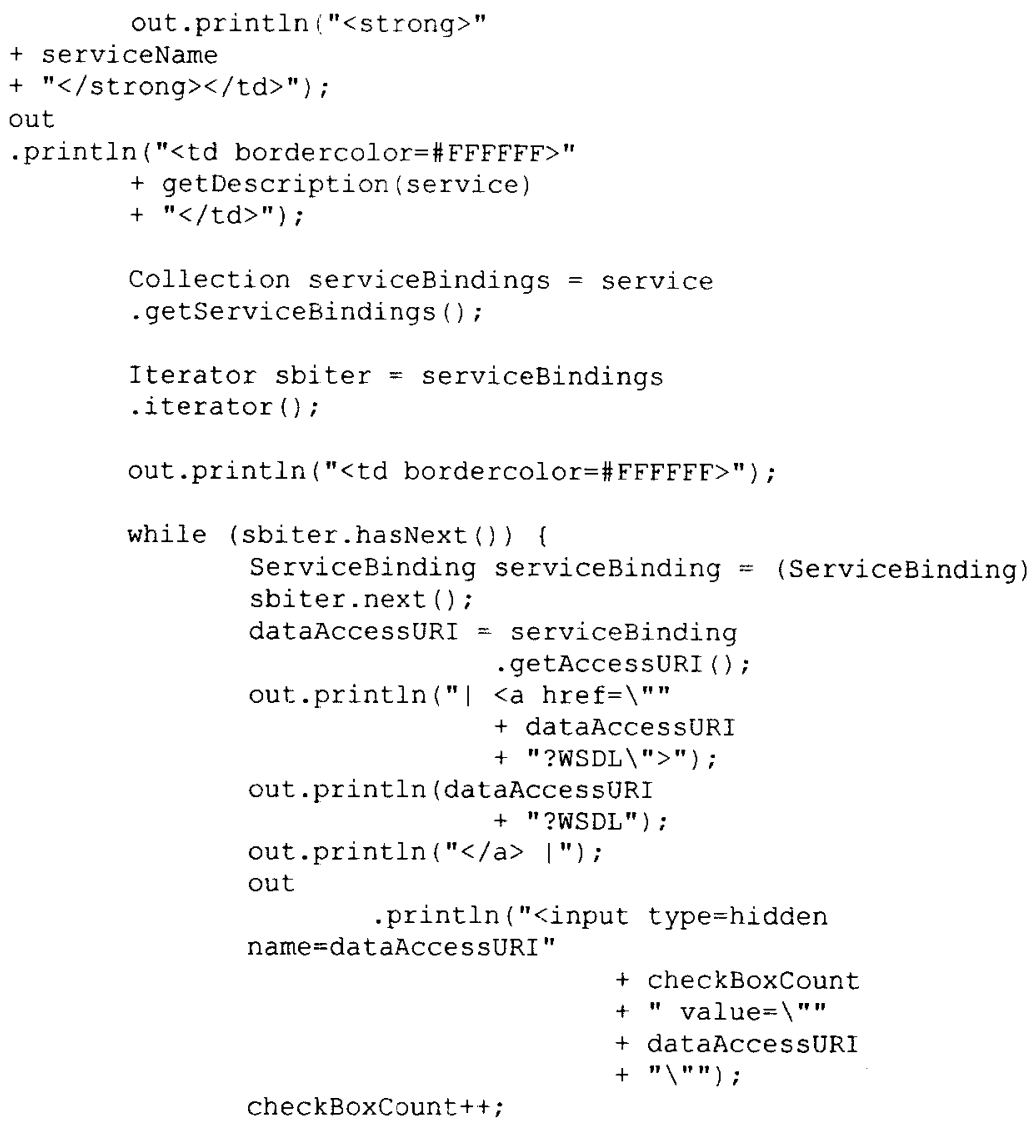

\}

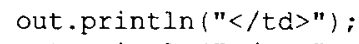




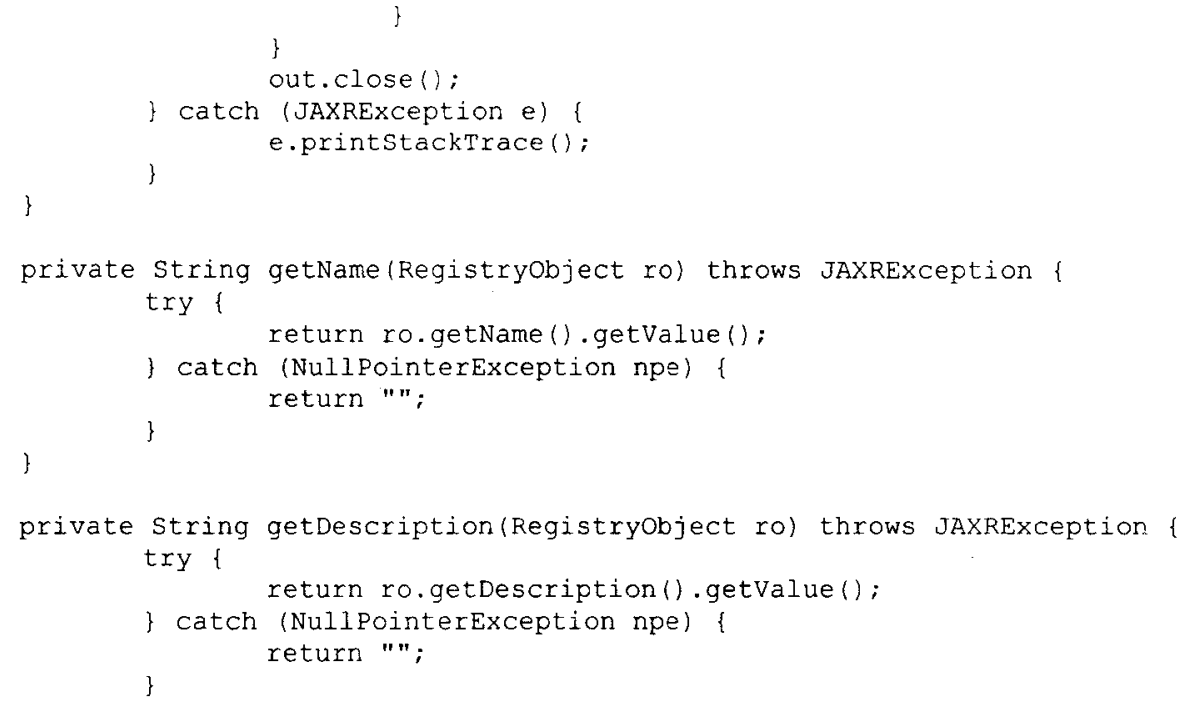

ShowAlgorithms.java - This program queries the registry and displays the appropriate algorithms based on the data sets selected by the user.

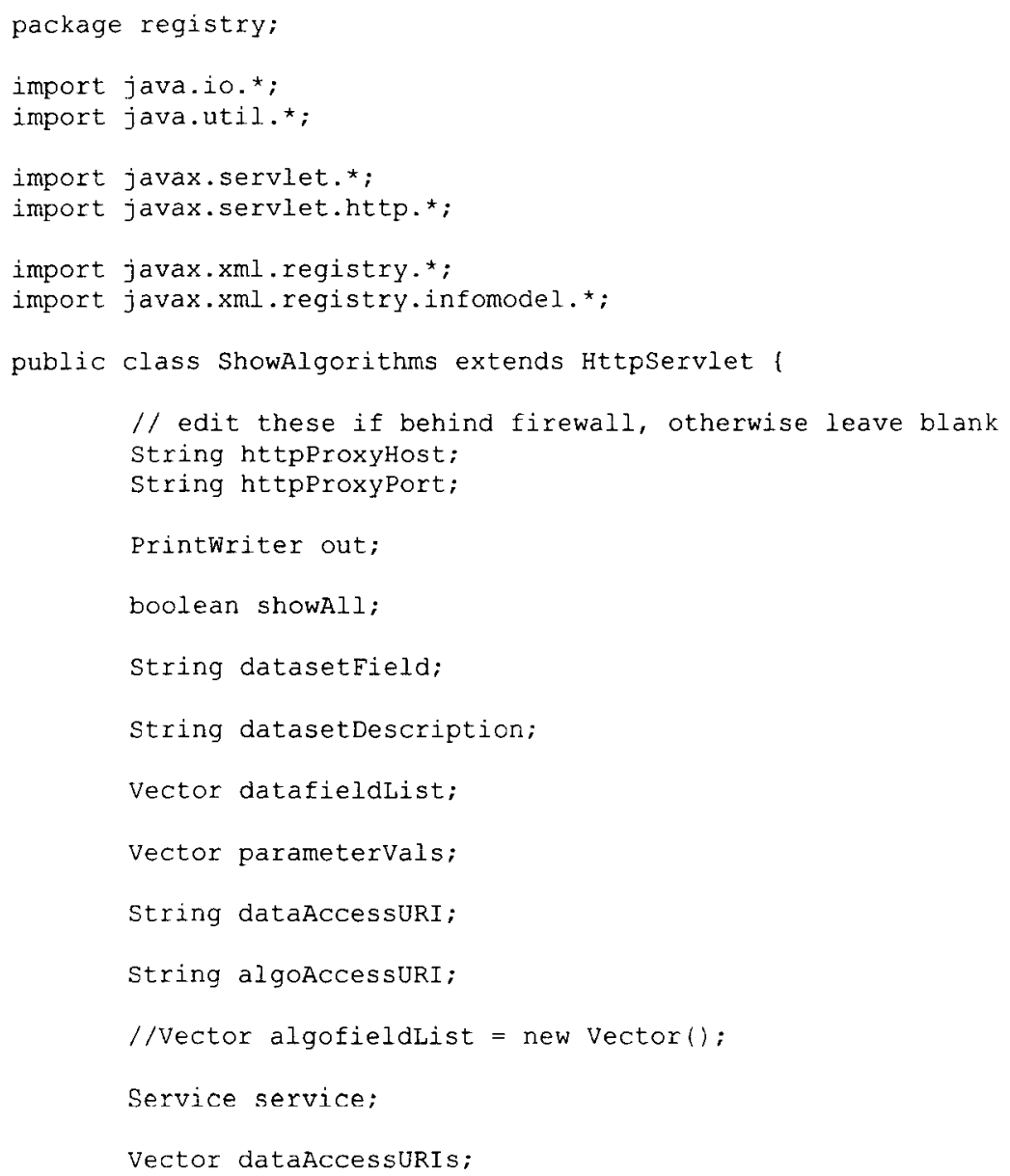




\section{Vector algoAccessURIs;}

public void doGet (HttpservletRequest req, HttpservletResponse res) throws ServletException, IOException i

) dopost(reg, res);

public void doRost(HttpservletRequest req, HttpservletResponse res) throws ServletException, IOException 1

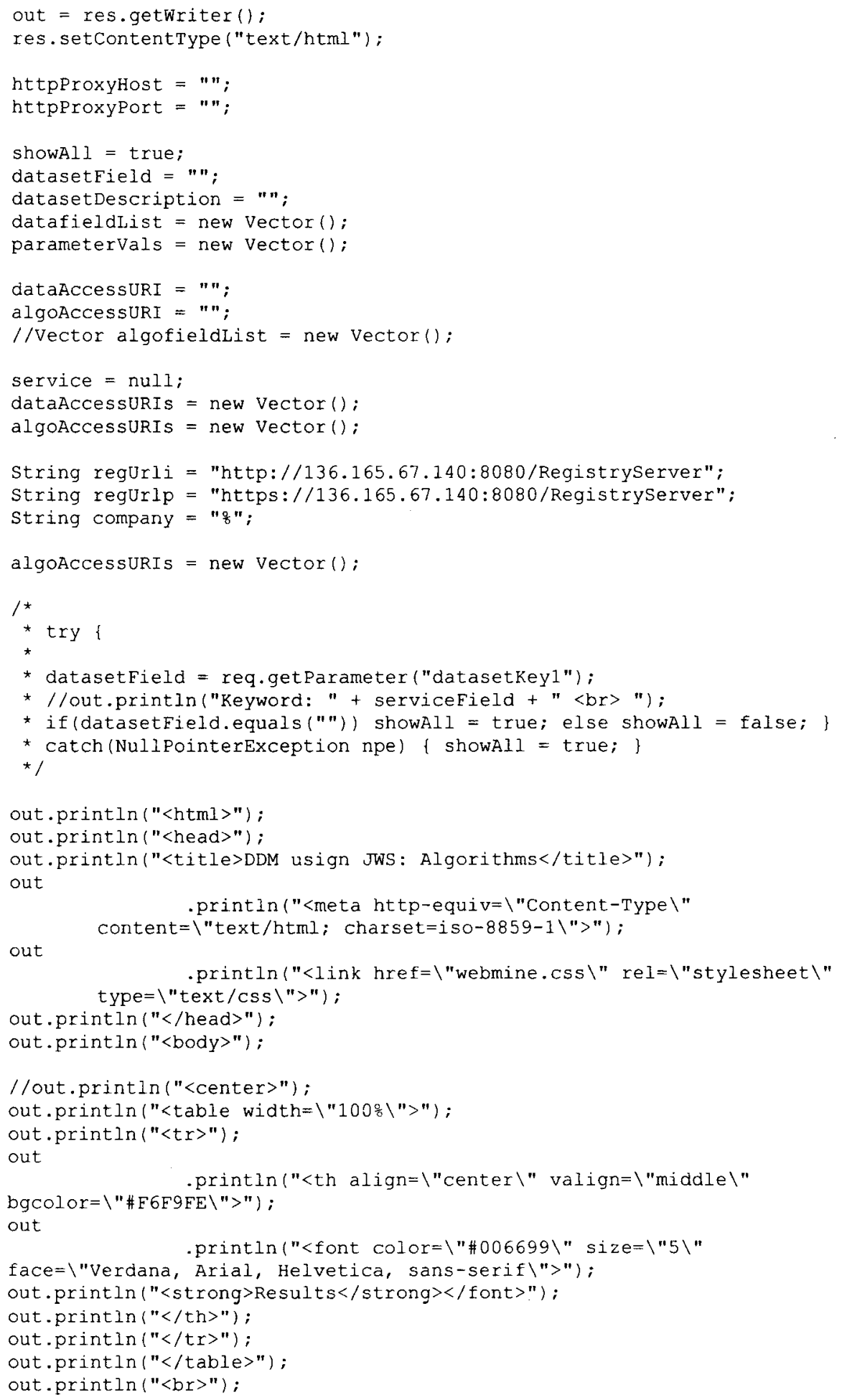




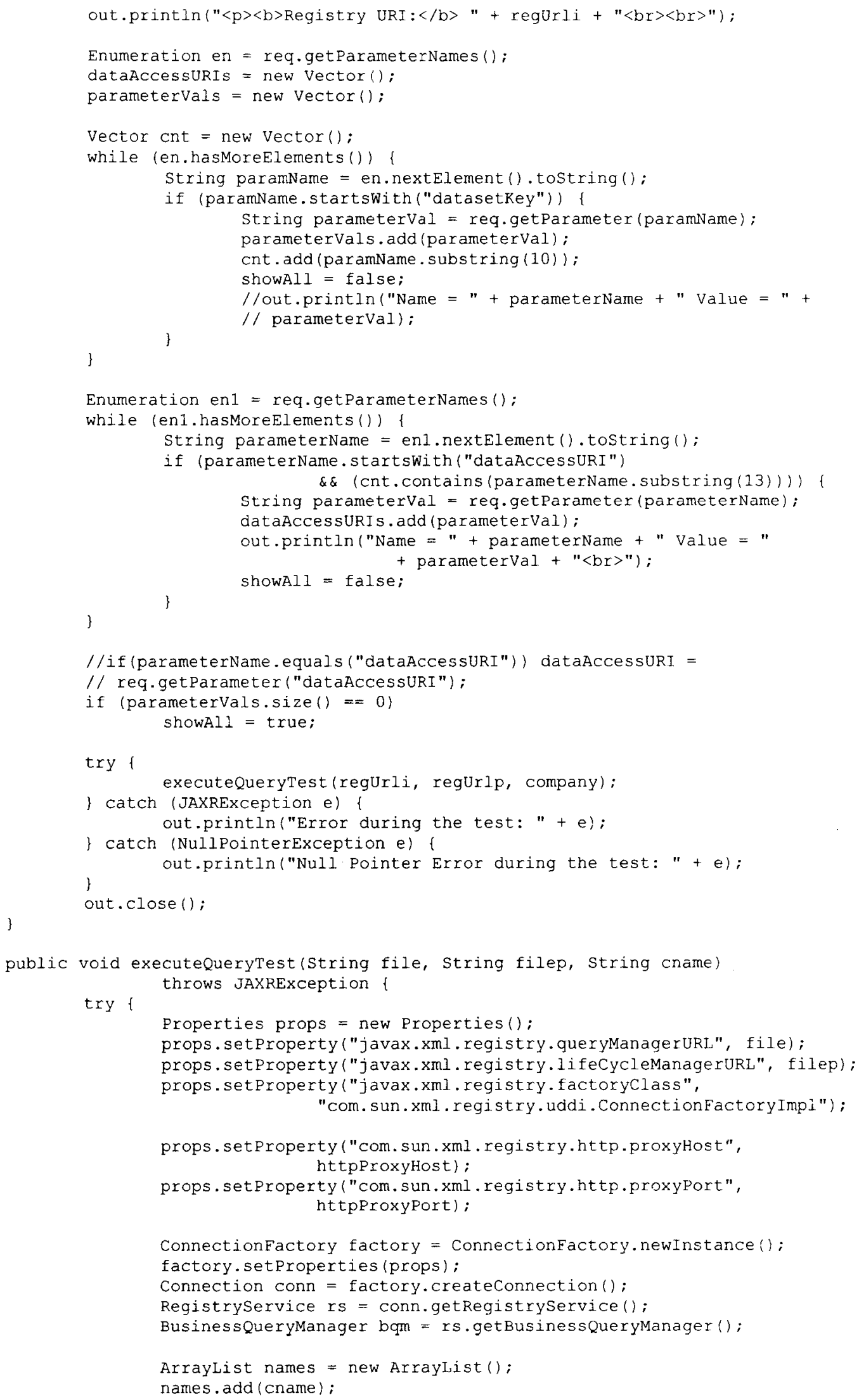


datafieldList $=$ new vector ():

Collection EQualifiers = new ArrayList();

fQualifiers.add (FindQualifier.SORT_BY_NAME_DESC);

BulkResponse br = bqm. findorganizations (fQualifiers, names, null, null, null, null);

if (br.getstatus () $==$ JAXRResponse.STATUS_SUCCESS)

out

-println ("<form name=algorithmform

method=post action=webMineServlet $>$ ");

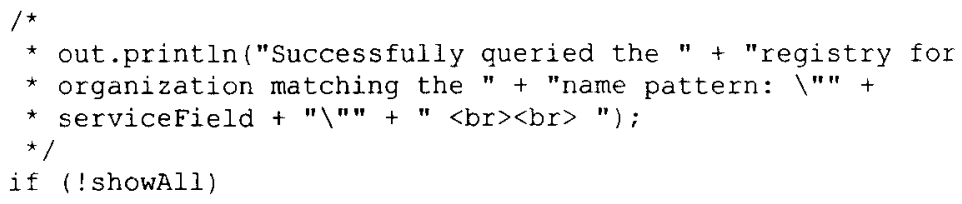


out.printIn("<td bordercolor=\#FFFFFF><strong $>$ Parameters: $\langle/$ strong $\rangle\langle$ br $\rangle ")$;

StringTokenizer serviceparameters = new stringTokenizer

getDescription (tempservice), ",");

while (serviceparameters.hasmoreTokens ()) \{

String attribute = serviceparameters

nextToken ();

out.println("<input type=radio name=l"attribute--"

+ dataAccessuRIs.get (i)

+ "\" value="

+ attribute

$+">")$;

out.println(attribute $+"\langle b r\rangle ")$;

\}

out.println ("</td $>$ ");

out.printin("</tr>");

out.println("</table $>$ ");

out.printin ("<br>");

StringTokenizer datasetTokens = new StringTokenizer(

datasetDescription);

while (datasetTokens.hasMoreTokens()) (

String datasetToken = datasetTokens

- nextToken ();

. indexof(": ");

int indexofColon = datasetToken

/ /out.printin(indexofcolon + "<br $>$ ")

string fieldType = datasetToken.substring

indexOfColon +1 , datasetToken

- length());

if (fieldType.endswith(","))

fieldType = fieldType.substring $(0$,

fieldType.length() - 1):

/ lout.println (fieldType + "<br>");

datafieldList.add (fieldType.trim()

. tolowercase ()) ;

)

/ /out.printin("<strong>Data Field

$/ /$ List:</strong $>$ + datafieldList +

/ " $<$ br $><$ br $>$ ") ;

out

.println ("<font face=verdana size=4px $\rangle\langle$ strong $\rangle$ Algorithms $\langle/$ strong $\rangle\langle/$ font $\rangle "$ ) ;

collection orgs = br.getcollection( );

Iterator iter = orgs.iterator ();

int dscount $=0$;

for (int $i=0 ; i<$ parametervals.size(); $i++$ )

out.println ("<input type=hidden name=datasetkey" + i

+ "value=" + parametervals.get $(i)+">$ ");

out.print In ("<table border $=1>$ ");

while (iter.hasNext()) (

boolean printonce = true;

Organization org $=$ (Organization) iter.next();

Collection services = org.getServices();

Iterator siter = services.iterator ();

while (siter.hasNext()) (

service $=$ (Service) siter.next ();

String servicename = get $\mathrm{Name}$ (service);

stringtokenizer st $=$ new

StringTokenizer (servicename);

while (st.hasmoreTokens ()) (

String token $=$ st. nextToken ()$;$ if

(token.equals Ignorecase ("Algorithm")

1

token.equalsIgnoreCase ("Algorithms")) (

if (printonce) ? 


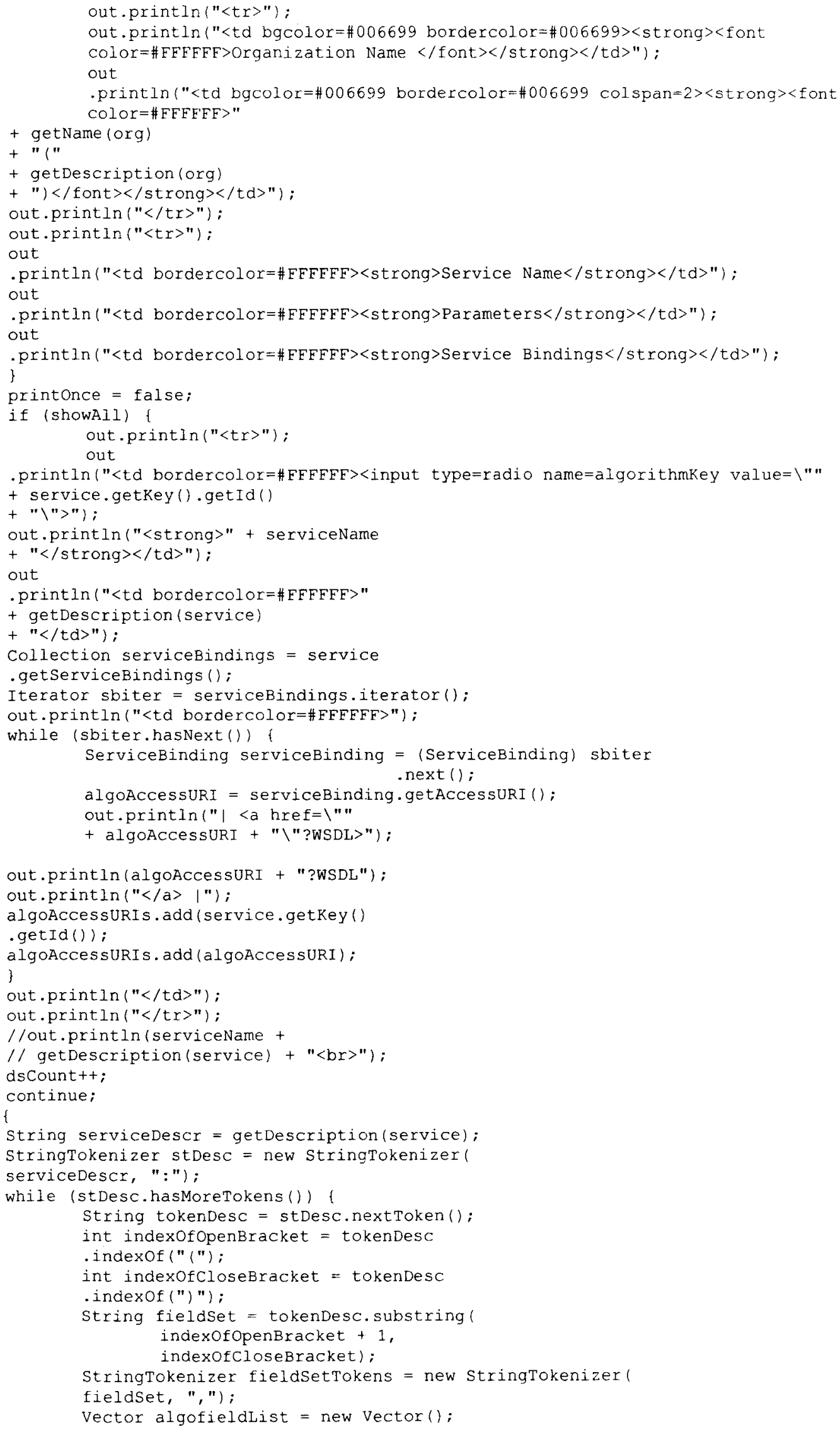


while (fieldSetTokens.hasMoreElements()) (

algofieldList.add(fieldsetTokens

-nextToken().trim()

.tolowerCase());

子

Collections. sort (algofieldList);

Collections. sort (datafieldList);

//out.println("Algo Field List: "+

$/ /$ algofieldList + "<br $\rangle ")$;

//out.println("Data Field List: "+

// datafieldList + "<br $>$ ");

if (new Subset(). compare(datafieldList,

algofieldList) \{

out.println ("<tr>");

out

.println ("<td bordercolor=\#FFFFFF $><$ input type=radio name=algorithmkey value $=\backslash$ "

+ service.getkey()

-getia()

$+"(">")$;

out.println("<strong>"

+ servicename

+ " $<$ (strong $></$ td $>")$;

out

.println ("<td bordercolor=\#FFFFFF>"

+ getDescription (service)

+ " $</$ td $>")$;

Coliection serviceBindings = service

.getServiceBindings();

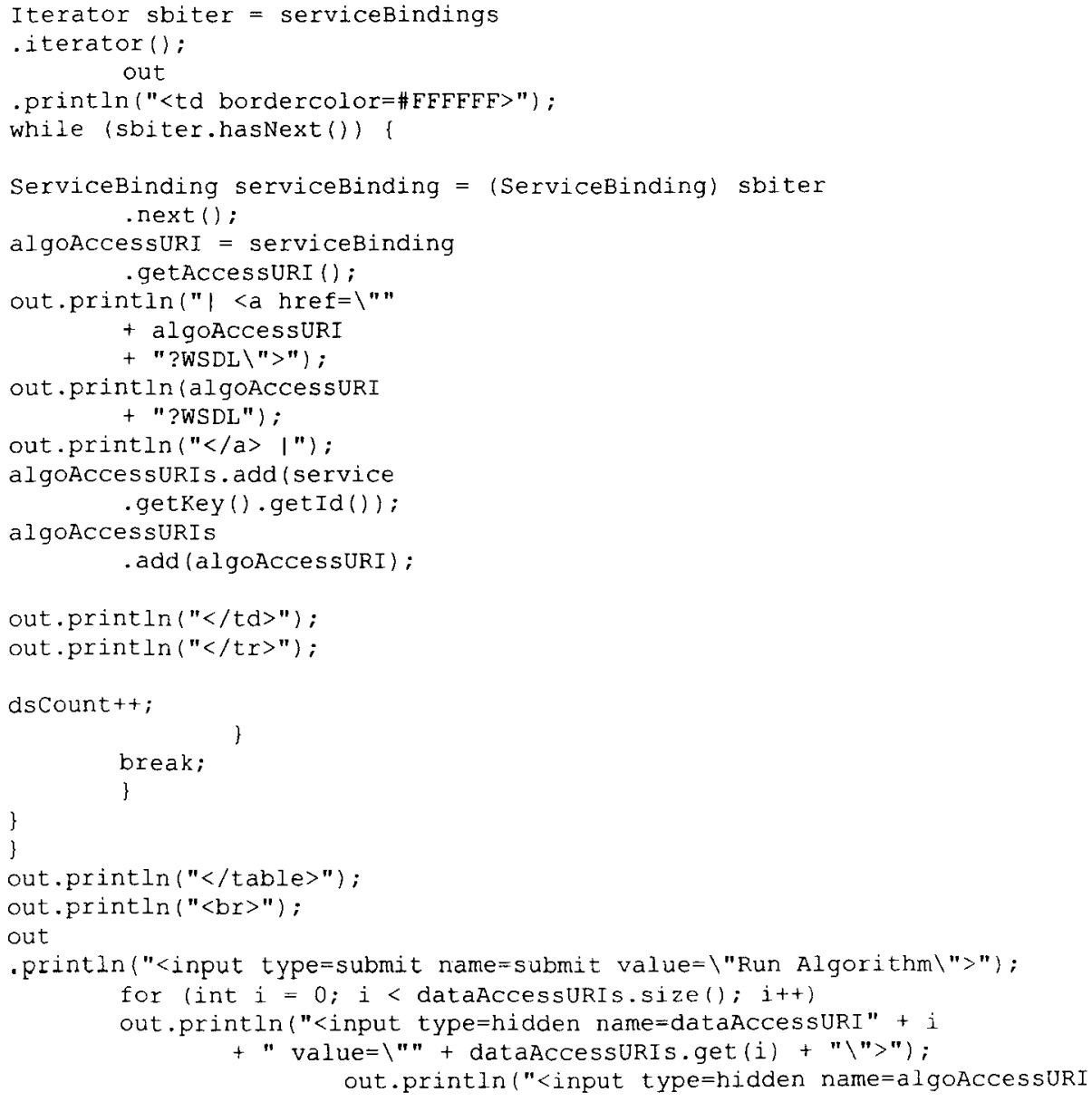




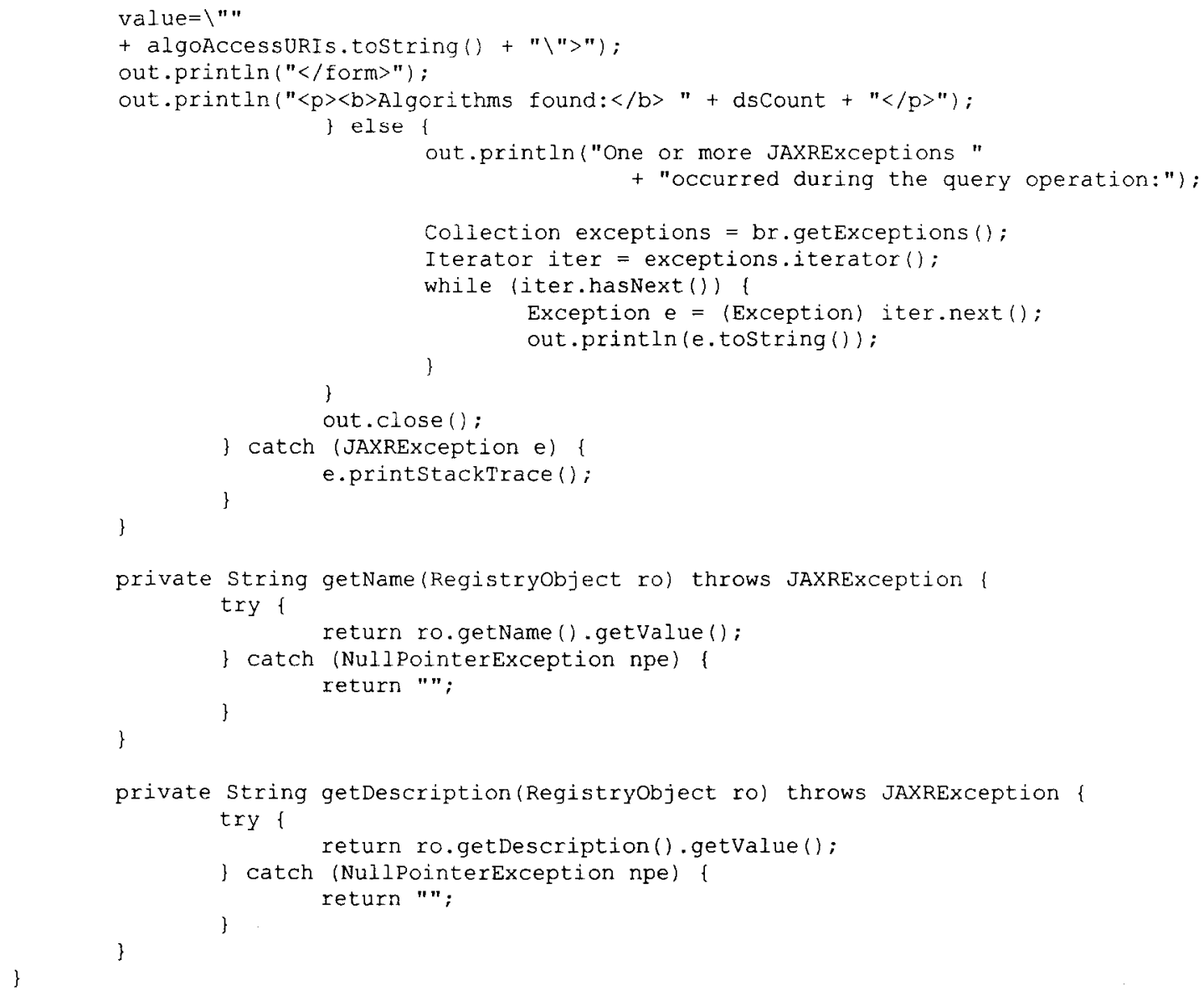

\section{Apriorilmpl.java - Implementation of the Apriori algorithm as a web service.}

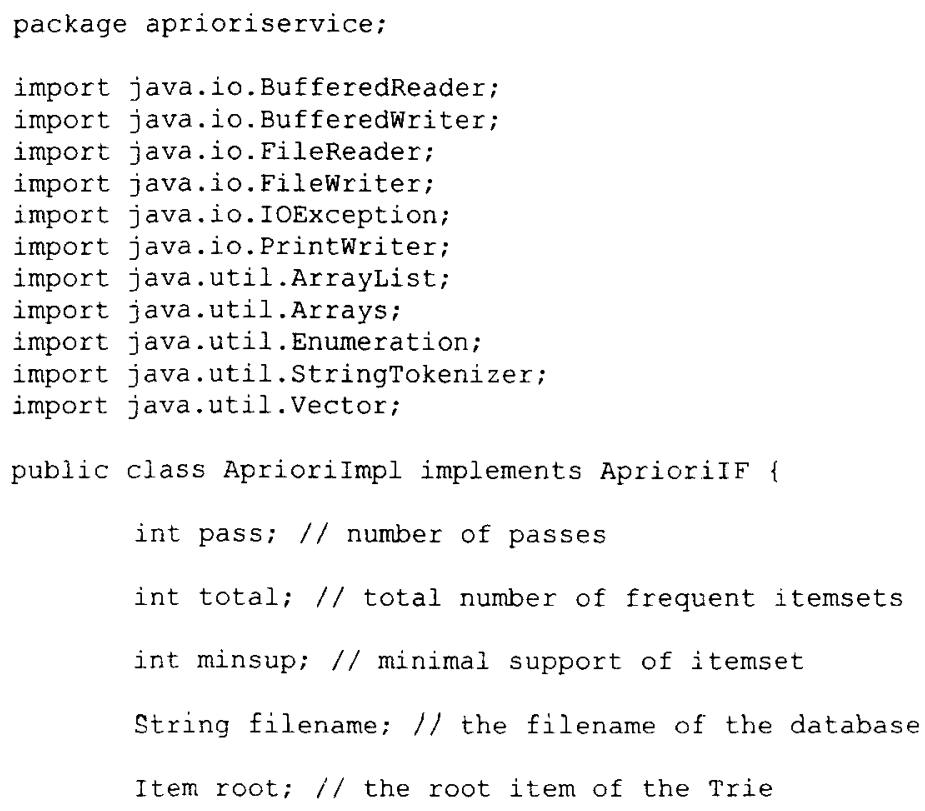


Bufferedwriter writer:// the buffer to write the output to

DataHandler dh; // the handler for the database

StringBuffer returnstr; // the buffer that holds all the messages

String outputfilename;

$1 * *$

* Constructur for creating a Apriori object.

*

$\star /$

public Apriorimpl() \{\}

$1 * *$

* The workhorse method for the basic implementation of the Apriori

* algorithm.

* $/$

public void findFrequentsets (String filename, String minsup, string outfile) f

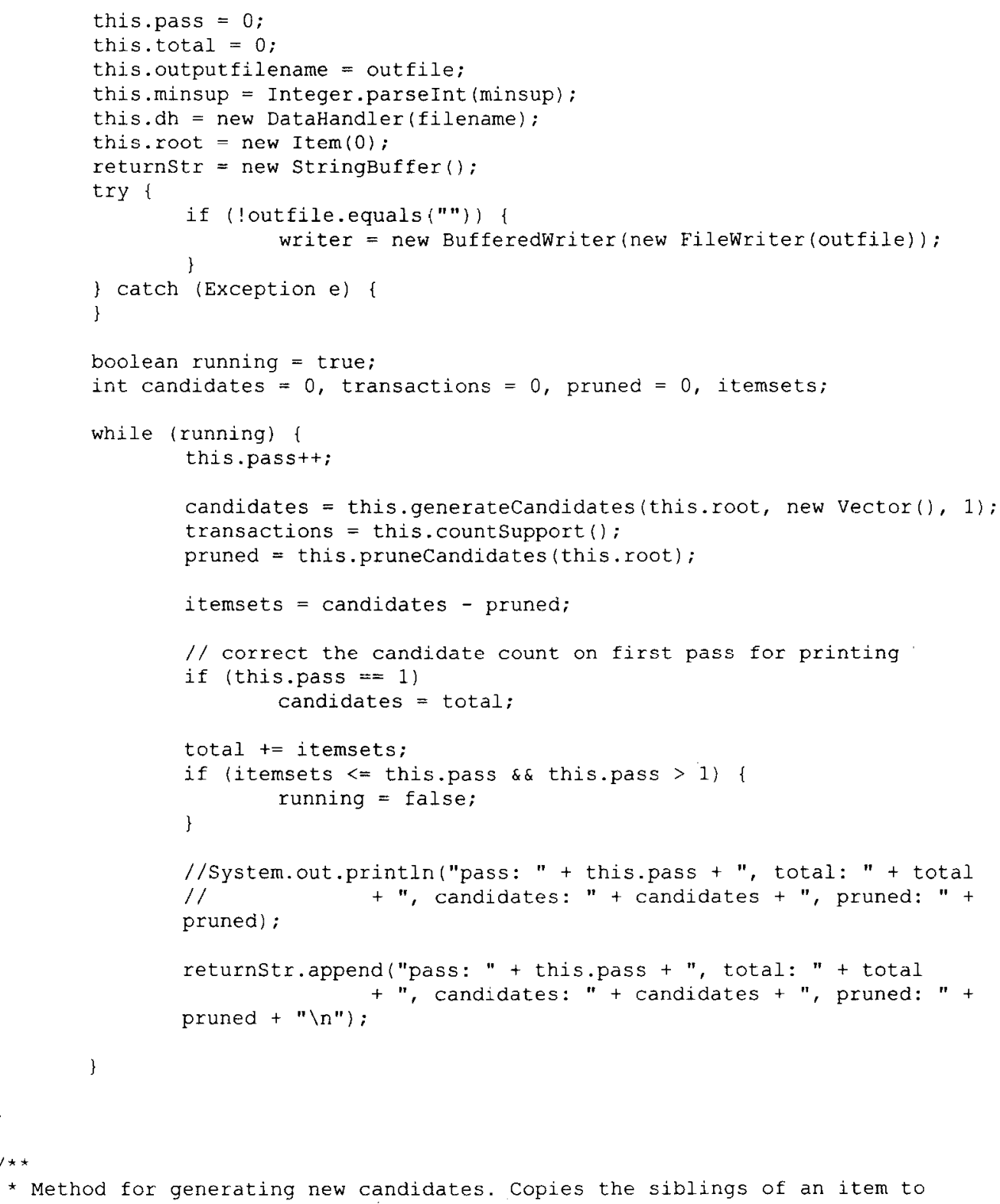




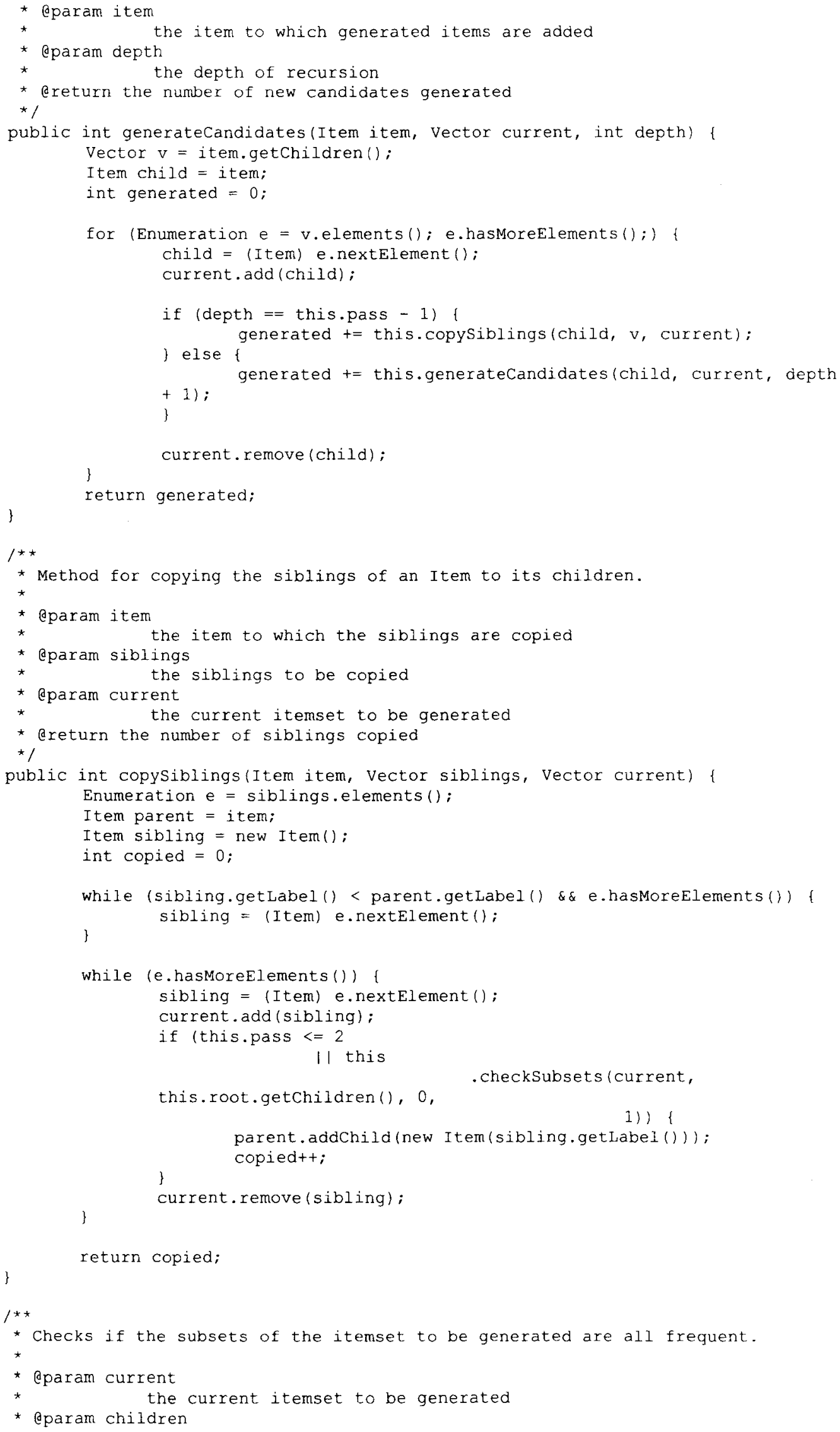




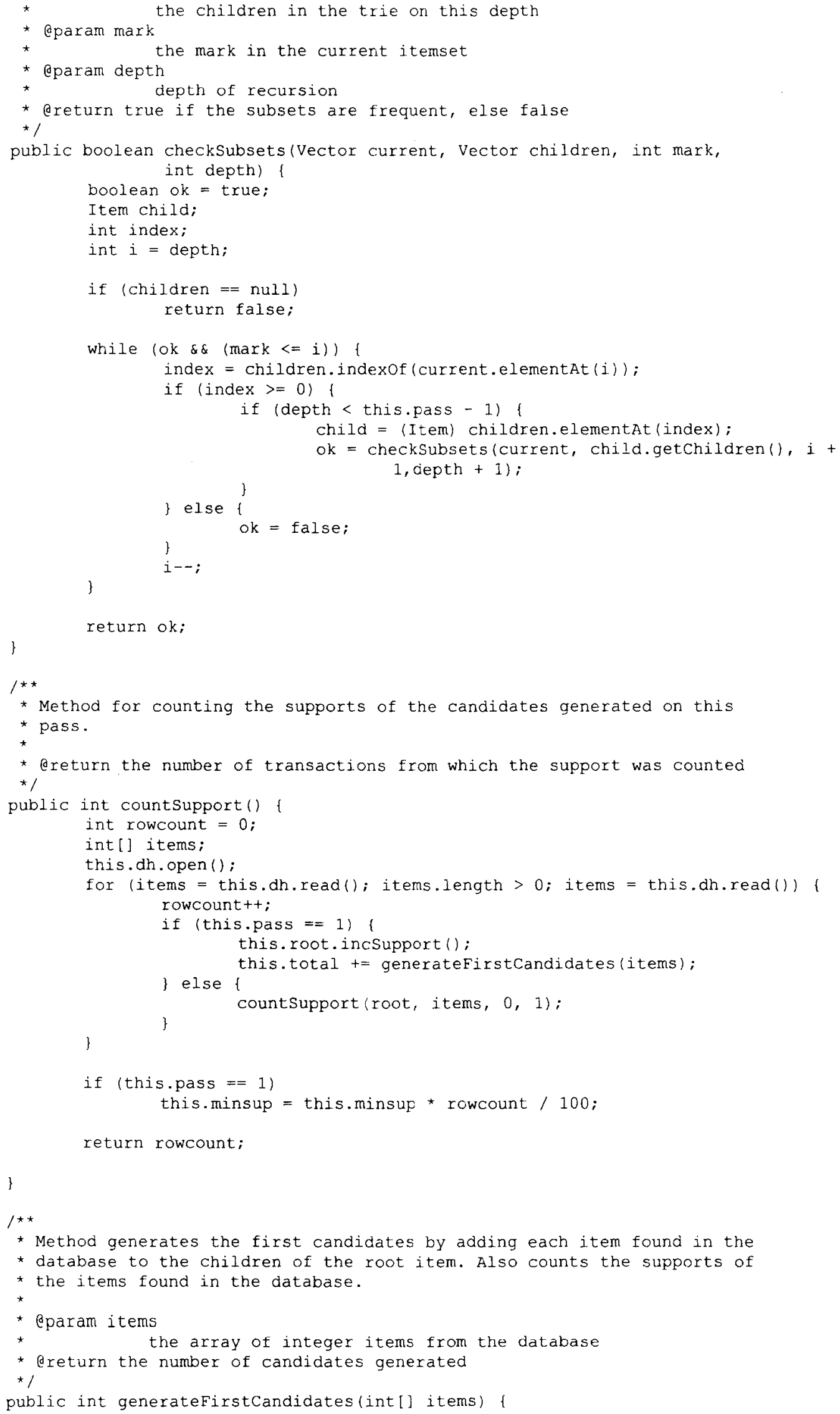




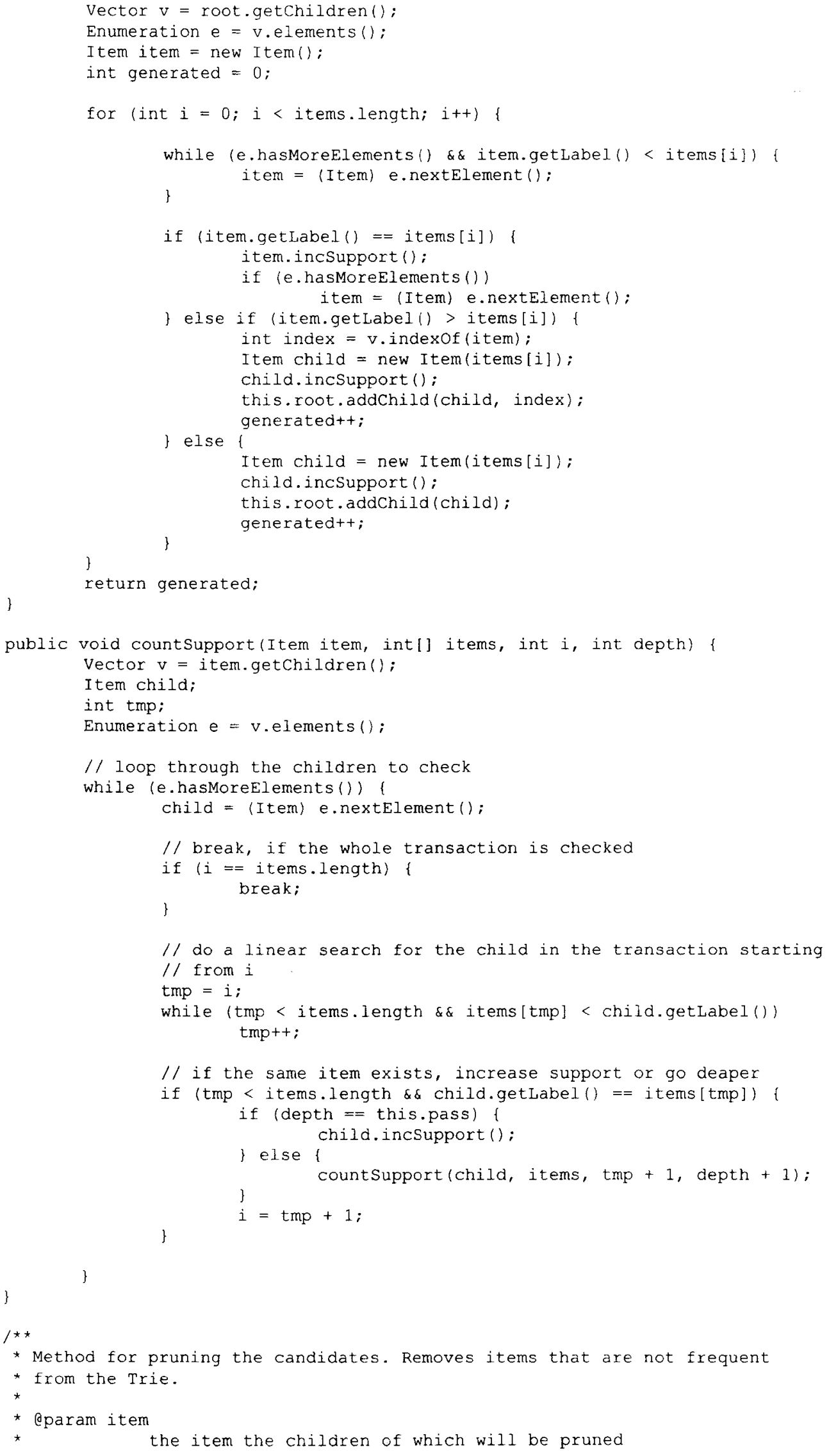




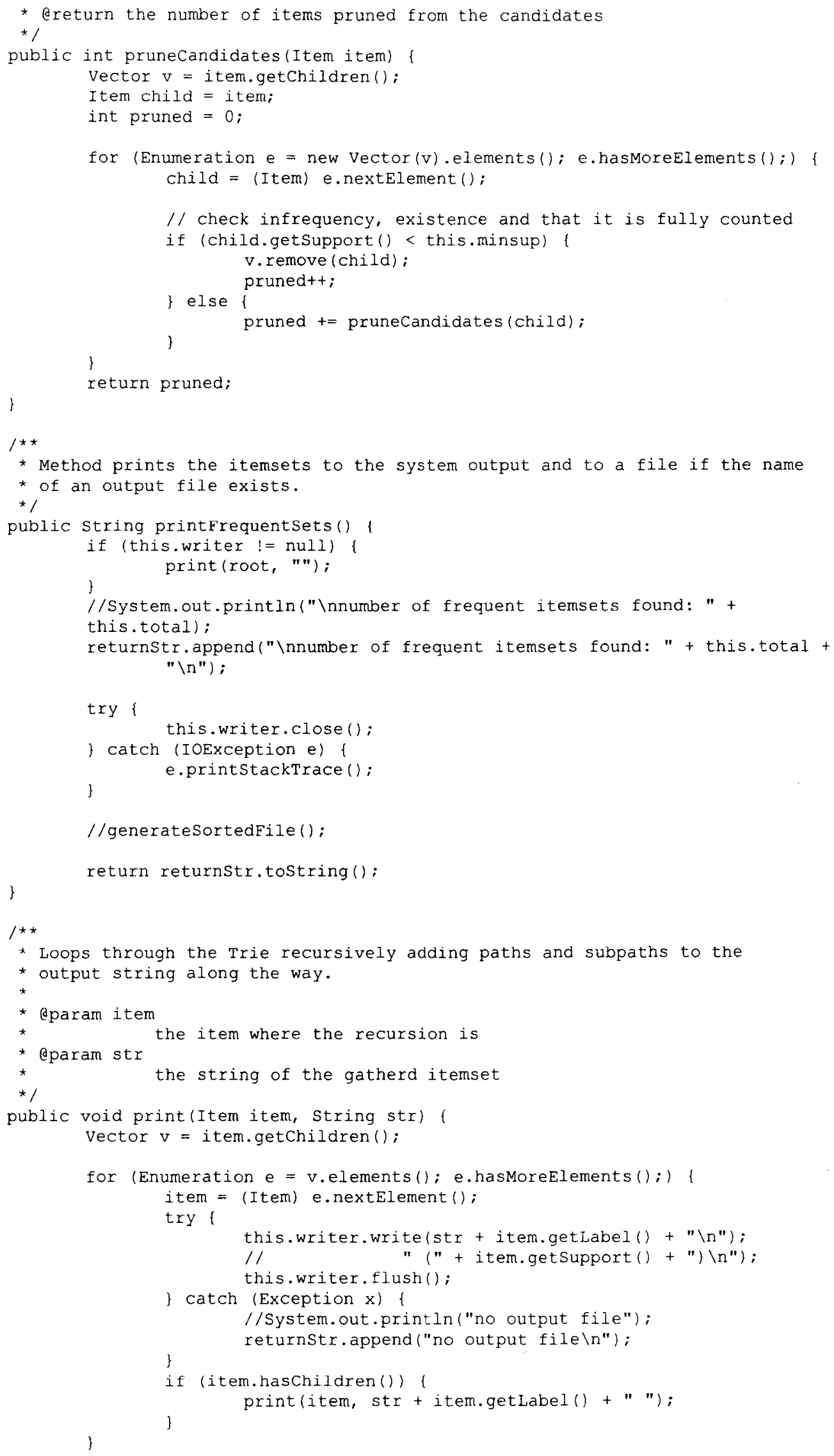


public void generateSortedFile(String outputfilename) i

try $i$

//Read the file contents into an arraylist

Arraylist al $=$ new Arraylist $(0)$;

BufferedReader in = new BufferedReader (new

FileReader (outputfilename));

String responseline $=$ null;

while ((responseline=in. readLine())!=null) (

Stringtokenizer st = new StringTokenizer(responseline);

//System.out.println(responseline + ":" +

st. countTokens ())

al.add (String.valueof (st.countrokens()) + ":" +

responseline);

)

in.close ();

/ Sort the elements

Object [] arrayForsorting = al.toArray();

Arrays. sort (arrayForSorting);

//System.out.println("Elements : " + arrayForSorting.length); Object [] invertArray = new Object [arrayForSorting.length];

for (int forward $=0$, reverse = arrayForsorting. length-1; forward< arrayforsorting.length; forward++, reverse--) 1

//System,out.println(" $i="$ + forward $+": "+j="+$ reverse);

invertArray[reverse] = arrayForSorting [forward];

/ Write to a new file

PrintWriter out $=$ new PrintWriter(new BufferedWriter (new Filewriter(outputfilename + "_sorted", false)));

for (int $j=0 ; j$ <arrayForSorting.length; $j++$ ) \{

StringTokenizer st $=$ new

stringTokenizer(invertarray [j].tostring(), ":"); st.nextToken();

out.printin(st.nextToken()):

out.close():

J catch (Exception e) (

// TODO Auto-generated catch block

e.printstackTrace();

\}

)

$1 * \star$

* Main method for testing the algorithm.

* Qparam args

* the arguments can contain the filename of the testfile and the

$\star$

minimal support threshold and a filename for output

$\star 1$

public static void main(String args[]) i

String testfile = "test.dat";

String outfile = "";

String support $=" 50 "$;

//Stopwatch $\mathrm{sw}=$ new Stopwatch();

//sw.start();

AprioriImpl apriori = new AprioriImpl();

apriori.findFrequentsets (testfile, support, outfile);

System.out.print In (apriori.printerequentsets ());

//sw.stop();

//sw.print (); 
FractalDimensionImpl.java - Implementation of the Fractal Dimension algorithm as a web service.

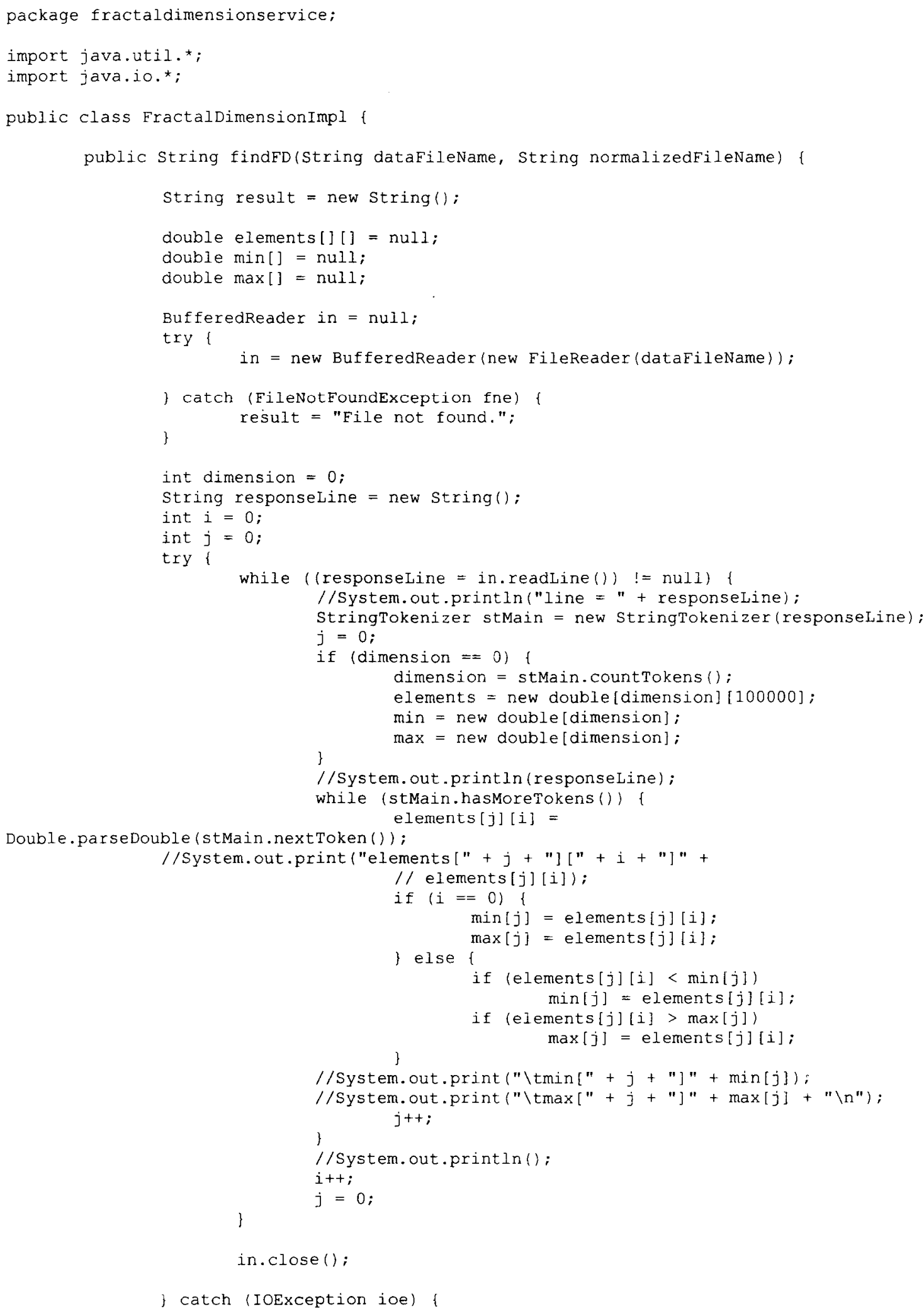




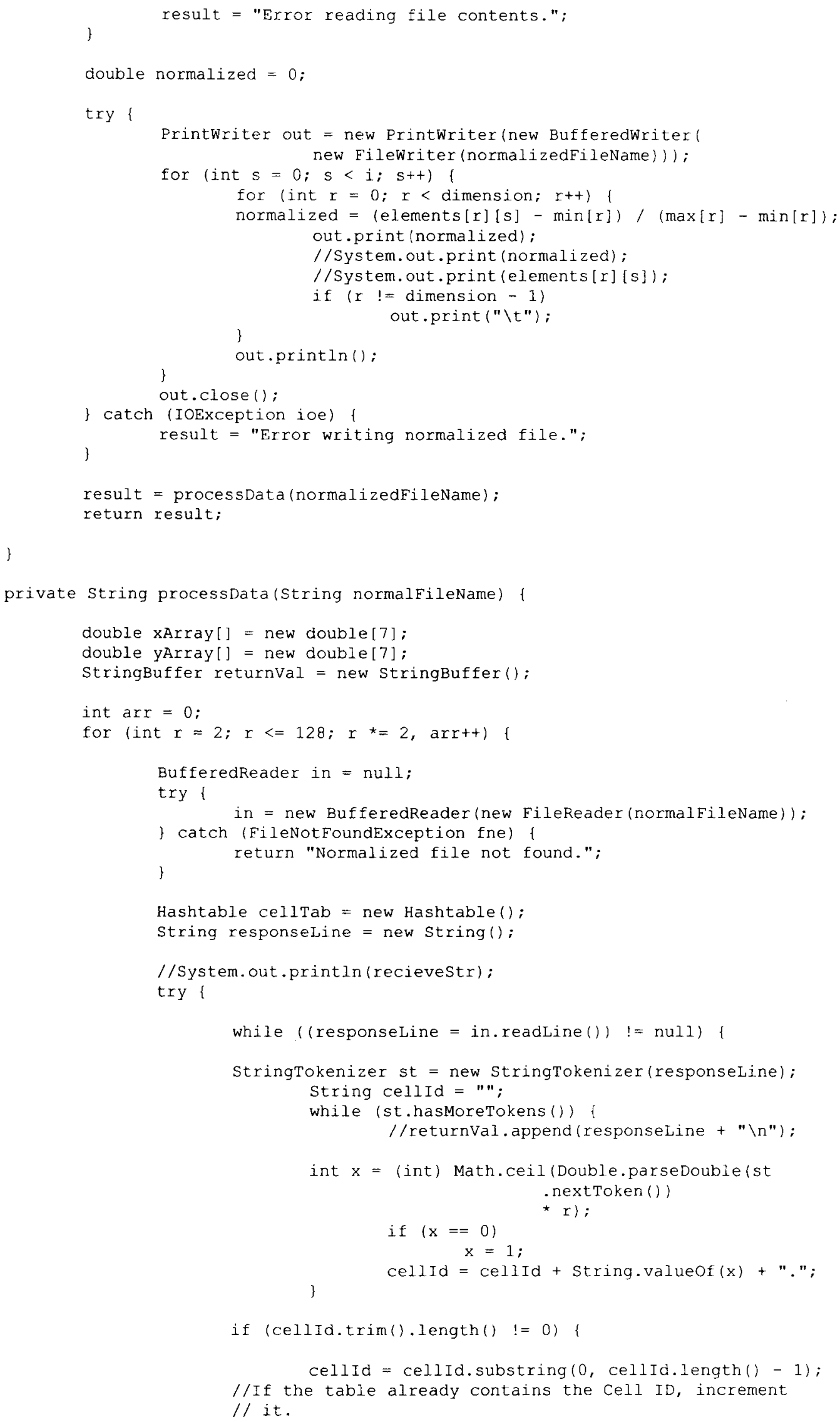




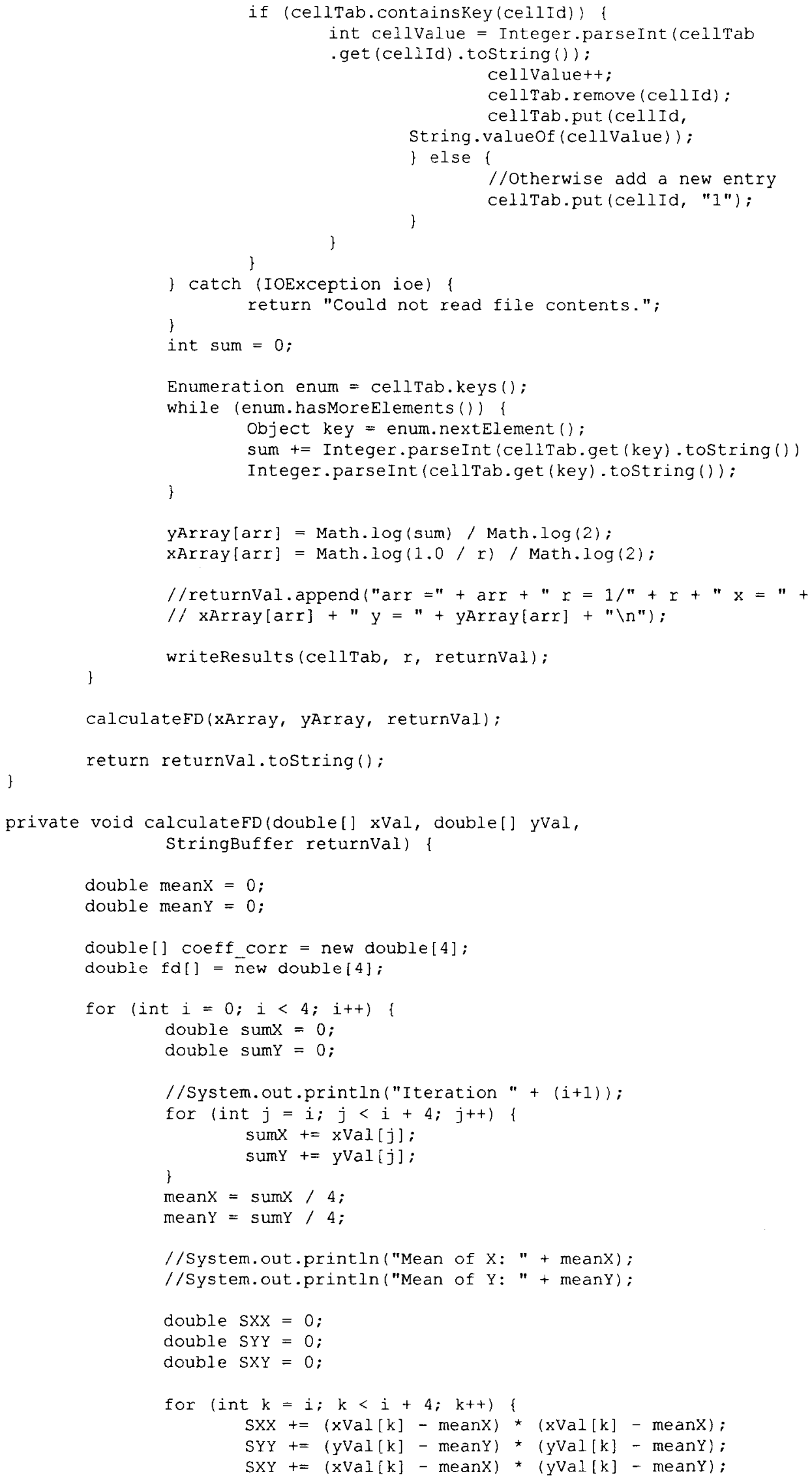




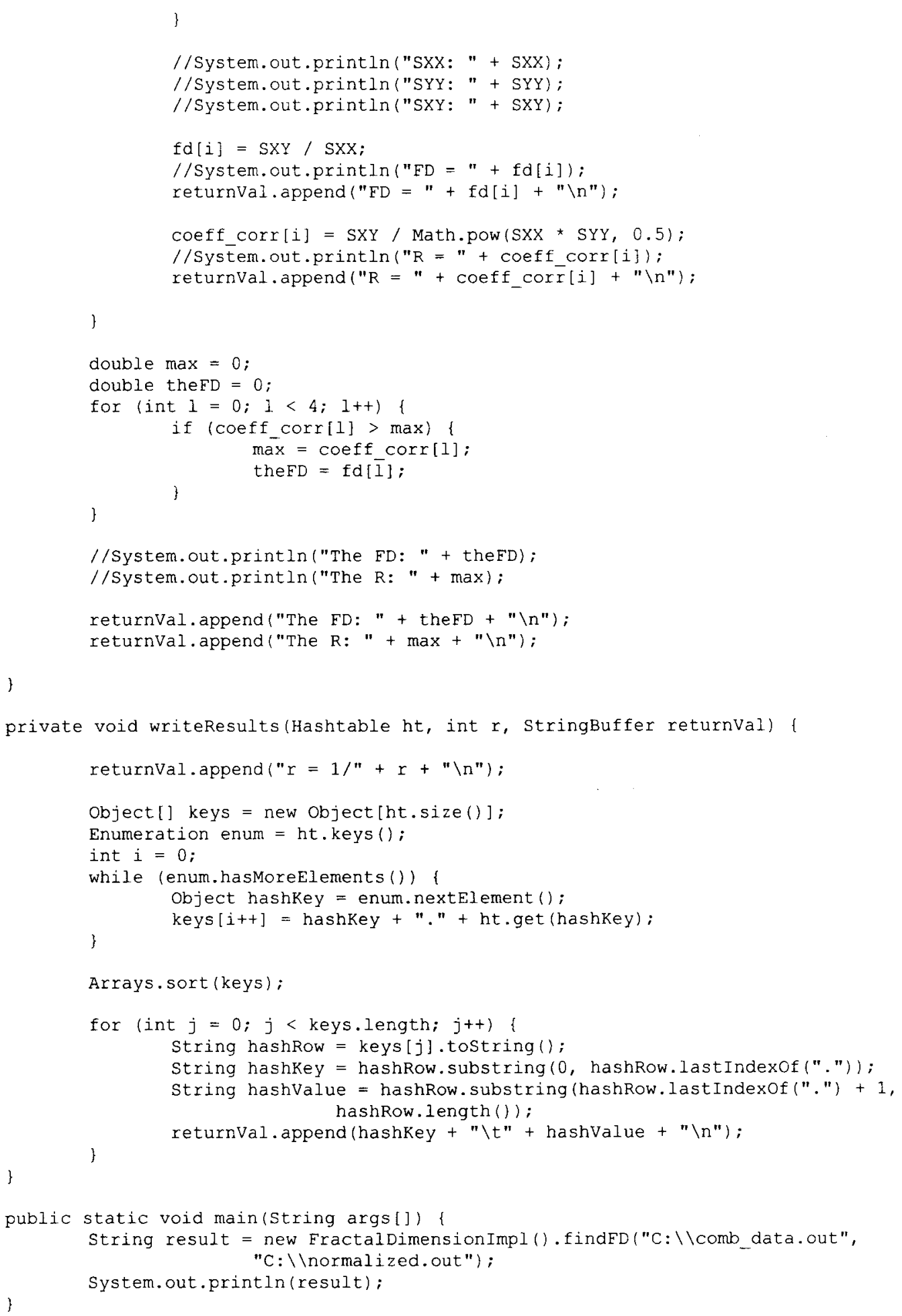




\section{CURRICULUM VitAE}

Name

Address

Date of Birth

Education

Publications
Padmanabhan Ramaswamy

Department of Computer Engineering and Computer Science University of Louisville

Louisville KY 40208

August 51976

M.S., Computer Engineering and Computer Science, 2002 - 2005 University of Louisville, Louisville, KY

Kerala University, Trivandrum, India B.S., Computer Science, 1994 - 1998

"An Extensible Service Oriented Distributed Data Mining Framework", Proceedings of International Conference on Machine Learning and Applications ICMLA - 04. 